\title{
Optical colours and spectral indices of $z=0.1$ EAGLE galaxies with the 3D dust radiative transfer code SKIRT
}

\author{
James W. Trayford, ${ }^{1 \star}$ Peter Camps, ${ }^{2}$ Tom Theuns, ${ }^{1}$ Maarten Baes, ${ }^{2}$ \\ Richard G. Bower, ${ }^{1}$ Robert A. Crain, ${ }^{3}$ Madusha L. P. Gunawardhana, ${ }^{4,1}$ \\ Matthieu Schaller, ${ }^{1}$ Joop Schaye ${ }^{5}$ and Carlos S. Frenk ${ }^{1}$ \\ ${ }^{1}$ Institute for Computational Cosmology, Durham University, South Road, Durham DH1 3LE, UK \\ ${ }^{2}$ Sterrenkundig Observatorium, Universiteit Gent, Krijgslaan 281, B-9000 Gent, Belgium \\ ${ }^{3}$ Astrophysics Research Institute, Liverpool John Moores University, 146 Brownlow Hill, Liverpool L3 5RF, UK \\ ${ }^{4}$ Instituto de Astrofísica and Centro de Astroingeniería, Facultad de Física, Pontificia Universidad Católica de Chile, Vicuña Mackenna 4860,7820436 \\ Macul, Santiago, Chile \\ ${ }^{5}$ Leiden Observatory, Leiden University, PO Box 9513, NL-2300 RA Leiden, the Netherlands
}

Accepted 2017 April 28. Received 2017 April 18; in original form 2016 October 20

\begin{abstract}
We present mock optical images, broad-band and $\mathrm{H} \alpha$ fluxes, and D4000 spectral indices for 30145 galaxies from the EAGLE hydrodynamical simulation at redshift $z=0.1$, modelling dust with the SKIRT Monte Carlo radiative transfer code. The modelling includes a subgrid prescription for dusty star-forming regions, with both the subgrid obscuration of these regions and the fraction of metals in diffuse interstellar dust calibrated against far-infrared fluxes of local galaxies. The predicted optical colours as a function of stellar mass agree well with observation, with the SKIRT model showing marked improvement over a simple dust-screen model. The orientation dependence of attenuation is weaker than observed because EAGLE galaxies are generally puffier than real galaxies, due to the pressure floor imposed on the interstellar medium (ISM). The mock $\mathrm{H} \alpha$ luminosity function agrees reasonably well with the data, and we quantify the extent to which dust obscuration affects observed $\mathrm{H} \alpha$ fluxes. The distribution of D4000 break values is bimodal, as observed. In the simulation, 20 per cent of galaxies deemed 'passive' for the SKIRT model, i.e. exhibiting D4000 >1.8, are classified 'active' when ISM dust attenuation is not included. The fraction of galaxies with stellar mass greater than $10^{10} \mathrm{M}_{\odot}$ that are deemed passive is slightly smaller than observed, which is due to low levels of residual star formation in these simulated galaxies. Colour images, fluxes and spectra of EAGLE galaxies are to be made available through the public EAGLE data base.
\end{abstract}

Key words: dust, extinction-galaxies: star formation.

\section{INTRODUCTION}

Cosmological simulations are instrumental for our understanding of how competing physical processes shape galaxies. $\mathrm{N}$-body simulations played a crucial role in establishing the cold dark matter (CDM) paradigm, demonstrating that dark matter haloes provide the potential wells into which gas can collapse, cool and form stars (e.g. White \& Rees 1978; Frenk et al. 1988; White \& Frenk 1991). Simulations that also include hydrodynamics have by now matured to such an extent that they show good agreement between simulated and observed galaxies for a wide range of properties, provided feedback from forming stars and accreting black holes is implemented to be very efficient (e.g. Vogelsberger et al. 2014; Murante et al. 2015; Schaye et al. 2015; Davé, Thompson \& Hopkins 2016). Differences in the properties of simulated galaxies result primarily from the choices made in how to implement unresolved physical processes, particularly star formation and feedback, as shown by e.g. Schaye et al. (2010), Scannapieco et al. (2012) and Kim et al. (2014).

The comparisons to data are relatively indirect, however, because simulations yield intrinsic properties of galaxies, such as galaxy stellar mass or star formation rate (SFR), that are not directly observable. 'Inverse models' attempt to infer such physical properties from the observed fluxes. The main ingredients of such models are the assumed stellar initial mass function (IMF), templates for the star formation and enrichment histories, a model for dust effects (absorption and scattering), and a model for stellar evolution 
encapsulated by a population synthesis description to yield fluxes. This is exemplified by the analysis of Li \& White (2009) applied to the 7th data release (DR7; Abazajian et al. 2009) of the Sloan Digital Sky Survey (SDSS, York et al. 2000), or the analysis by Baldry et al. (2007) applied to the Galaxy And Mass Assembly (GAMA, Driver et al. 2009) survey. Such analysis makes necessarily bold simplifications, for example assuming exponential star formation histories, uniform stellar metallicities and a dust-screen model. Mitchell et al. (2013) demonstrated how this methodology suffers from degeneracy between the star formation history, metallicity and dust properties of galaxies.

Similar models are needed to infer SFRs or passive fractions. The strength of the $\mathrm{H} \alpha$ recombination line is sensitive to recent star formation, as it probes UV-continuum emission from stars that are $\lesssim 10$ Myr old (Kennicutt 1998). However, a significant fraction of the $\mathrm{H} \alpha$ flux in star-forming galaxies is emitted by dusty $\mathrm{H}$ II regions (e.g. Zurita, Rozas \& Beckman 2000), and therefore the conversion from flux to SFR requires a model to account for obscuration (e.g. James et al. 2004; Best et al. 2013; Gunawardhana et al. 2013). Similarly, the continuum strength on either side of the $4000 \AA$ break depends on the relative contribution to the flux of old versus younger stars, and hence is a useful proxy for the specific SFR of a galaxy (e.g. Balogh et al. 1999; Kauffmann et al. 2003a). However, the amplitude of the break may also be affected by dust, and hence the observed passive fraction depends on the assumed dust properties.

In addition to inverse modelling, it should be possible to apply the ingredients of the inverse models to the simulated galaxies instead, and compare mock fluxes to the observations. Such 'forward models' have many potential advantages. For example, the star formation histories of simulated galaxies are more detailed and diverse than the parametric models used in inverse modelling. Similarly, the simulated - and presumably also the observed stars in any galaxy - have a considerable spread in metallicity, rather than a single uniform value. These assumed priors may introduce biases in the inferred properties of galaxies, see e.g. Trayford et al. (2016). Notwithstanding any practical considerations, surely it should be the ultimate aim of the simulations to predict observables.

In practice there are still formidable challenges with forward modelling, and inverse and forward modelling approaches are largely complementary. Inverse modelling is useful to assess how distinct physical quantities may contribute to observables and elucidate discrepancies between real and mock observations, while insights gained from a forward modelling approach can inform and improve our inverse models. For instance, generating mock galaxy observations with attenuation and re-emission by dust can demonstrate how numerous degeneracies in spectral energy distribution (SED) inversion can be lifted by incorporating far-infrared (FIR) observations (e.g. Hayward \& Smith 2015). The treatment of dust, however, exemplifies a major challenge of forward modelling. While dust represents a marginal fraction of the mass in galaxies and simulations do not typically model an explicit dust phase (with exceptions, e.g. Bekki 2015; Aoyama et al. 2017; McKinnon et al. 2017), interstellar dust can play an important role in processing the light we observe from galaxies.

On average, about a third of the UV plus optical starlight emitted in local star-forming galaxies is absorbed by dust and re-radiated at longer wavelengths (e.g. Popescu \& Tuffs 2002; Viaene et al. 2016). The effect of dust attenuation introduces various systematics, particularly for young stellar populations: the cross-sections for absorption and scattering generally increase with the frequency of incident light, such that the relatively blue emission from young stars is more affected. But the impact of dust also depends on the morphology and orientation of the galaxy: young stellar populations are typically found in a thin disc and near the dense, dusty ISM regions from which they formed. These regions are therefore likely to have relatively high dust obscuration, and, if this is not accounted for, may affect inferred structural measures of galaxies such as scalelengths/scaleheights and bulge-to-disc ratios. Because young stars may be more obscured than old stars, the attenuation curve (the ratio of observed overemitted radiation as function of wavelength for the observed galaxy) of a galaxy differs in general from the extinction curve that describes the wavelength dependence of the photon-dust interaction. The modelling of dust is clearly an important aspect of comparing models to observations, and is the subject of this paper.

An idealized dust model is that of an intervening dust screen with a wavelength-dependent optical depth, the attenuation of which can be computed analytically. The geometry of the dust distribution and some effects of scattering, can be accounted for to some extent by making the attenuation curve 'greyer' (i.e. less wavelength dependent) than the extinction curve (e.g. Calzetti 2001), and/or by using multiple screen components (e.g. Charlot \& Fall 2000). Trayford et al. (2015, hereafter T15) adopted the two component screen model of Charlot \& Fall (2000) to represent dust absorption in the EAGLE simulations (Schaye et al. 2015) when generating broad-band luminosities and colours. Absorption is boosted by a fixed factor for young ( $<30 \mathrm{Myr}$ ) stars in this model, and the overall optical depths depend on the gas properties of each simulated galaxy with additional scatter to account for orientation dependence.

T15 showed that optical colours and luminosities generated for EAGLE galaxies are broadly compatible with the GAMA measurements, while exhibiting some notable discrepancies. In particular, the modelling resulted in a more pronounced bimodal distribution of colours at a given stellar mass than observed. In particular, model colours exhibited bimodality amongst even the most massive galaxies for which bimodality is absent in the data. A related discrepancy was that the red and blue fractions were also somewhat inconsistent between model and data, with the model yielding an excess of blue galaxies at high $M_{\star}$. The cause of these discrepancies was attributed to both differences between the intrinsic properties of the simulated and observed galaxy populations (higher specific SFRs in EAGLE), as well as uncertainties in the modelling of the photometry (especially dust effects). Of particular concern was that lower than observed average SFRs in EAGLE galaxies (Furlong et al. 2015) and underestimated reddening could have a compensatory effect, potentially yielding a fortuitous agreement with GAMA colours.

The dust-screen model applied by T15 may not represent attenuation in EAGLE galaxies well. Indeed, the effects of complex geometries cannot be captured by a screen (e.g. Witt, Thronson \& Capuano 1992): there are degeneracies between dust geometry and dust content. Colours of galaxies where dust and stars are well mixed can be confused with dimmer dust-free galaxies if a screen is assumed (e.g. Disney, Davies \& Phillipps 1989; Calzetti 2001). Screens also neglect scattering into the line of sight, or attempt to account for it with approximative absorption. With scattering being as important as geometry at optical wavelengths, and often producing effects entirely dissimilar to absorption, a screenbased approximation is often insufficient (Byun, Freeman \& Kylafis 1994; Baes \& Dejonghe 2001).

Fully accounting for dust requires three-dimensional radiative transfer calculations (e.g. Steinacker, Baes \& Gordon 2013), and, given the lack of symmetry, Monte Carlo radiative transfer (MCRT) (e.g. Whitney 2011) techniques appear to be well suited. These follow the path of photons from sources through the dusty ISM 
to a camera. A variety of MCRT codes are publicly available, such as SUNRISE (Jonsson 2006) and SKIRT (Baes et al. 2003, 2011; Camps \& Baes 2015). sunRISE has been applied to zoomed galaxy simulations (e.g. Jonsson, Groves \& Cox 2010; Guidi, Scannapieco $\&$ Walcher 2015), and by Torrey et al. (2015) to compute images of galaxies from the ILLUSTRIs simulation (Vogelsberger et al. 2014). Note, however, that Torrey et al. (2015) did not actually include dust in the SUNRISE models. SKIRT has been applied to galaxy models (e.g. Gadotti, Baes \& Falony 2010; Saftly et al. 2015) but also to dust around active galactic nuclei (AGNs) (Stalevski et al. 2012, 2016). Full MCRT dust models of simulated galaxies in a cosmologically representative volume are yet to be published.

Previous studies that apply MCRT dust modelling to galaxy zoom simulations can provide insight into this approach. Scannapieco et al. (2010) use MCRT to produce representative optical images and decompose them into bulge and disc contributions, but find that dust effects are negligible due to low gas fractions and metallicities in the simulations themselves. Simulations with more realistic gas phase metallicities have also been processed with MCRT to produce mock observables across the UV to sub-mm wavelength range (e.g. Jonsson et al. 2010; Guidi, Scannapieco \& Walcher 2015; Hayward $\&$ Smith 2015). The influence of galaxy orientation and geometry on attenuation properties and recovered physical quantities are explored by Hayward \& Smith (2015), showing how the effective attenuation curves vary with orientation and morphological transformation for idealized merger zooms.

In this paper, we generate optical broad-band fluxes and spectra for EAGLE galaxies using SKIRT, comparing mock fluxes to GAMA observations and to the dust-screen model of T15. EAGLE (Crain et al. 2015; Schaye et al. 2015) is a suite of hydrodynamical SPH (smoothed particle hydrodynamics)-simulations, with subgrid parameters calibrated to a small set of low-redshift $(z \sim 0.1)$ observables. The simulation reproduces a variety of observations that were not part of the calibration procedure, such as the neutral and molecular contents of $z \sim 0$ galaxies (Lagos et al. 2015; Bahé et al. 2016), and the evolution of galaxy SFRs and sizes (Furlong et al. 2015, 2017). There is a hint that the simulation underpredicts specific SFRs except for the most massive galaxies.

The simulation does not trace dust explicitly: we describe dust associated with star-forming regions using the MAPPINGS models by Groves et al. (2008), and assume that the ISM dust/gas ratio depends on metallicity. This procedure was developed for this work and for the companion paper of Camps et al. (2016), who looked at the FIR properties of present-day EAGLE galaxies. This paper compared SKIRT models to FIR observations of local galaxies to calibrate dust models, showing that observed dust scaling relations can be reproduced. Camps et al. (2016) uses dust parameters identical to those used in the present work. The influence of these parameters is discussed in Section 3 and the Appendix.

The paper is organized as follows: Section 2 provides a summary of the EAGLE simulations used in this work, how we define galaxies in our simulated sample and the data sets we compare to. Section 3 details the procedure used to produce observables with SKIRT. We investigate the predicted photometric colours in Section 5 and compare the effects of dust to the screen model approach of T15. In Section 6, we present novel measurements of spectral indices for EAGLE galaxies, and again quantify dust effects. We focus in particular on the star formation proxies of $\mathrm{H} \alpha$ and the extent to which EAGLE reproduces the statistics of, and the correlation in, the D4000 break. We summarize our findings and conclude in Section 7. Those only concerned with our main results may want to read from Section 5 onwards; outputs of the model are described in Section 3.3.
Table 1. Numerical parameters of those simulations of the EAGLE suite that are used in this paper. From left to right: simulation identifier, side length of cubic volume $L$ in comoving Mpc (cMpc), initial mass $m_{\mathrm{g}}$ of baryonic particles, Plummer equivalent gravitational softening $\epsilon_{\text {prop }}$ scale at redshift $z=0$ in pkpc, where we use 'pkpc' to denote proper kiloparsecs.

\begin{tabular}{lccc}
\hline Name & $\begin{array}{c}L \\
(\mathrm{cMpc})\end{array}$ & $\begin{array}{c}m_{\mathrm{g}} \\
\left(\mathrm{M}_{\odot}\right)\end{array}$ & $\begin{array}{c}\epsilon_{\mathrm{prop}} \\
(\mathrm{pkpc})\end{array}$ \\
\hline Ref-L025N0376 (Ref-25) & 25 & $1.81 \times 10^{6}$ & 0.70 \\
Ref-L025N0754 (RefHi-25) & 25 & $2.26 \times 10^{6}$ & 0.35 \\
Recal-L025N0754 (Recal-25) & 25 & $2.26 \times 10^{5}$ & 0.35 \\
Ref-L100N1504 (Ref-100) & 100 & $1.81 \times 10^{6}$ & 0.70 \\
\hline
\end{tabular}

Table 2. Properties of galaxies shown in Fig. 2. All properties are extracted from the EAGLE data base (McAlpine et al. 2016), for a $30 \mathrm{pkpc}$ aperture.

\begin{tabular}{lllc}
\hline Type & Galaxy ID & $M_{\star}\left(\mathrm{M}_{\odot}\right)$ & $\mathrm{SFR}\left(\mathrm{M}_{\odot} \mathrm{yr}^{-1}\right)$ \\
\hline Late (S) & 15814162 & $9.94 \times 10^{10}$ & 3.96 \\
Irregular (Irr) & 14318126 & $1.34 \times 10^{11}$ & 4.60 \\
Early (E) & 19099219 & $2.05 \times 10^{10}$ & 0.00 \\
\hline
\end{tabular}

The mock EAGLE observables used in this work, and additional data products listed in Section 3.3, are to be made available via the public data base (McAlpine et al. 2016). The modelling, described and tested at low redshift $(z \leq 0.1)$, is also used to provide these mock observables for galaxies taken from EAGLE simulations and redshifts that are not considered in this work.

\section{SIMULATIONS AND DATA}

We provide a brief overview of the EAGLE simulation suite, see Schaye et al. (2015), Crain et al. (2015), hereafter S15 and C15, respectively, for full details, review the population synthesis model and dust treatment of T15, and briefly describe the volume-limited sample of galaxies compiled from the GAMA survey (Driver et al. 2009).

\subsection{The EAGLE simulation suite}

EAGLE comprises a suite of cosmological hydrodynamical simulations of periodic cubic volumes performed using a modified version of the GADGET-3 TREESPH code (which is an update of the GADGET2 code last described by Springel, Di Matteo \& Hernquist 2005). Simulations were performed for a range of volumes and numerical resolutions. Here we concentrate on the 'reference' model, using simulations at different resolution to assess numerical convergence. In particular, we use models L100N1504, L025N0376 and L025N0752 from Table 2, and Recal from table 3 of S15; in our Table 1 we refer to these simulations as Ref-100, Ref25, RefHi-25 and Recal-25, respectively. The EAGLE suite assumes a $\Lambda \mathrm{CDM}$ cosmology with parameters derived from the initial Planck (Planck Collaboration et al. 2014) satellite data release $\left(\Omega_{\mathrm{b}}=0.0482, \Omega_{\text {dark }}=0.2588, \Omega_{\Lambda}=0.693\right.$ and $h=0.6777$, where $H_{0}=100 \mathrm{~h} \mathrm{~km} \mathrm{~s}^{-1} \mathrm{Mpc}^{-1}$ ). Some simulation details are listed in Table 1.

We focus primarily on the Ref-100 simulation volume. The $100^{3} \mathrm{Mpc}^{3}$ volume and mass resolution of $1.2 \times 10^{6} \mathrm{M}_{\odot}$ in gas for Ref-100 provides a sample of $\sim 30000$ galaxies resolved by $>1000$ star particles at redshift $z=0.1$, with $\sim 3000$ galaxies resolved by $>10000$ star particles. In addition to this primary sample of galaxies, we also use the higher resolution Ref-25 and Recal-25 
simulations to test the 'strong' and 'weak' convergence (see S15 for definition of these terms). The $25^{3} \mathrm{Mpc}^{3}$ volumes have a factor $2\left(2^{3}\right)$ superior spatial (mass) resolution than Ref-100. As Ref-25 uses the same fiducial model at high resolution (with the same initial phases and amplitudes of the Gaussian field), it may be used to test the strong convergence of galaxy properties. The feedback efficiencies adopted by Recal-25 were recalibrated to provide better agreement with the $z=0.1$ galaxy stellar mass function at high resolution and to test the weak convergence (see also $\mathrm{C} 15$ ).

The initial conditions of all EAGLE simulations were generated appropriately for a starting redshift of $z=127$ using an initial perturbation field generated with the PANPHASIA code described by Jenkins \& Booth (2013). SPH is implemented as in Springel et al. (2005), but using the pressure-entropy formulation of Hopkins (2013), including artificial conduction and viscosity (Dehnen \& Aly 2012), a time-step limiter (Durier \& Dalla Vecchia 2012) and the C2 kernel of Wendland (1995). These modifications to the standard GADGET-3 implementation are collectively termed as ANARCHY (Dalla-Vecchia in preparation, summarized in appendix A of S15). Schaller et al. (2015) show that these ANARCHY modifications are important in the largest EAGLE haloes, but have minimal effect on galaxies of stellar mass $M_{\star} \lesssim 10^{11} \mathrm{M}_{\odot}$. To represent important astrophysical process acting on scales below the resolution of EAGLE, a number of subgrid modules are also employed in the code. Relevant modules include schemes for star formation, enrichment and mass loss by stars, photoheating, radiative cooling and thermal feedback associated with accreting black holes and the formation of stars, as described below.

Star formation is treated stochastically in EAGLE. SFRs are calculated for individual gas particles using a pressure-dependent formulation of the empirical Kennicutt-Schmidt law (Schaye \& Dalla Vecchia 2008), with a metallicity-dependent density threshold below which SFRs are zero (Schaye 2004). Gas particles thus may have some probability of being wholly converted into a star particle at each time step, inheriting the initial element abundances of their parent particle. The gravitational softening scales listed in Table 1 provide a practical limit on spatial resolution. Cold, dense gas $\left(T<10^{4} \mathrm{~K}, n_{\mathrm{H}}>0.1 \mathrm{~cm}^{-3}\right)$ with Jeans lengths below these scales is thus unresolved, and any corresponding gas would artificially fragment in the simulation. To ensure that the Jeans mass of gas is always resolved (albeit marginally), a pressure floor is enforced via a single-phase polytropic equation of state, $P_{\mathrm{EoS}} \propto \rho^{\gamma_{\mathrm{EoS}}}$. Once formed, star particles are treated as simple stellar populations (SSPs), assuming a universal Chabrier (2003) stellar IMF. These SSPs lose mass and enrich neighbouring gas particles according to the prescription of Wiersma et al. (2009b), accounting for type Ia and type II supernovae and winds from massive and AGB stars. Eleven individual elements $(\mathrm{H}, \mathrm{He}, \mathrm{C}, \mathrm{N}, \mathrm{O}, \mathrm{Ne}, \mathrm{Mg}, \mathrm{Si}, \mathrm{S}, \mathrm{Ca}$ and $\mathrm{Fe}$ ) are followed, as well as a 'total' metallicity (the mass fraction in elements more massive than $\mathrm{He}$ ), $Z$.

Two types of abundances are tracked for the gas in EAGLE, a particle abundance that is changed through direct enrichment by star particles and a smoothed abundance that smooths particle abundances between neighbours using the SPH kernel (see Wiersma et al. 2009b). Diffusion is not implemented in the simulation; therefore, no metals are exchanged between gas particles. This may occasionally lead to individual particles exhibiting extreme values as well as large variations in metallicity, even for close neighbours. Although the SPH smoothing is not strictly representative of metal diffusion, it does mitigate extreme values and reduces stochasticity in the metal distribution. For this reason we adopt the smoothed metallicities throughout this study, which were also used to compute cooling rates and nucleosynthetic yields during the simulation.

The energy that stellar populations inject into the interstellar medium (ISM) through supernovae, stellar winds and radiation is collectively termed stellar feedback. Stellar feedback is implemented per star particle (and is separate from enrichment) using the thermal feedback scheme described by Dalla Vecchia \& Schaye (2012). This implementation sets a temperature change $\Delta T_{\mathrm{SF}}$, the temperature by which stochastically sampled gas particle neighbours of stars are heated. The value of $\Delta T_{\mathrm{SF}}=10^{7.5} \mathrm{~K}$ is chosen for the reference model; this is high enough to mitigate catastrophic numerical losses, while low enough to prevent the probability of heating for neighbouring gas particles, $p_{\mathrm{SF}}$, from becoming small and leading to poor sampling (see $\mathrm{S} 15$ ). The $p_{\mathrm{SF}}$ value depends on both $\Delta T_{\mathrm{SF}}$ and the fraction of energy that couples to heat the ISM. The latter fraction is allowed to vary with local gas properties and is calibrated to reproduce observed local galaxy sizes, as detailed by $\mathrm{C} 15$.

Black holes are seeded in haloes with mass exceeding $10^{10} h^{-1} \mathrm{M}_{\odot}$, following Springel et al. (2005). The most bound gas particle is then converted to a black hole particle with a subgrid mass of $10^{5} h^{-1} \mathrm{M}_{\odot}$. The black hole grows by subgrid accretion as detailed in S15 and Rosas-Guevara et al. (2015). A fixed 1.5 per cent of the rest-mass energy in accreted material provides the energy budget for black hole feedback. This is implemented using a similar stochastic scheme as used for injecting stellar feedback, but with a higher heating temperature $\left(\Delta T_{\mathrm{BH}}=10^{8.5} \mathrm{~K}\right.$ for the reference models, and $10^{9} \mathrm{~K}$ for Recal-25).

Photoheating and radiative cooling are implemented as described by Wiersma, Schaye \& Smith (2009a), based on the 11 elements traced. This model assumes that gas is in photoionization equilibrium with the cosmic UV+X-ray background as calculated by Haardt \& Madau (2001).

We use the friends-of-friends algorithm with a linking length of 0.2 times the mean inter particle separation (Davis et al. 1985; Lacey \& Cole 1994) to identify haloes. Self-bound substructures within these haloes (subhaloes) are identified with the SUBFIND algorithm (Springel et al. 2001). Subhaloes may comprise dark matter, stars and gas. Each galaxy is associated with a separate subhalo.

\subsection{Population synthesis and analytic dust treatment}

(Trayford et al. 2015, T15) presented model photometry for EAGLE galaxies at $z=0.1$. The model adopted here is based on that implementation with some differences as described below. We begin with a brief overview of the T15 model. T15 calculated photometric fluxes in standard ugrizYJHK broad-bands (Hewett et al. 2006; Doi et al. 2010), which included an analytic model for dust obscuration. Stellar emission was calculated using the GALAXEV population synthesis model (Bruzual \& Charlot 2003). This parametrization uses the stellar ages, smoothed metallicities and initial masses described in Section 2.1. We adopt the same parametrization in this work where possible. T15 also 're-sampled' the young stellar component at increased mass resolution, to reduce artificial stochasticity in colours resulting from the coarse sampling of star formation in EAGLE. We use a similar approach here, with full details provided in Section 3.1.2. The fiducial dust model employed by T15 was based on the two component dust screen of Charlot \& Fall (2000), with the optical depth additionally depending on the gas properties and including a prescription to account for orientation-dependent dust obscuration. 
In this paper, we adopt many of the same surveying and modelling procedures: individual subhaloes are considered potential galaxy hosts, with the 'galaxy' comprising the bound material within a $30 \mathrm{pkpc}$ spherical aperture centred on the subhalo's baryonic centre of mass. This choice was initially made in S15 to reasonably approximate a Petrosian aperture, and we adopt this galaxy definition for consistency with prior measurements of various galaxy properties. In all but the most massive galaxies, the bound mass excluded by the aperture is negligible (see S15 for details). We find that in $\lesssim 3$ per cent of cases more than 10 per cent of the bound baryons lie outside our aperture. While bound material outside of the aperture could modify observable in some galaxies, this effect is insignificant in the majority of galaxies. Primarily, a consistent choice of aperture allows us to isolate aperture effects from other influences. Each galaxy is processed in isolation; therefore, there is no contribution from other structures along the line of sight. We use the same selection of EAGLE galaxies as T15, processing galaxies with stellar masses of $M_{\star} \geq 1.81 \times 10^{8} \mathrm{M}_{\odot}(\sim 100$ star particles at standard resolution).

\subsection{GAMA and SDSS survey data}

The GAmA survey (Driver et al. 2009; Robotham et al. 2010; Driver et al. 2011) is a spectroscopic and photometric survey of five independent sky fields, undertaken at the Anglo-Australian Telescope, and using the $2 \mathrm{dF} / \mathrm{AAOmega}$ spectrograph system. The three equatorial fields we consider follow up targets from the SDSS DR7 (York et al. 2000; Abazajian et al. 2009), yielding a sample of $\sim 190000$ galaxies with SDSS ugriz photometry (Hill et al. 2011; Taylor et al. 2011) with spectra covering the wavelength range 3700-8900 , with a resolution of $3.2 \AA$ (Sharp et al. 2006; Driver et al. 2011). The GAMA survey strategy provides high spectroscopic completeness (Robotham et al. 2010) and accurate redshift determination (Baldry et al. 2014) for these galaxies, above an extinction-corrected $r$-band Petrosian magnitude limit of 19.8. The galaxy stellar mass estimates and rest-frame photometry for the GAMA sample used in this paper are taken from Taylor et al. (2015).

Emission line indices in GAMA were measured assuming single Gaussian profiles, a common redshift for adjacent lines, and a stellar continuum correction simultaneously fit to each spectrum around the measured lines, as described by Hopkins et al. (2003) and Gunawardhana et al. (2013, 2015). Emission line fluxes are corrected for stellar absorption as described by Hopkins et al. (2003). Dust corrections are obtained using the stellar absorption-corrected Balmer emission line flux ratios, also described by Hopkins et al. (2003). The uncertainties associated with correcting Balmer lines for stellar absorption are discussed in both Hopkins et al. (2003) and Gunawardhana et al. (2013).

Derived $\mathrm{H} \alpha$ luminosities and SFRs are taken from Gunawardhana et al. (2013). Their emission line galaxies (ELGs) are initially selected to have $\mathrm{H} \alpha$ fluxes above the detection limit of $25 \times 10^{-20} \mathrm{~W} \mathrm{~m}^{-2}$ and a signal-to-noise ratio of $>3$, with AGNs identified and removed using standard $\left[\mathrm{N}_{\mathrm{II}}\right] \lambda 6584 / \mathrm{H} \alpha$ and [O III] $]$ 5007/H $\beta$ diagnostics (Baldwin, Phillips \& Terlevich 1981). The GAMA sample is supplemented with SDSS galaxies with detected $\mathrm{H} \alpha$ emission and signal-to-noise $>3$ from the MPA-JHU catalogue,${ }^{1}$ as the brightest ELGs observed by SDSS were not reobserved by GAMA.

Measurements of the $4000 \AA$ break (D4000) are also used in this work. For this, we compare to values measured directly from

\footnotetext{
${ }^{1}$ http://www.mpa.mpa-garching.mpg.de/SDSS/DR7/
}

the SDSS DR7 data (Strauss et al. 2002; Abazajian et al. 2009). We compare a stellar mass-matched sample of EAGLE galaxies to the publicly available SDSS D4000 values measured for the MPA-JHU ${ }^{1}$ catalogue using the code of Tremonti et al. (2004), with the index defined as in Bruzual (1983). For SDSS data, we use the mass estimates of Kauffmann et al. (2003a).

\section{DUST MODELLING WITH SKIRT}

Given a set of sources and a dust distribution, the SKIRT Monte Carlo code (Baes et al. 2003, 2011; Camps \& Baes 2015) follows the dust absorption and scattering of monochromatic 'photon packets' until they hit a user-specified detector, optionally calculating the heating and re-radiation of the dust grains including non-equilibrium stochastic heating. The position on the detector and wavelength of each arriving photon is registered, allowing a full integral field image of the galaxy to be constructed. Convolving this data cube with a filter yields mock fluxes.

The modular nature of SKIRT makes it straightforward to implement multiple source components and absorbing media using arbitrary spectral libraries and geometries. The choices and assumptions we make to represent the emissive and absorbing components of EAGLE galaxies in SKIRT are detailed in Sections 3.1 and 3.2. To represent the particle-discretized galaxies of EAGLE as continuous matter distributions for radiative transfer, particles are smoothed over some scale. For gas particles the SPH smoothing is used, while stellar smoothings are recalculated via a nearest neighbour search of star particles, as explained in Appendix A. While SKIRT is capable of very efficient processing, MCRT is fundamentally computationally expensive. We examine the issues of spectral resolution and convergence in Appendix B.

\subsection{SKIRT modelling: input SEDs}

The spectrum of a star particle in the simulation is assigned using SED libraries. Each particle is treated as an SSP with a truncated Gaussian emissivity profile, parametrized by a position, smoothing length and SED. SKIRT then builds a 3D emissivity profile through the interpolation of these individual kernels. The point of emission of individual photon packets is sampled from these kernels at user-specified wavelengths, and photon packets are launched assuming isotropic emission. For the optical wavelengths considered here, we neglect emission from dust and other non-stellar sources. Our representation of the source component for EAGLE galaxies, including subgrid absorption for the youngest stars, is detailed in Sections 3.1.1 and 3.1.2 below, and an example spectrum showing the different SED components is plotted in Fig. 1. Input SEDs and broad-band luminosities are stored for EAGLE galaxies, as described in Section 3.3.

\subsubsection{Old stellar populations}

Stellar populations with age greater than $100 \mathrm{Myr}$ are assigned GALAXEv (Bruzual \& Charlot 2003) SEDs as described by T15. Briefly, initial masses, (smoothed) metallicities and particle ages are extracted directly from the simulation snapshot. Absolute metallicity values, as opposed to those in solar units, are used to assign SEDs for the reasons given in section 3.1.2 of T15. Stars are assumed to form with a Chabrier (2003) IMF covering the stellar mass range of $[0.1,100] \mathrm{M}_{\odot}$, consistent with what is assumed in EAGLE. Star particle coordinates are also taken directly from the simulation output. Smoothing lengths specifying the width of the truncated Gaussian profile are determined using a nearest neighbour search, 


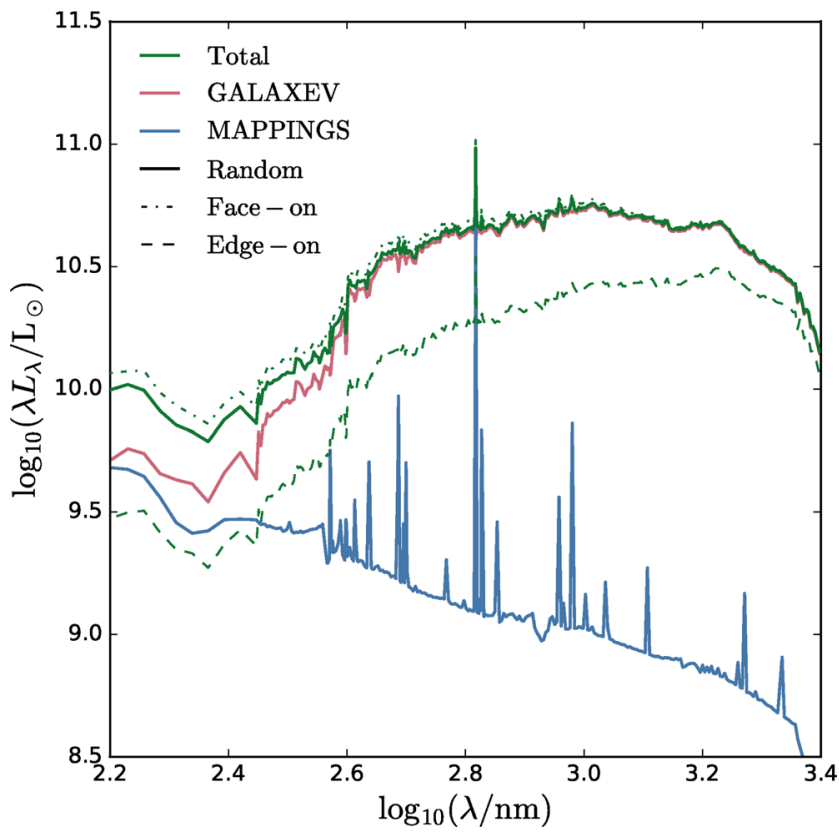

Figure 1. Example integrated rest-frame galaxy SED including ISM dust effects, generated for a late-type (S) EAGLE galaxy taken from simulation Ref100 (galaxy ID $=15814162$ in the EAGLE data base described by McAlpine et al. 2016). The green line indicates the total SED in the presence of dust. Solid lines show the integrated SED when the galaxy is observed along the $z$-direction of the simulation volume (an inclination angle of $50.2^{\circ}$ ), while dashed and dot-dashed lines denote edge and face-on views, respectively. The red and blue lines are the ISM dust-free contributions from the old stellar population $(t<10 \mathrm{Myr}$ ) modelled using GALAXEv (Bruzual \& Charlot 2003 ), and the young population ( $t>10 \mathrm{Myr}$ ) modelled using MAPPINGS-III (Groves et al. 2008), respectively. Properties and images of this galaxy can be found in Table 2 and Fig. 2, respectively. We see from the solid lines that the MAPPINGS-III SED contributes relatively more flux in the UV, and for some strong emission lines.

as detailed in Appendix A. Note that we do not explore alternative population synthesis models (e.g. Leitherer et al. 1999; Maraston 2005; Conroy, Gunn \& White 2009) in this paper; differences are expected to be small for the $z=0.1$ galaxies at optical wavelengths studied here (e.g. Gonzalez-Perez et al. 2014).

\subsubsection{Young stellar populations}

The treatment of the young stellar component is more involved due to two limitations of the simulation: (i) the relatively coarse sampling of star formation due to the limited mass resolution (see Table 1) and (ii) the inability of resolving birth cloud absorption associated with recent star formation. Though the diffuse ISM dust can be traced by enriched cold gas, the birth clouds of stellar clusters exhibit structure on sub-kpc scales (e.g. Jonsson et al. 2010), below the resolution limit of EAGLE. Such birth clouds are thought to disperse on time-scales of $\sim 10 \mathrm{Myr}$ (e.g. Charlot \& Fall 2000). To treat birth cloud absorption, we use the MAPPINGS-III spectral models of Groves et al. (2008), which track stars younger than $10 \mathrm{Myr}$, and include dust absorption within the photodissociation region (PDR) of the star-forming cloud, following the methodology of Jonsson et al. (2010). We therefore have two sources of dust: that associated with birth clouds which is modelled using MAPPINGS-III, and ISM dust whose effects we model using SKIRT.
We use an extended version of the re-sampling procedure of T15 to mitigate the effects of coarse sampling. Recent star formation is re-sampled in time over the past $100 \mathrm{Myr}$, from both star-forming gas particles and existing star particles younger than $100 \mathrm{Myr}$, as follows. The stellar particle stores the gas density of its parent particle, which is used to compute an SFR. We use this rate for young stars, and the SFR of star-forming gas particles, to compute how much stellar mass these particles formed on average over the past $100 \mathrm{Myr}$ (assuming a constant SFR). We then randomly sample this stellar mass in terms of individual star-forming regions, with masses that follow the empirical mass function of molecular clouds in the Milky Way (Heyer, Carpenter \& Snell 2001),

$\frac{\mathrm{d} N}{\mathrm{~d} M} \propto M^{-1.8}$ with $M \in\left[700,10^{6}\right] \mathrm{M}_{\odot}$.

For each particle re-sampled, sub-particle masses are drawn from this distribution until the mass of the parent particle is exceeded. Rejecting the last drawn sub-particle, the masses of sub-particles are rescaled such that the sum of their masses, $\Sigma m_{\mathrm{r}}$, exactly matches that of the parent. Sub-particles are then stochastically assigned formation times using the SFR of the star-forming particle. In this way, we replace star-forming gas particles, and young star particles, by a distribution of star-forming molecular clouds with the same total mass and spatial distribution as the original set of particles.

Stellar populations re-sampled with ages in the range $10 \mathrm{Myr}$ $\leq t_{\text {age }}<100$ Myr are assigned GALAXEV spectra, parametrized in the same way as in Section 3.1.1. They inherit the position and smoothing length of the particle from which they are sampled. These smoothing lengths are assigned to parent star and gas particles differently, as described in Appendix A.

Those re-sampled to have ages in the age range $t_{\text {age }} \leq 10 \mathrm{Myr}$ are assigned the MAPPINGS-III spectra of Groves et al. (2008). One caveat with using these spectral models is that the intrinsic spectra of stars used to model the spectra are specified by the Leitherer et al. (1999) (SB99) population synthesis models. This leads to some inconsistency in the modelling of the intrinsic stellar spectra, which come from GALAXEV for older populations. However, these differences are small in the optical ranges considered here (e.g. see Gonzalez-Perez et al. 2014). Another caveat is that a Kroupa (2001) IMF is assumed for these spectral models rather than that of Chabrier (2003), though again the differences in optical properties are minimal.

The MAPPINGS-III spectral libraries represent $\mathrm{H}_{\text {II }}$ regions, and their emerging spectrum therefore already treats the reprocessing of star light by dust in the star-forming region. In other words, birth cloud dust absorption and nebular emission are included in the SKIRT input spectra before any SKIRT radiative transfer is performed. We describe below how we avoid double counting dust in $\mathrm{H}$ II regions.

The MAPPINGS-III SEDs are parametrized as follows:

(i) SFR $\left(\dot{M}_{\star}\right)$ : The MAPPINGS-III spectra assume a given (constant) SFR between 0 and $10 \mathrm{Myr}$; the SFR assigned to a particle of initial mass $m_{\mathrm{r}}$ is given by $\dot{M}_{\star}=10^{-7} m_{\mathrm{r}} \mathrm{yr}^{-1}$, in order to conserve the mass in stars.

(ii) Metallicity $(Z)$ : The metallicities are specified by the SPHsmoothed absolute values of the simulation snapshot.

(iii) Pressure $\left(P_{0}\right)$ : The ambient ISM pressure, $P_{0}$, is calculated from the density of the gas particle from which the sub-particle is sampled. This is not directly accessible in EAGLE, but can be estimated using the polytropic equation of state that limits the pressure for star-forming gas (Dalla Vecchia \& Schaye 2012). Because the simulation snapshot contains the density at which each star particle 
formed, this estimator can be used for re-sampling both star-forming gas and young stellar particles.

(iv) Compactness $\left(\log _{10} C\right)$ : The compactness, $C$, is a measure of the density of an $\mathrm{H}_{\text {II }}$ region. This is calculated using the following equation from Groves et al. (2008):

$\log _{10} C=\frac{3}{5} \log _{10} \frac{M_{\mathrm{cl}}}{\mathrm{M}_{\odot}}+\frac{2}{5} \log _{10} \frac{P_{0} / k_{\mathrm{B}}}{\mathrm{cm}^{-3} \mathrm{~K}}$,

where $M_{\mathrm{cl}}$ is the star cluster mass taken to be the re-sampling mass $m_{\mathrm{r}}, P_{0}$ is the $\mathrm{H}$ II region pressure taken to be the particle pressure above and $k_{\mathrm{B}}$ is Boltzmann's constant. The parameter $C$ predominately affects the dust temperature and thus the FIR part of the SED, and therefore has little effect on the results presented here, see Camps et al. (2016) for a thorough discussion on how $C$ affects FIR colours of EAGLE galaxies.

(v) PDR covering fraction $\left(f_{\mathrm{PDR}}\right)$ : The PDRs associated with $\mathrm{H}$ II regions are influenced by processes well below the resolution of EAGLE. PDRs disperse over time as $\mathrm{O}$ and A stars die out. We assume a fiducial value of $f_{\mathrm{PDR}}=0.1$ for the PDR covering factor, which can be compared to the 'typical' value of $f_{\mathrm{PDR}}=0.2$ used by Groves et al. (2008) and Jonsson et al. (2010), following the calibration presented by Camps et al. (2016).

With the parameters of the STARBURST SEDs determined, the SKIRT source emissivity profile is then set. As explained by Jonsson et al. (2010), the scale of the $\mathrm{H}$ II region emissivity profile should be set so as to enclose a similar mass of ISM to that required to be consistent with the subgrid absorption. Doing so avoids double-counting the dust in the subgrid absorption (which already affects the source spectra) and dust absorption in the diffuse ISM modelled by SKIRT. To approximate this, we assume a fixed mass for the re-sampled particles of $M_{\mathrm{PDR}}=10 M_{\mathrm{cl}}$ (e.g. Jonsson et al. 2010), and set the corresponding size of the region to be $r_{\mathrm{HII}}=\sqrt[3]{8 M_{\mathrm{PDR}} / \pi \rho_{\mathrm{g}}}$ ( for a cubic spline kernel), with $\rho_{\mathrm{g}}$ the local gas density, taken from the parent particle. This is taken to be the smoothing length of MAPPINGS-III sources. As noted previously, the MAPPINGS-III model assumes the presence of birth cloud dust and that needs to be accounted for to ensure that the total dust mass is conserved. We budget for this additional dust using the ISM dust distribution, as described in Section 3.2.2. H II region positions are sampled within a kernel of size $r_{\mathrm{s}}=\sqrt{r_{\mathrm{p}}^{2}-r_{\mathrm{HII}}^{2}}$, with $r_{\mathrm{p}}^{2}$ being the parent kernel smoothing length, and about the parent particle position. This is such that in the infinite sample limit the net kernel of the $\mathrm{H}$ II regions is equivalent to that of the parent. Again, the smoothing lengths of gas and star parent particles are obtained differently, as explained in Appendix A. Finally, those sub-particles that are not converted to either a stellar or $\mathrm{H}$ II region source over the re-sampling period are reserved for the absorbing component to ensure mass conservation. Absorption in the (diffuse) ISM is modelled as described in Section 3.2 below.

\subsection{SKIRT modelling: observed properties}

Having detailed the parametrization of the source components, we proceed to describe the modelling of dust in the diffuse ISM. This dust component is mapped to an adaptively refined (AMR) grid, for which the optical depth of each cell is calculated at a given reference wavelength. Neglecting Doppler shifts, this enables the computation of the dust optical depth at any other wavelength once the wavelength dependence of the dust attenuation is specified. Details of the modelling of the dust and gas contents are given in Sections 3.2.2 and 3.2.1, respectively.

\subsubsection{Discretization of the ISM}

Dust in galaxies exhibits structure on a range of scales, from galaxywide dust lanes to sub-kpc 'dark clouds', with significant absorption across the range, down to the scale of molecular clouds (e.g. Hunt \& Hirashita 2009). We cannot resolve sub-kpc dust structures in EAGLE, which is why we include such small-scale dust via the source model of $\mathrm{H}$ II regions, as described in Section 3.1.2. We use the gas particles in EAGLE galaxies to estimate how dust is distributed in the diffuse ISM, and use SKIRT to calculate obscuration by this dust, as follows.

We discretize the gas density on the AMR grid using the octree algorithm (Saftly et al. 2013). A cubic root cell of size $60 \mathrm{pkpc}$ is created, centred on the galactic centre of mass, to capture all galactic material (see Section 2.2), and is refined based on the interpolated dust density derived from the gas particles, between a specified minimum and maximum refinement level. We increase the refinement level until the photometry is converged. Clearly, the minimum cell size should be smaller than the approximate spatial resolution of EAGLE to best capture ISM structure in the simulated galaxies. We find that a maximum refinement level of 9 (corresponding to a finest cell of extent $60 \mathrm{kpc} / 2^{9}=0.11 \mathrm{kpc}$ or $\approx 1 / 6$ of the $z=0$ gravitational softening), provides a grid structure that yields converged results when combined with a cell splitting criterion ${ }^{2}$ of $2 \times 10^{-6}$. We therefore adopt a maximum refinement level of 9 for our analysis, together with a minimum refinement level of 4 . While we use a minimum cell size twice as large as that of Camps et al. (2016), we have verified that this has a negligible effect on our results in the optical and NIR, while increasing the speed of our SKIRT simulations.

\subsubsection{Dust model}

Dust traces the cold metal-rich gas in observed galaxies (e.g. Bourne et al. 2013). Here we assume that the dust-to-metal mass ratio is a constant,

$f_{\text {dust }} \equiv \frac{\rho_{\text {dust }}}{Z \rho_{\mathrm{g}}}=0.3$,

where $Z$ is the (SPH-smoothed) metallicity, and $\rho_{\text {dust }}$ and $\rho_{\mathrm{g}}$ are the dust and gas density, respectively. The numerical value was determined by calibrating FIR properties of EAGLE galaxies by Camps et al. (2016), and is consistent within the uncertainties of observationally inferred values (e.g. Dwek 1998; Draine et al. 2007). The assumption of a constant value of $f_{\text {dust }}$ is common and is observed to apply to a wide variety of environments (e.g. Zafar \& Watson 2013; Mattsson et al. 2014), though there are indications it can vary in some cases (e.g. De Cia et al. 2013; Feldmann 2015). We implement this constant ratio by assigning a dust mass of $m_{\text {dust }}=f_{\text {dust }} m_{\mathrm{g}}$, where $m_{\mathrm{g}}$ is the particle mass. We use the dust model described by Zubko, Dwek \& Arendt (2004); a multicomponent dust mix tuned to reproduce the abundance, extinction and emission constraints on the Milky Way. Following Camps et al. (2016), gas must be either star forming (i.e. assigned a non-zero SFR by the simulation or in the re-sampling procedure) or sufficiently cold (with temperature $T<8000 \mathrm{~K}$ ) to contribute to the dust budget.

To account for the dust mass already associated with birth clouds when using the MAPPINGS-III source SEDs, we introduce 'ghost' particles that contribute negatively to the local dust density. These ghost particles are placed at the location of each $\mathrm{H}_{\text {II }}$ region, have a mass

\footnotetext{
${ }^{2}$ This is the maximum fraction of the total dust mass that can be contained within a single dust cell. If the cell contains a larger fraction and is below the maximum refinement level, the cell is subdivided.
} 
of $M_{\mathrm{PDR}}=10 m_{\mathrm{r}}$, where $m_{\mathrm{r}}$ is the stellar mass formed in the starforming region, and a smoothing length equal to three times that of the $\mathrm{H}_{\text {II }}$ region. The assumption that the PDR mass, $M_{\mathrm{PDR}}$, is 10 times that of the stellar mass formed follows the recommendation of Groves et al. (2008), the greater smoothing of the ghost contribution avoids negative dust densities. The creation of 'holes' in the dust distribution around $\mathrm{H}_{\text {II }}$ regions may seem unphysical, as observed $\mathrm{H}_{\mathrm{II}}$ regions are typically embedded in the densest (and dustiest) ISM. However, we have tested an alternative implementation where the dust mass of all contributing particles are downscaled to balance the additional dust invoked by the MAPPINGS-III SEDs, and find little perceptible difference in the results presented here. We will see that ISM dust obscuration is still higher around young stars, even in the presence of these ghost particles.

\subsection{Data products}

This section describes the data products that are generated by SKIRT. We reiterate that we do not consider kinematics when using SKIRT, i.e. no Doppler shifts are yet accounted for beyond any line broadening present in the input SEDs. We perform a convergence test in Appendix B to determine how to best sample the SED, both in terms of wavelength sampling and photon-packet sampling. We construct integrated spectra for all simulated galaxies in three orientations; edge on, face on and randomly orientated with respect to the galactic plane. The calculation of orientations for EAGLE galaxies is described in Section 4 below. The data products produced include the following:

(i) Integrated spectra capture all the photon packets emanating from the mock galaxy for the fixed list of specified wavelengths, and in a given direction. The standard resolution spectra consist of 333 wavelengths in the range $0.28-2.5 \mu \mathrm{m}$, chosen to sample the rest-frame ugrizYJHK photometric bands (see Appendix B for details). Spectra are produced with and without ISM dust at redshifts $z=0$ and redshift $z=0.1$ (the snapshot redshift from which the galaxies were selected). An example integrated rest-frame SED of a star-forming galaxy and including dust attenuation is plotted in Fig. 1.

(ii) Data cubes, or mock IFU data, consist of $256 \times 256$ spatial pixels, each with a spectrum at standard spectral resolution. Given that the field of view corresponds to $60 \mathrm{pkpc}$ on a side, this corresponds to $234 \mathrm{pc}^{\text {pixel }}{ }^{-1}$. Images are produced in both the rest and observed frames, but only for dust attenuated galaxies with $M^{\star}>10^{10} \mathrm{M}_{\odot}$. Again, these do not include kinematic effects, which will be the focus of future work.

(iii) Broad-band photometry The fluxes through the ugrizYJHK filters are obtained by convolving the integrated spectra with the filter transmission curves (transmission curves were taken from Doi et al. 2010; Hewett et al. 2006). We compute both rest-frame and observed-frame photometry for the entire galaxy sample both with and without ISM dust attenuation.

(iv) Broad-band images are produced by integrating along the wavelength axis of the data cubes. These are generated including dust for the ugriz SDSS bands, and provided in three colour portable network graphic format ${ }^{3}$ via the approach of Lupton et al. (2004). Fig. 2 shows three-colour gri images at $z=0.1$ for three different

\footnotetext{
${ }^{3}$ Note that these images are initially intended for illustrative purposes only, as the detailed light distributions are dependent on the somewhat ad hoc choice of stellar smoothing (similarly demonstrated by Torrey et al. 2015). While we find the influence of smoothing to be small for the results presented
}

galaxies and three orientations. We picked a late-type, an irregular and an early-type galaxy. Some properties of these galaxies are listed in Table 2. Structural features resembling spiral arms and tidal tails are distinguishable for the late and irregular types, respectively, while the early type exhibits a smooth, featureless light distribution. Star-forming $\mathrm{H}$ II regions appear pink due to $\mathrm{H} \alpha$ emission in the MAPPINGS-III SEDs for these $z=0.1$ galaxy observations. We also observe scattering and absorption by dust for the late and irregular types.

Data products will be made available via the EAGLE public data base (McAlpine et al. 2016), with the exception of data cubes, which are available through collaboration with the authors. ${ }^{4} \mathrm{We}$ will below compare the output from the SKIRT simulations to those with the same source model but without obscuration by ISM dust. Note, however, that the MAPPINGS-III source model always includes dust associated with the birth cloud. We will refer to these models as 'ISM dust-free' in what follows.

\section{ATTENUATION PROPERTIES OF SKIRT GALA XIES}

In this section, we focus on how attenuation depends on galaxy orientation at low redshift $(z=0.1)$. This helps us to separate the effects of geometry and dust content, and facilitates the interpretation of a comparison with observations.

\subsection{Broad-band attenuation}

The orientation of a galaxy can profoundly affect its measured colours, particularly in the case of thin spiral galaxies where the edge-on view is much more affected by dust than the face-on view. Indeed, the reddest galaxies observed in the local Universe are often edge-on spirals (e.g. Sodre, Ribeiro da Silva \& Santos 2013). The SKIRT modelling naturally accounts for this effect, as opposed to the two-component screen model presented in T15 which relies on simple geometrical arguments to account for this. To quantify orientation effects in disc galaxies, we use three lines of sight: parallel, perpendicular and randomly oriented with respect to the galactic plane. This helps constrain orientation effects on dust extinction for each galaxy individually, as well as providing a set of photometry with random orientations used when comparing to data.

We assume that the disc of a galaxy is perpendicular to the spin vector, $\boldsymbol{S}$, of its stars. We calculate $\boldsymbol{S}$ by summing the spin vectors of all star particles within a shell with inner and outer radii of 2.5 and $30 \mathrm{pkpc}$, respectively, in the centre-of-mass rest frame of the galaxy. The outer radius corresponds to the maximum radius of a galaxy assumed in Section 2.1; the inner radius was chosen to avoid a significant contribution from a bulge or regions strongly affected by gravitational softening. We found that with this selection, $S$ is generally dominated by the dynamically cold rotating disc component, if present. We characterize the orientation of a galaxy by its inclination angle $\theta$, such that face-on galaxies have $\cos (\theta)=1$.

In Fig. 3 we plot the attenuation in the $B$ band, $A_{B}$, as function of orientation, for all EAGLE galaxies from simulation Ref-100 with

in this paper (see Appendix A), analysing the influence smoothing has on morphologies is left to a future work.

${ }^{4}$ To access the data base and receive updates on its content, register at http://icc.dur.ac.uk/Eagle/database.php. 


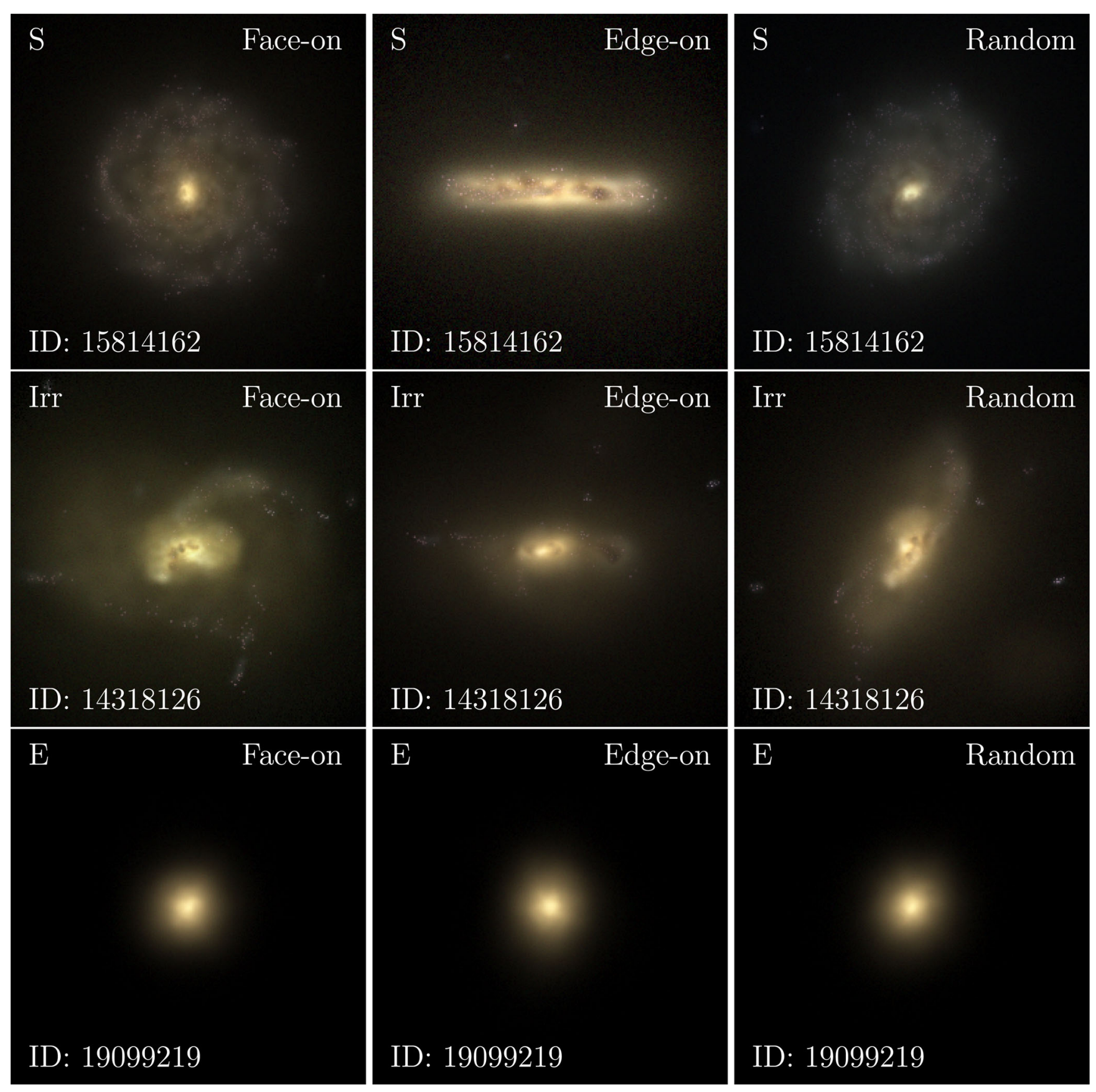

Figure 2. Observer frame true colour gri images of selected EAGLE galaxies from Ref-100 at $z=0.1$, including dust obscuration. The galaxy ID can be used to identify each galaxy on the EAGLE data base (McAlpine et al. 2016), see also Table 2. Rows from top to bottom show a late-type spiral (Hubble type S), an irregular type (Irr) and an early-type (E) galaxy, with columns from left to right showing face-on view (parallel to the stellar angular momentum axis), edge-on view and the view along the $z$-axis of the simulation volume ('random'). Images are produced using $4 \times 10^{6}$ photon packages at each of 333 wavelengths, spaced as described in Appendix B. H II regions appear pink in the colour scheme, due to strong $\mathrm{H} \alpha$ emission at $\sim 6700 \AA$ red-shifted into the $i$ band, dust lanes are clearly visible in the $\mathrm{S}$ and Irr images. The kinematic orientation works well for the spiral galaxy, but the disordered gas distribution caused by an ongoing merger event in the Irr case yields more absorption in the face-one view than in the edge-on view. Images are $60 \mathrm{pkpc}$ on a side.

stellar mass $M_{\star}>10^{10} \mathrm{M}_{\odot}$; points represent individual galaxies coloured by intrinsic $u^{\star}-r^{\star}$ ( ${ }^{\star}$ denotes intrinsic photometry). Two sequences are observed: (i) a broad sequence of intrinsically blue (star-forming) galaxies where $A_{B}$ increases with decreasing $\cos (\theta)$ and (ii) a very tight sequence of intrinsically red (passive) galaxies showing no orientation dependence. Such a dichotomy is of course unsurprising: red galaxies typically have low cold gas fractions and therefore negligible dust attenuation, whereas star-forming (intrinsically blue) galaxies have relatively high cold gas fractions, with gas distributed in a disc; hence, dust obscuration is higher and depends on orientation.

The median $A_{B}$ of intrinsically blue galaxies with $A_{B} \geq$ 0.05 in bins of $\cos (\theta)$ is plotted as black squares in Fig. 3), with the grey region enclosing the 16 th-84th percentiles. The median attenuation $A_{B}$ of EAGLE galaxies increases from 0.3 to $\geq 0.6$ from face-on to edge-on orientation but the scatter 


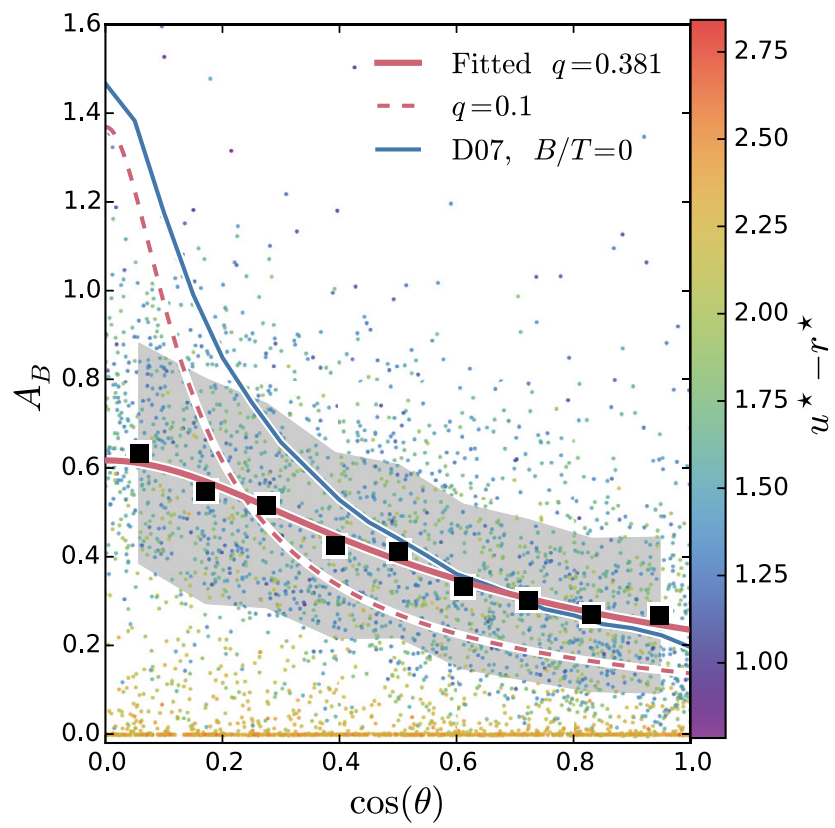

Figure 3. The Johnson rest-frame $B$-band attenuation, $A_{B}$, as a function of inclination angle; $\cos \theta=1$ corresponds to face on. Points are individual EAGLE galaxies from simulation Ref-100 at $z=0.1$, with $M_{\star}>10^{10} \mathrm{M}_{\odot}$, coloured by ISM dust-free $u^{\star}-r^{\star}$ colour. Black squares indicate the median relation for galaxies with $A_{B} \geq 0.05$, binned by $\cos (\theta)$. The grey, shaded region indicates the 16 th-84th percentile range in each bin. The red, solid line shows the best fit of the form equation (4), which has $q=0.396$. The red, dashed line shows the same fit, except with $q=0.1$ to represent a thinner discs, which follows the trend of the intrinsically blue galaxies better. Galaxies with $A_{B}<0.05$ are typically red in $u^{\star}-r^{\star}$ and occupy a tight distribution in $A_{B} \approx 0$, independent of orientation. For comparison, we also overlay the observed relation from Driver et al. (2007) (D07 in the legend) for the median attenuation curve of the disc component only, of galaxies with bulge/total ratio smaller than 0.8 .

around the trend is large $(\approx 0.5 \mathrm{mag})$. We fit the angular dependence of the median relation using the ellipsoidal dust model discussed by $\mathrm{T} 15$,

$A_{B}(\cos \theta)=a \frac{q}{\sqrt{q^{2}+\left(1-q^{2}\right) \cos ^{2} \theta}}$,

where $a$ is the edge-on obscuration and $q$ represents the axial ratio of the galaxy; lower $q$ corresponds to thinner galaxies. We treat $a$ and $q$ as free parameters in the fit, and plot the fitted curve in red. The functional form fits the trend well for an axial ratio of $q=0.381$. The scatter around the median likely originates from both diversity in the ISM distribution in different galaxies, i.e. deviation from an idealized disc, but also from errors in identifying the correct orientation of the disc plane. Indeed, we showed in Fig. 2 that the galactic plane is not always easily defined, as evidenced by the irregular galaxy shown in the middle row.

Driver et al. (2007) use a sample of galaxies from the Millennium Galaxy Catalogue with estimated bulge-to-total $(B / T)$ light ratios of $B / T<0.8$ to measure the extent to which the location of the 'knee' in the $B$-band luminosity function (Schechter 1976) depends on inclination. They fit their results with the model of Tuffs et al. (2004) to obtain the typical attenuation separately for bulge and disc components. We plot the relation of Driver et al. (2007) for a typical disc $(B / T=0)$ in Fig. 3 as a solid blue line.

The median EAGLE $A_{B}$ values (black squares) and the fitted form of equation (4) (red line) are consistent with those obtained by
Driver et al. (2007) for nearly face-on discs $(\cos (\theta) \geq 0.5)$, but are significantly lower for highly inclined discs. While there is uncertainty in the absolute values measured for $A_{B}$, as discussed by Driver et al. (2007), the difference between typical face- and edgeon $A_{B}$ values is better constrained ${ }^{5}$ and clearly significantly larger in the data compared to EAGLE. Note that the Driver et al. (2007) data are represented by a pure disc (blue line) for simplicity. While EAGLE spirals clearly possess bulges (see e.g. Fig. 2), the difference between face-on and edge-on attenuation found by Driver et al. (2007) varies little with $B / T$. The blue line provides a guide curve to highlight the smaller range in $A_{B}$ with inclination for EAGLE. Decomposition of SKIRT light profiles into bulge and disc contributions is left to a future study.

The $q=0.1$ curve, which models thinner discs for the same dust content as the fitted curve, demonstrates much better agreement with Driver et al. (2007) than the median EAGLE relation. This suggests that the discrepancy is likely due to EAGLE galaxies not being as thin as observed galaxies. The fact that EAGLE galaxies are thicker than observed is not only due to numerical resolution. Indeed, we compare the $A_{B}(\cos \theta)$ relation for galaxies taken from the three $(25 \mathrm{Mpc})^{3}$ simulations listed in Table 1 . These simulate the same volume, but at different resolutions. The number of galaxies with $M_{\star}>10^{10} \mathrm{M}_{\odot}$ in this smaller volume is $\lesssim 100$; therefore, the two sequences in the $A_{B}(\cos \theta)$ relation are not well constrained if we simply use the mock photometry of randomly oriented galaxies as we did in Fig. 3. We therefore calculate $A_{B}(\cos \theta)$ for all sufficiently massive galaxies $\left(M_{\star}>10^{10} \mathrm{M}_{\odot}\right)$ at 40 inclinations for each galaxy, equally spaced in $\cos (\theta)$, and plot the resulting curves in Fig. 4. Equation (4) is fit to the median relation and plotted as a dashed coloured line. While higher values of $A_{B}$ are seen in the higher resolution RefHi-25 and Recal-25 samples, the difference with respect to the median values of Ref- 25 is small. Neither the plotted curves for individual galaxies nor the fits using equation (4) to the median trend show strong evidence for $A_{B}$ being more sharply peaked at improved numerical resolution.

The weaker inclination dependence and lower edge-on values of $A_{B}$ in EAGLE are instead likely a consequence of EAGLE's subgrid physics, in particular the use of an imposed Jeans-limiting, polytropic relation for star-forming gas (Section 2.1). This relation yields a Jeans length at the star formation threshold of the $\sim 1.5 \mathrm{kpc}$, and EAGLE discs are unable to be much thinner than this. This relation is imposed to avoid numerical fragmentation below the resolution of the simulation, as explained by S15. Dust discs in observed galaxies, on the other hand, are much thinner, $\sim 100-200$ pc (e.g. Xilouris et al. 1999; De Geyter et al. 2014; Hughes et al. 2015). In a thin disc seen edge-on, the dust optical depth to young stars will be much higher than if the disc where thick, and this seems to be the main difference between observed and simulated galaxies.

This comparison demonstrates that the $A_{B}(\cos \theta)$ dependence displays both strong and weak convergence behaviour, with increased numerical resolution not changing the relation significantly - and not improving the agreement with the data. We show in Appendix A that reducing all star particles to point sources only boosts the edge-on value of $A_{B}$ by $\lesssim 0.1 \mathrm{mag}$. We conclude from this that the lower values for $A_{B}$ for edge-on EAGLE galaxies are likely a result of the simulations being unable to represent cold gas; the high column densities and clumpy structure of molecular gas observed in

\footnotetext{
${ }^{5}$ Driver et al. (2007) derive the relative attenuation directly by measuring how the knee position of the luminosity function differs for edge-on and face-on galaxies.
} 

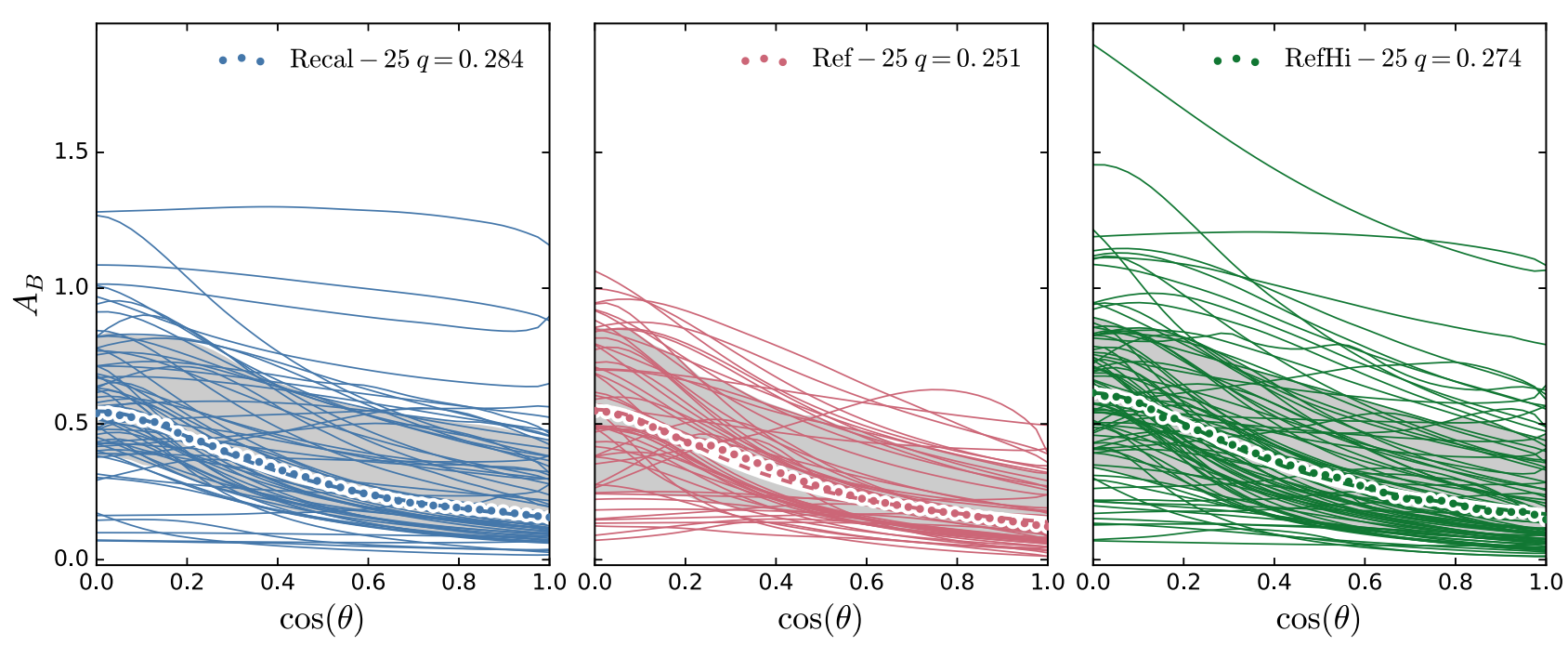

Figure 4. Johnson rest-frame $B$-band attenuation as function of orientation for EAGLE galaxies, taken from simulations Recal-25, Ref-25 and RefHi-25 at $z=0.1$ (left-hand to right-hand panels, see Table 1 for details). Thin curves show $A_{B}$ for individual galaxies as a function of inclination, the shaded circle represent the median trend and the grey region includes the 16th-84th percentiles. The dashed line approximately overlying the circles represents the fit of equation (4); the best-fitting value of $q$ is indicated in each panel. We see significant galaxy-to-galaxy variation in the shape of the attenuation-inclination relation, with the highest attenuation values in the higher resolution galaxies, but little difference between the trend of the median inclination as a function of $\cos (\theta)$.

real disc galaxies is not reproducible in the EAGLE simulations without realistic modelling of gas with $T \lesssim 10^{4} \mathrm{~K}$. The influence that thicker discs (and thus lower edge-on attenuation) has on our results is discussed further below.

\subsection{Broad-band colour effects}

The extent to which inclination affects the optical colour distribution of EAGLE galaxies with $10^{10} \mathrm{M}_{\odot} \leq M_{\star}<10^{11} \mathrm{M}_{\odot}$ is illustrated in Fig. 5, where we plot intrinsic (ISM dust-free) $g^{\star}-r^{\star}$ colour against $g-r$ in the presence of dust. In the left-hand panel, we shade regularly spaced colour-colour bins by the median value of $\cos \theta$ of galaxies in that bin (provided the bin contains more than 10 galaxies). We see a clear trend in attenuation with inclination, especially for intrinsically blue galaxies of $g^{\star}-r^{\star} \lesssim 0.6$, with galaxies possessing median $\cos (\theta)$ values of $\approx 0.25$ and $\approx 0.65$ for maximal and minimal offsets from the $1: 1$ relation, respectively. For galaxies with redder intrinsic colours, the trend is less pronounced and the maximal offset is lower, as expected for less dusty galaxies.

In the right-hand panel of Fig. 5, we plot logarithmically spaced contours representing the number of galaxies per colour-colour bin. Intrinsically red $\left(g^{\star}-r^{\star} \approx 0.75\right)$ galaxies follow the $1: 1$ relation closely with little offset, whereas intrinsically blue $\left(g^{\star}-r^{\star} \approx\right.$ $0.4)$ galaxies are offset to redder colours and show a large scatter. Worth noting is the approximately constant median offset to the red of $\approx 0.1$ mag for galaxies with $g^{\star}-r^{\star} \lesssim 0.6$, implying that a similar average reddening is experienced by star-forming galaxies regardless of SFR.

Some galaxies lie marginally below the $1: 1$ relation. In most cases, this is due to uncertainty in the photometry (see Appendix B), particularly in dust-free galaxies where the attenuation is small anyway, and these negative reddening measurements are $\sim 0.001$ mag. However in rare instances, measured for higher redshift EAGLE galaxies, significant negative reddening is observed. This can be attributed to those galaxies demonstrating heavy obscuration in their central regions, leading to higher contribution of young stars in the outskirts, conspiring to produce bluer colours overall

\subsection{Attenuation curves}

The extinction curve is an intrinsic property of a given dust grain population; combining the wavelength-dependent cross-sections of absorption and scattering. Our choice of dust mix thus sets the optical depth of dust cells modelled by SKIRT. However, the extinction does not provide a direct mapping between the intrinsic and observed SEDs, which additionally depends on the relative distribution of stars and dust and the orientation of the galaxy along the line of sight. This galaxy and line-of-sight specific mapping is referred to as the attenuation curve. One example of why the curves may differ significantly is that the young stars that dominate emission at short wavelengths are in general embedded in dusty regions and hence their blue light is more strongly dust attenuated.

As a result, the attenuation curves may differ systematically in shape from the extinction curve of the individual dust cells. The shape of the curve is also likely orientation dependent, for example stars in a central bulge may be obscured in edge-on but not face-on projections. As a result, the normalization, shape and orientation dependence of the attenuation curve are to some extent degenerate in observed integrated spectra when an attenuation proxy such as the Balmer decrement is used.

It is typical to assume a fixed shape of the attenuation curve to de-redden observed SEDs. Using the SEDs, we generate for EAGLE galaxies, we can explore the typical attenuation curves that arise from our MCRT treatment, and how these may vary systematically with orientation. While EAGLE galaxies appear to have thicker discs than observed (see Section 4.1), we hope to provide an indication of the ways in which real galaxy attenuation curves can vary from basic screen models using the comparatively realistic and diverse morphologies that arise in EAGLE galaxies. Studies of variation in galaxy attenuation curves have been performed for observed galaxies assuming idealized geometries by Byun et al. (1994), Baes \& Dejonghe (2001), Wild et al. (2011) and Kriek \& Conroy (2013), and for small samples of zoomed galaxy simulations by Natale et al. (2015). 


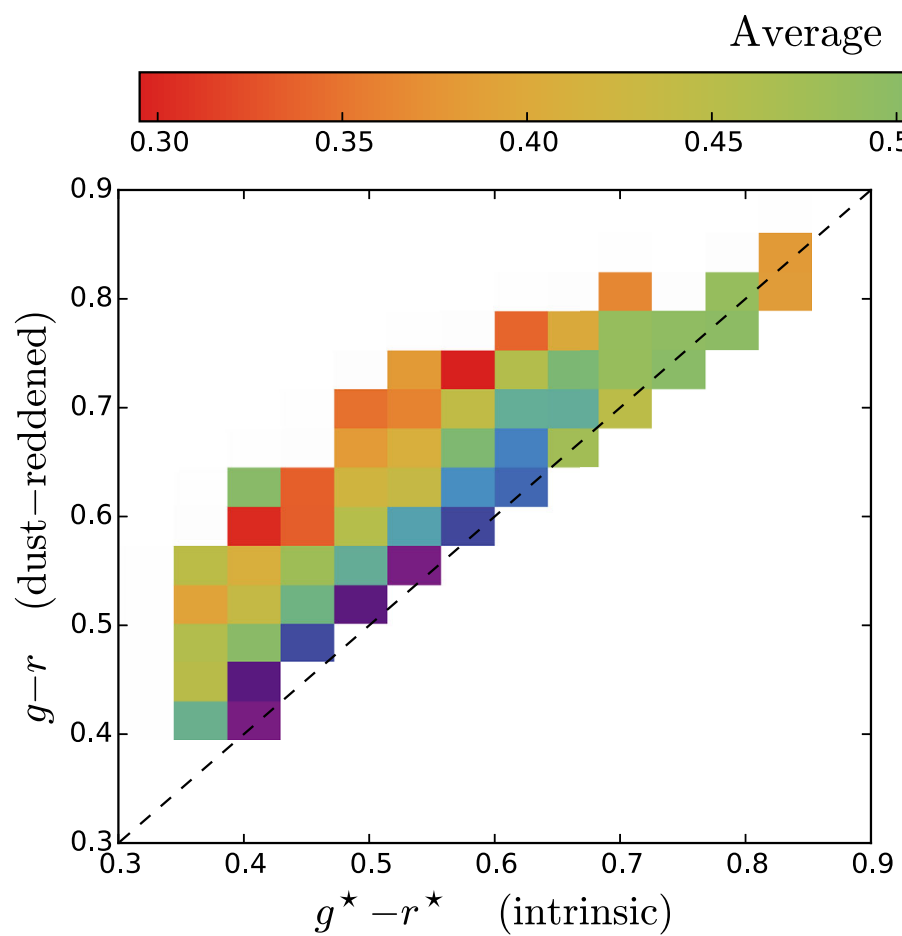

$$
\begin{aligned}
& 0.50 \\
& 0.8 \\
& 0.7 \\
& 0.9 \\
& 0.3 \\
& 0.3
\end{aligned}
$$

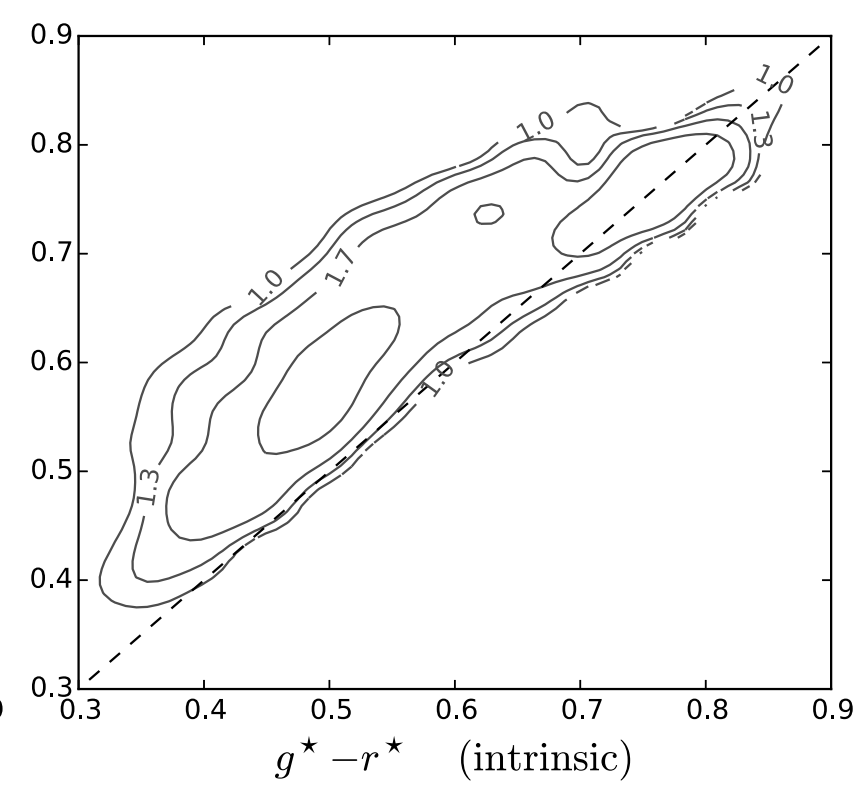

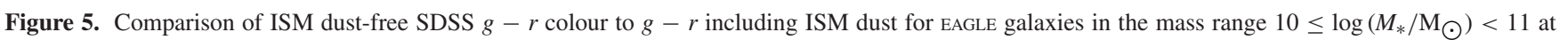
$z=0.1$; the black dashed line indicates the $1: 1$ relation in both panels. In the left-hand panel, the median value $\cos (\theta)$ is plotted in regularly spaced bins in colour (only bins with more than 10 galaxies are shown). The right-hand panel shows the number density $n$ of galaxies per colour-colour bin as grey contours labelled by $\log (n)$. There is a clear trend of increased reddening at higher $\cos (\theta)$ as expected. Some galaxies lie marginally below the $1: 1$ line, the reasons for which are discussed in the text.

We plot the $r$-band luminosity-weighted average attenuation curves of intrinsically blue $\left(u^{\star}-r^{\star}<2.0\right)$ EAGLE galaxies, normalized by the attenuation in the $V$ band, in Fig. 6. Face-on, edge-on and random projections are plotted as blue, red and green curves, respectively. Recall that dust in birth clouds is accounted for in our models by the MAPPINGS-III SEDs of Groves et al. (2008) for which we do not have the analytical description of the intrinsic attenuation curve. Therefore, we approximate the attenuation given the modelled dust content assuming a foreground screen. Fortunately, the proportion of optical light attenuated in the $\mathrm{H}_{\text {II }}$ or associated PDR regions is small relative to the diffuse component, except at some specific atomic transitions. Nevertheless, we find that the increased attenuation visible in Fig. 6 at the $\mathrm{H} \alpha$ and $\mathrm{H} \beta$ wavelengths is still clearly present even when only the diffuse contribution is taken into account: this is because PDR regions are preferentially embedded in denser regions of the ISM, and it is this diffuse ISM dust that causes the high attenuation. We emphasize that preferential attenuation of young stars due to dust in a birth cloud screen is explicitly built in to both the SKIRT and the Charlot \& Fall (2000) model employed by T15. The difference is due to additional preferential attenuation of the diffuse ISM represented as a single screen in Charlot \& Fall (2000).

In all cases, attenuation increases rapidly towards shorter wavelengths with significantly higher attenuation at certain discrete wavelengths and a broad absorption feature at $\approx 220 \mathrm{~nm}$. For face-on galaxies, the slope is much steeper than the intrinsic dust extinction law. The discrete wavelengths correspond to atomic transitions at which star-forming regions dominate emission, their boosted attenuation is due predominately to the increased diffuse dust around these regions. ${ }^{6}$ The feature at $\approx 220 \mathrm{~nm}$ is intrinsic to the assumed dust extinction law.

The average edge-on attenuation curve is less steep, or 'greyer', than that of face-on galaxies. This is because the dust in EAGLE galaxies is spatially correlated with star-forming gas; therefore, intrinsically blue stars are preferentially obscured in the face-on view, while in the edge-on view that dust also acts as a screen for older stars. The $A_{\lambda}$ curve for the randomly oriented values exhibits an intermediate steepness between the face-on and edge-on curves.

The extinction curve assumed by SKIRT when processing EAGLE galaxies is that of Zubko et al. (2004). The extinction curve is plotted as a black dotted line in the top panel (again normalized to 1 for the $V$ band), while in the bottom panel we plot the residuals of the EAGLE attenuation curves once the $V$-band normalized extinction is subtracted, to isolate the influence of geometry and orientation. We see that the EAGLE curves are steeper than the intrinsic extinction curve, again a manifestation of the preferential obscuration of young stars, most exaggerated for the face-on projection. We also see that the EAGLE curves lie above the extinction curve at NIR wavelengths. This can be ascribed to absorption overtaking scattering as the primary photon-dust interaction at wavelengths longer than optical, leading to a smaller fraction of attenuated light being scattered into the line of sight than at optical wavelengths. As a result, when curves

\footnotetext{
${ }^{6}$ Constructing ISM attenuation curves for the $\mathrm{H}$ II regions alone yields curves similar to the Calzetti et al. (2000) and Charlot \& Fall (2000), with the $\mathrm{H} \alpha$ feature reduced by $\approx 90$ per cent.
} 


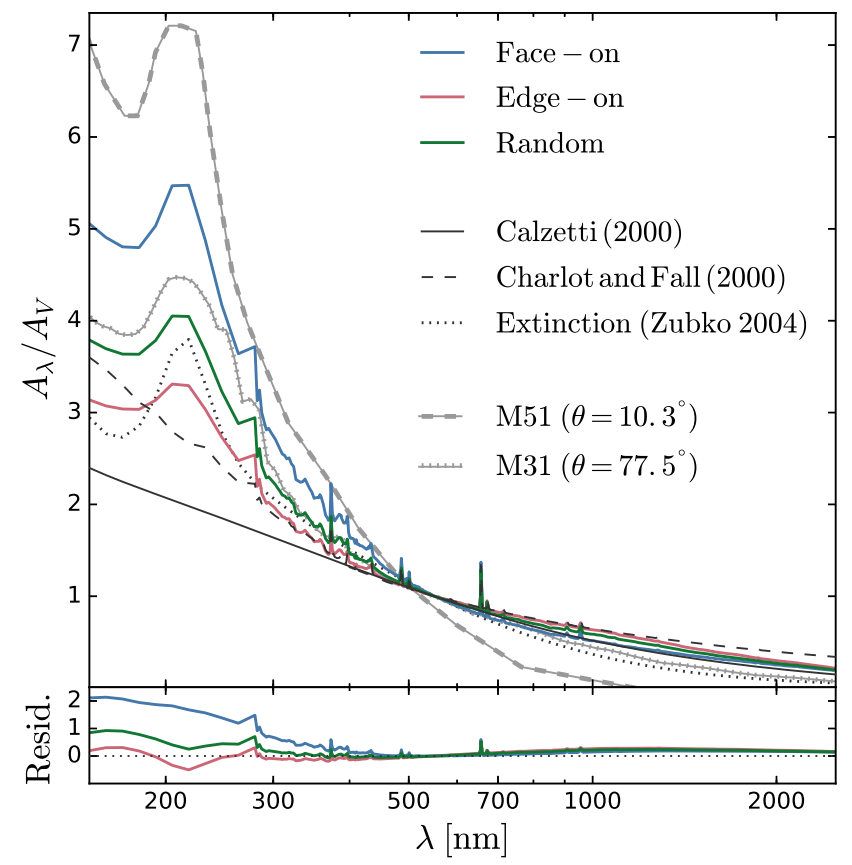

Figure 6. Attenuation as a function of wavelength, normalized to attenuation in the $V$ band at $550 \mathrm{~nm}$. The luminosity-weighted average attenuation curves of intrinsically blue $\left(u^{\star}-r^{\star}<2.0\right)$ EAGLE galaxies for face-on, edge-on and random orientations are plotted as blue, red and green curves, respectively. The attenuation laws of Calzetti et al. (2000) and Charlot \& Fall (2000) are plotted as solid and dashed black lines, respectively, and the attenuation curve inferred for the galaxy M51 by De Looze et al. (2014) and for M31 by Viaene et al. (2017), are plotted as dashed and dotted grey lines, respectively. The inclination of these galaxies is plotted in the legend. We also plot the intrinsic extinction curve used by SKIRT, Zubko et al. (2004). The bottom panel shows the residuals between the three EAGLE curves and the dust extinction curve of Zubko et al. (2004), assumed by SKIRT. The shape of the attenuation curve for EAGLE galaxies varies with orientation, with face-on galaxies exhibiting a stronger wavelength dependence.

are normalized at optical wavelengths, the NIR attenuation appears boosted relative to the pure extinction curve.

For comparison, we plot the attenuation curves of the Calzetti et al. (2000) and Charlot \& Fall (2000) screen models. Comparing to the screen-model curves, we see that the attenuation curves for EAGLE are generally steeper at all orientations. The screen models are closest to the edge-on curve at short wavelengths, due to dust behaving as a screen for many stars in an edge-on view. The Charlot \& Fall (2000) curve represents a two-component screen model, accounting for additional attenuation of young stars associated with stellar birth clouds. This age-dependent attenuation model provides better agreement with the EAGLE curves than the single screen Calzetti et al. (2000) model, laying closest to the edge-on EAGLE curve. The fact that young stars are also preferentially attenuated by diffuse ISM in our SKIRT modelling may explain why the EAGLE attenuation curves are steeper still.

We also plot attenuation curves derived for local galaxies M31 and M51 [from Viaene et al. (2017) and De Looze et al. (2014), respectively]; M31 is a relatively edge-on galaxy, with an inclination angle of $\theta=77^{\circ}$ (Brinks \& Burton 1984), whereas M51 is practically face-on at $\theta=10^{\circ}$ (De Looze et al. 2014). The M31 attenuation curve lies between the face-on and edge-on curves at wavelengths short-ward of the $V$ band, residing closest to the random projection curve. The M51 curve is steeper than any of EAGLE or screen-model curves. The M31 and M51 curves are both steeper than the EAGLE curves for comparable galaxy orientations. They also show more difference in slope than between the face-on and edge-on curves. We suggest that this is because EAGLE galaxy discs are thicker, and smoother than observed discs, both a consequence of limitations in the subgrid physics. This could indicate that the orientation dependence we identify in EAGLE galaxies may become stronger if EAGLE galaxies possessed more realistic, thinner discs.

Observational studies have explored attenuation curve variation through SED fitting of low- and high-redshift galaxy samples and assuming screen-like attenuation (e.g. Wild et al. 2011; Kriek \& Conroy 2013, respectively). Wild et al. (2011) find a similar trend between attenuation curve slope and inclination for nearby galaxies as we observe here. However, both Wild et al. (2011) and Kriek \& Conroy (2013) find a slight weakening of the $2175 \AA$ bump feature for face-on galaxies, which is not apparent in EAGLE. As this feature and its variation is attributed to poorly understood dust grain species that inhabit certain regions of galaxies, and given our modelling does not include spatial variation of the dust mix, this is perhaps unsurprising. A better understanding of the nature of these enigmatic dust grains, and their location in galaxies, would allow us to incorporate this into our modelling.

\section{SKIRT COLOURS OF EAgLE GALAXIES}

In this section we compare colours of EAGLE galaxies to GAMA data, as well as to the fiducial model of T15 (their GD+O model, which we will refer to as the 'T15' model below). We recall that the models we discuss have two sources of dust, that associated with birth clouds which is modelled by MAPPINGS-III, and ISM dust taking into account using SKIRT radiative transfer. We will sometimes refer to models without ISM dust as 'intrinsic' colours and to these galaxies as 'dust-free' but note that this only refers to ISM dust, not the dust associated with the MAPPINGS-III source model.

\subsection{Comparison with observations}

\subsubsection{Colour distribution at a given stellar mass}

The distribution of rest-frame galaxy $g-r$ colours at $z=0.1$, in narrow ( $0.3 \mathrm{dex})$ non-contiguous stellar mass bins, showing both $z=0.1$ EAGLE galaxies and GAMA data, is plotted in Fig. 7 (which can be compared to the simpler dust-screen model of T15, thin solid lines). Stepped lines represent the EAGLE colour histograms, using black to denote simulation Ref-100 and red to denote simulation Recal-25. These are either thin dashed to indicate colours without ISM dust, thin solid to represent the GD+O model of T15 or thick solid representing those obtained using radiative transfer with SKIRT. Continuous blue lines correspond to rest-frame (volume-limited) GAMA galaxy colours without dust correction from Taylor et al. (2015). Stellar masses for the GAMA galaxies, inferred through SED fitting, are taken from Taylor et al. (2011). All distributions are normalized to unit area. Here we compare the SKIRT to observed distributions, additionally comparing to the T15 model in Sections 5.1.2-5.2.2.

Comparing dashed to solid lines in the top panel demonstrates that dust reddening in massive $\left(10^{11.2}<M_{\star} / \mathrm{M}_{\odot}<10^{11.5}\right)$ blue galaxies is significant, with blue galaxies redder in SKIRT compared to intrinsic colours by $0.1-0.2$ mag in $g-r$, changing the bi-modal ISM dust-free colour distribution to a single red peak at $g-r$ $\approx 0.75$. The intrinsically blue colours of massive EAGLE galaxies is caused by relatively low levels of residual star formation, not completely suppressed by the AGN feedback. The dust content of 


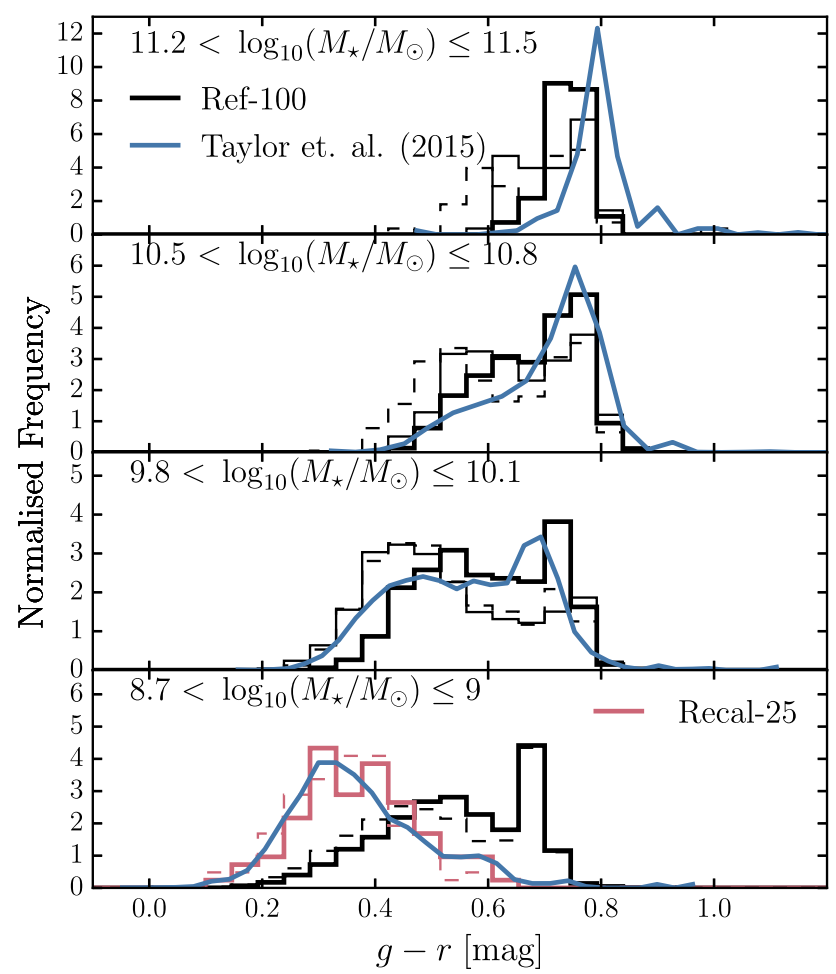

Figure 7. Rest-frame $z=0.1$ SDSS $g-r$ colour distributions of galaxies in four non-contiguous bins of stellar mass, labelled in each panel. EAGLE galaxies of simulation Ref-100 are shown in black, where dashed lines neglect ISM dust, thin solid lines indicate the T15 colours, thick solid lines include ISM dust modelled using SKIRT. Red lines (bottom panel only) are galaxies from the higher resolution simulation Recal-25, processed with SKIRT. Blue lines show the observed distributions of Taylor et al. (2015).

these star-forming regions is high, however, leading to significant dust reddening when processed with SKIRT. At these masses, about half of the galaxies on the SKIRT red sequence are dust reddened from an intrinsically bluer colour. ${ }^{7}$

Dust reddening also affects the $g-r$ colour of galaxies with masses $10^{10.5}<M_{\star} / \mathrm{M}_{\odot}<10^{10.8}$ strongly (second panel from top), shifting blue galaxies to higher $g-r$ by $\approx 0.1 \mathrm{mag}$, to $g-r \approx 0.6$, and changing the bi-modal colour distribution into a single red peak with a tail to bluer colours. This blue tail, due to galaxies with more moderate reddening, hints that the intrinsic colour distribution is in fact bi-modal. At these masses, about a third of the 'green valley' population with $g-r \approx 0.65$ comprises dust-reddened galaxies. The remaining galaxies have intrinsic colours that puts them in the green valley, and are typically transitioning between the blue and red populations, as discussed in detail by Trayford et al. (2016).

The second-lowest mass bin $\left(10^{9.8}<M_{\star} / \mathrm{M}_{\odot}<10^{10.1}\right)$ again contains a population of strongly reddened galaxies. A distinct

\footnotetext{
${ }^{7}$ Note that while the dust attenuation in EAGLE appears to be systematically lower than observed for edge-on galaxies (see Section 4.1), increasing attenuation would not necessarily lead to the EAGLE red sequence position shifting to even redder colours. Unlike in a screen model where extreme reddening is possible, in our SKIRT modelling galaxies with high dust content have colours that saturate to that of old stellar populations, as these populations are preferentially unobscured by dust. If the dust clouds are made optically thick, the galaxy photometry is essentially that of the unobscured population. More realistic attenuation values might, however, lead to more galaxies appearing as members of the red sequence.
}

bi-modality remains after reddening, with the red peak stronger than the blue peak, opposite to the case of intrinsic colours. Intrinsically blue galaxies appear less attenuated on average, with the blue peak shifted by only $\approx 0.05$ mag relative to the ISM dust-free photometry, to $g-r \approx 0.5$. The 'green valley' population is also boosted relative to the ISM dust-free photometry. Recalling Fig. 5, we see that the dust-boosted red and green galaxy populations produced by SKIRT have a tail to significantly bluer colours. The tail consists of galaxies that have little or no ISM dust as well as dusty galaxies seen nearly face-on with ISM dust-free colours typical of the star-forming population.

At the lowest stellar masses, $10^{8.7}<M_{\star} / \mathrm{M}_{\odot}<10^{9}$ (bottom panel), EAGLE galaxies show very little reddening when processed with SKIRT. Indeed, comparing the ISM dust-free and SKIRT $g-r$ distributions separately for the Ref-100 and Recal-25 simulations shows that dust effects are minimal.

In the most massive bin, observed colours from GAMA conform to a tight red sequence centred at $g-r \approx 0.7$. The SKIRT distribution is similar but shifted by $\approx 0.05$ mag to the blue. The median stellar metallicity of EAGLE galaxies agrees well with the observationally inferred values (S15), and the stars in these galaxies are generally old. It is therefore somewhat surprising that the simulated and observed colours do not agree better, since reddening is not important for these galaxies anyway (either in our model, or in the GAMA data). Trayford et al. (2016) showed that the metallicity distribution of star particles in EAGLE galaxies is nearly exponential, and it is the lower $Z$ particles that make EAGLE galaxies bluer than observed. A possible reason for the discrepant colours is thus that massive EAGLE galaxies have too low metallicities, even though the mass-weighted simulation metallicity agrees well with the luminosity-weighted observed metallicity, see Trayford et al. (2016) for more discussion.

The second most massive bin shows striking consistency between SKIRT and observed colours. The agreement with the data is in fact superior to that obtained with the dust-screen model of T15. In particular, the relative fraction of red and blue galaxies is much closer to the observed ratio when using the SKIRT. The reason for this is explored further below.

The second lowest mass bin shows similarly good agreement with the observed distribution, with SKIRT colours systematically shifted to somewhat redder values ( $\lesssim 0.05 \mathrm{mag}$ ). Again, the colours conform better to observation than those presented by $\mathrm{T} 15$, with the latter's dust-reddened colours in fact close to the intrinsic EAGLE colours.

Finally, in the lowest mass bin, the Ref-100 colours show poor agreement with observation. Furlong et al. (2015) showed that at these lower galaxy masses, numerical effects and poor sampling in EAGLE cause the SFRs to be too low and too many galaxies to be quiescent. We therefore also show the colours for the higher resolution Recal-25 simulation (red). These agree well with GAMA. The SKIRT colours of each of the simulations are very similar to those of T15, which is understandable as both are very close to the intrinsic colours (i.e. are subject to minimal reddening) in this mass range.

\subsubsection{Colour-mass diagram}

The $g-r$ colour versus stellar mass distribution of EAGLE galaxies is compared to that obtained from GAMA in Fig. 8. As explained above, the SFR and hence colour of low-mass galaxies in Ref-100 is not well resolved numerically (Furlong et al. 2015). We therefore combine the more massive EAGLE galaxies from Ref-100 with the 


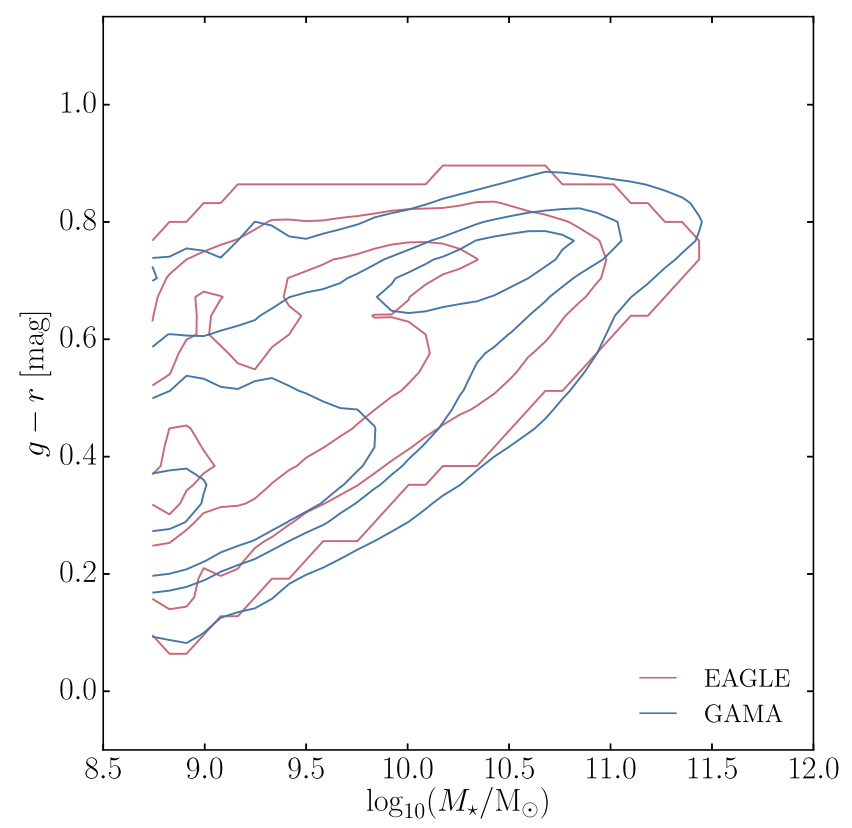

Figure 8. Rest-frame $z=0.1$ SDSS $g-r$ colour as a function of galaxy stellar mass, for qualitative comparison of EAGLE and GAMA. Red contours are for randomly oriented EAGLE galaxies, processed using SKIRT. The EAGLE sample combines galaxies taken from simulation Ref-100 at high mass with galaxies from simulation Recal-25 at lower stellar mass, to mitigate the poorer resolution of Ref-100 (see text). Blue contours show the observed distribution taken from Taylor et al. (2015). The contour levels from low to high exclude 3,18, 48 and 93 per cent of the galaxies in each sample.

low-mass galaxies from the higher resolution Recal-25 simulation, crossfading between the two in the mass range $10^{10}-10^{9} \mathrm{M}_{\odot}$ as in T15. The observed GAMA contours are based on the analysis of Taylor et al. (2015). Note that the crossfading between two simulations (performed as described in T15) extends the mass range over which galaxies are well resolved, but also introduces some inconsistencies, for example the different simulation volumes probe different environments. As such, this is intended only to provide a qualitative comparison with the observations, with quantitative comparison facilitated by Fig. 7.

Similar to Fig. 7, we see that the EAGLE colours obtained with SKIRT generally show good agreement with the observed distribution. The blue cloud and red sequence populations in EAGLE appear to be in approximately the observed position and contain a roughly similar share of the galaxy population across the mass range. The green valley population is enhanced relative to the T15 photometry, in better agreement with GAMA data. The inconsistent surplus of blue galaxies at the high-mass end, $M_{\star} \gtrsim 10^{10.5} \mathrm{M}_{\odot}$, is also largely suppressed with respect to T15. This is attributable to the more representative treatment of the spatial distribution of the dust in SKIRT, with the ISM dust enshrouding young stars, rather than being distributed in a diffuse galaxy-sized disc as assumed by the screen model of $\mathrm{T} 15$.

However, there are still some notable discrepancies between EAGLE and GAMA. Across all masses the red sequence in EAGLE is flatter than observed, with slightly bluer colours at high mass and redder colours at low mass. This is consistent with the findings of T15, and is symptomatic of the fact that the metallicity of EAGLE galaxies does not increase with $M_{\star}$ as steeply as observed. This is at least in part due to insufficient numerical resolution, as shown by S15 (their fig. 12). A moderate surplus of blue galaxies relative to the observations
Table 3. Maximum likelihood parameters for the model of T15 that best describes the fiducial SKIRT photometry. These values are derived using an MCMC approach, as detailed in Appendix C.

\begin{tabular}{llll}
\hline Model & $\tau_{\text {ISM }}$ & $\tau_{\text {BC }}$ & \multicolumn{1}{c}{$q$} \\
\hline T15 values & 0.33 & 0.67 & 0.2 \\
Ref-100 ML & 0.301 & 0.602 & 0.556
\end{tabular}

can also still be seen between $\sim 10^{10}$ and $\sim 10^{10.5} \mathrm{M}_{\odot}$, likely due to a combination of lower passive fractions and lower typical dust attenuation in the EAGLE galaxies relative to those observed. Differences between the observed and simulated stellar mass functions also contribute to discrepancy: the EAGLE simulation has a deficiency of galaxies at the knee of the mass function $\left(M_{\star} \sim 10^{10.5} \mathrm{M}_{\odot}, \mathrm{S} 15\right)$, such that the contours are skewed to lower masses than in the GAMA distribution.

\subsection{Comparison of SKIRT colours to dust-screen models}

We now turn to comparing the T15 photometry with that generated using SKIRT. The screen model presented by T15 has several parameters, notably $\tau_{\mathrm{BC}}$, the dust optical depth in the birth clouds of stars, $\tau_{\mathrm{ISM}}$, the dust optical depth in the ISM, and $q=b / a$, the axial ratio of the oblate spheroid within which the ISM dust is assumed to be distributed. ${ }^{8}$ The fiducial values of these parameters were informed by observational studies, but they do not necessarily reflect the ISM distribution in EAGLE. To test whether the radiative transfer photometry is better reproduced with a different parametrization of the T15 model, we fit the T15 model to the SKIRT results. The parameter fits are obtained using Bayesian inference, where a Markov-chain Monte Carlo (MCMC) method is used to find the maximum-likelihood parametrization. We simultaneously find the maximum-likelihood (ML) values of $\tau_{\text {ISM }}$ and $q$, enforcing the constraint that $\tau_{\mathrm{BC}}=2 \tau_{\text {ISM }}$ as in the fiducial model of Charlot \& Fall (2000). The application of this constraint and full details of the MCMC procedure are given in Appendix C.

The ML parameters from fitting the fiducial SKIRT model using the free parameters of T15 are given in Table 3. We find that the ML $\tau_{\text {ISM }}$ and $\tau_{\mathrm{BC}}$ values needed to describe the SKIRT results for Ref-100 are $\approx 10$ per cent lower than the fiducial values of $\mathrm{T} 15$. This offset is small, implying similar typical attenuation values in both models. Because the values of $\tau_{\mathrm{ISM}}$ and $\tau_{\mathrm{BC}}$ used in T15 come from the original fitting by Charlot \& Fall (2000) of the SDSS observations, it is encouraging that they are recovered independently by fitting the SKIRT results: this suggests that our SKIRT model yields realistic average optical dust attenuations for galaxies of a given metallicity and gas fraction, and also that the relative stellar and ISM geometries and our dust mapping are reasonable.

However, the $q$ parameter is significantly higher for the SKIRT model, implying less inclination dependence and lower edge-on attenuation in the model, as is indeed seen in Fig. 3. This most likely reflects the artificially 'puffed-up' ISM in simulated galaxies. We repeat that this higher disc thickness is partly set by numerical resolution, but is mostly due to the assumed temperature-density relation for star-forming (disc) gas in the EAGLE subgrid model.

\footnotetext{
${ }^{8}$ Using the standard nomenclature for $a$ and $b$ denoting the primary and secondary axes, respectively. Note that in T15 this is erroneously referred to as $q=a / b$.
} 


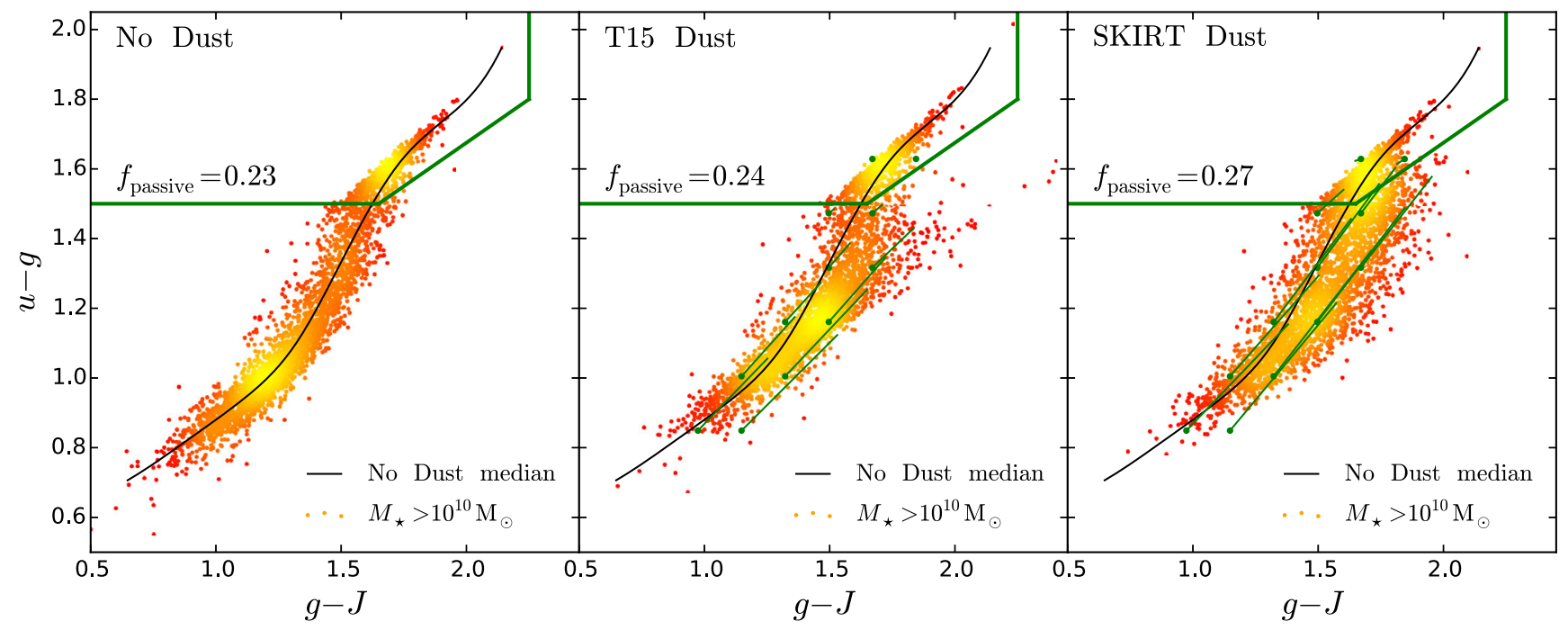

Figure 9. Rest-frame $z=0.1$ ugJ colour-colour diagrams for different photometric models. Points are EAGLE galaxies from simulation Ref-100 with stellar masses $>10^{10} \mathrm{M}_{\odot}$, shaded from red to yellow to indicate local point density. The dust-free photometry of T15, the ML parametrization of the dust-screen model of T15, and the SKIRT photometry including ISM dust and $\mathrm{H}$ II regions are shown in the left, middle and right panels, respectively. Thick, green lines show the passive galaxy cut advocated by Schawinski et al. (2014), the corresponding passive fraction of EAGLE galaxies using this cut is indicated in each panel. Black curves indicate the median $u-g$ values as a function of $g-J$ for the dust-free photometry, these are repeated in each panel to guide the eye. Thin, green lines indicate the mean reddening vectors of EAGLE galaxies in the dusty models relative to the dust-free model. These emanate from green points, specifying the centre of each bin from which a vector is computed. Note that vectors point from bottom-left to top-right. The sKIRT model gives higher passive fractions than the intrinsic and T15 dust photometries, indicating significant pollution of the passive region by star-forming galaxies.

Overall, we find that the fiducial SKIRT model, which is based on physical modelling of the EAGLE galaxies, produces typical dust attenuations at optical to NIR wavelengths similar to the published model of T15. Although the $f_{\text {dust }}$ and $f_{\mathrm{PDR}}$ parameters of the model were chosen to reproduce FIR observables by Camps et al. (2016), this result is in fact independent of that calibration. To demonstrate this, we apply our ML fitting procedure to an 'uncalibrated' SKIRT model, produced using the default literature values of $f_{\text {dust }}$ and $f_{\text {PDR }}$. Similar levels of agreement between the fiducial $\tau_{\mathrm{ISM}}, \tau_{\mathrm{BC}}$ and $q$ values of T15 are recovered, as shown in Appendix D. Given these findings, when comparing models we do not modify the parameters of the SKIRT and screen model from the published values of Camps et al. (2016) and T15. The fiducial SKIRT model photometry and that of the T15 model are compared below.

\subsubsection{Colour-colour distributions}

To further explore the effects of dust reddening, we compare colourcolour distributions for dust-free photometry of EAGLE galaxies from T15, the fiducial dust-screen model of T15 and the full SKIRT modelling in Fig. 9. We plot rest-frame $u-g$ against $g-J$ colours, analogous to the $U V J$ diagram used by Williams et al. (2009), to separate actively star forming but dust-reddened galaxies from intrinsically red and passive galaxies. Data points are shaded from red to yellow by point density, to indicate how galaxies are distributed. Thin green lines indicate the mean reddening vectors for the two dust models relative to the intrinsic photometry of $\mathrm{T} 15$, in regular bins of $u g J$ intrinsic colour. These are only plotted for bins with $>10$ galaxies. The colour-colour bin centres from which the vectors emanate are highlighted by green points. We overplot the $u g J$ cut used to separate active from passive galaxies by Schawinski et al. (2014) in bold green. The passive fractions of EAGLE galaxies, as inferred from applying this cut, are indicated in each panel.
The three photometric models produce qualitatively similar distributions, but with some important differences. The dust-free model in the left-hand panel exhibits two well-defined peaks, a 'blue peak' at $(u-g, g-J) \approx(1.2,1.0)$ and a 'red peak' at $\approx(1.6$, 1.6). The intrinsic distribution is relatively tight for galaxies with $M_{\star}>10^{10} \mathrm{M}_{\odot}$, with $\lesssim 0.2$ mag scatter in $u-g$ for a given $g-J$ colour. The passive fraction is $f_{\text {passive }}=0.23$ for galaxies with $M_{\star}>10^{10} \mathrm{M}_{\odot}$.

The middle panel, showing galaxy colours produced by the fiducial dust-screen model of T15, exhibits a similar distribution in the passive region. From the lack of visible lines at $u-g>1.4$, we see that there is minimal reddening of galaxies into or within this region. The recovered passive fraction of $f_{\text {passive }}=0.24$ reveals that indeed the passive region is $<5$ per cent polluted by the galaxies defined as active in the dust-free (left-hand) panel. The active region galaxies, however, exhibit more variation. While the blue peak is similarly well defined relative to the dust-free colours, the position of the peak is shifted to slightly redder colours by $\sim 0.1 \mathrm{mag}$ and is broadened by scatter to redder colours, with many more star-forming galaxies having $g-J>1.5$. The mean reddening vectors are small relative to the distance between the peak and the most extremely attenuated active galaxies, which have colours $g-J \gtrsim 2$.

The right-hand panel, showing the SKIRT model colours, reveals some consistent features. The passive region galaxies reveal a similar distribution as in the left and middle panels, with a red peak in a very similar position. The active region galaxies also occupy a similar region of the $u g J$ plane to the T15 (middle) panel, with a blue peak shifted to slightly redder colours relative to the dust-free model in the left-hand panel. However, in detail there are some notable differences. The blue peak is significantly depleted relative to the other panels, with the red peak exhibiting a tail to bluer colours. Though the SKIRT model does not possess the extremely reddened galaxies of the T15 (middle) panel, the magnitude of the mean reddening vectors are generally larger across the intrinsic distribution 
and show less reduction with redder intrinsic $u-g$ colour relative to T15. We also see an enhanced 'green' population of galaxies with intermediate colours $1.25<u-g \lesssim 1.5$ in the active region. The enhanced average reddening across the distribution also results in a higher recovered passive fraction of $f_{\text {passive }}=0.27$. This indicates that the passive region has $\mathrm{a} \approx 15$ per cent pollution by galaxies defined as active in the dust-free (left-hand) panel. The reddening of galaxies from the blue peak to the red peak in the $u g J$ diagram corresponds to the significant boost (depletion) of the red (blue) sequence population for the SKIRT photometry relative to the dust-free colours seen in Fig. 7.

The differences between the T15 and SKIRT panels in Fig. 9 can be attributed to the nature of the dust modelling. The $\mathrm{T} 15$ reddening vector is close to parallel with the sloped boundary of the passive region, as illustrated by a tail of extremely reddened active galaxies with $g-J>2$. This is unsurprising, as the screen model of Calzetti et al. (2000) is used by Schawinski et al. (2014) to define the boundary between active and passive galaxies. This may explain why few dusty galaxies move into the passive region when applying the T15 screen model. The SKIRT model, which exploits the 3D distribution of dust around stars, yields generally steeper reddening vectors of higher magnitude, both of which contribute to moving dusty star-forming galaxies into the region were galaxies are deemed to be passive when using the Schawinski et al. (2014) colour-colour cut. This is because nascent stellar populations embedded in dense ISM are effectively shielded in the SKIRT model, leading to more active galaxies masquerading as passive. Again, the fraction of galaxies misclassified as passive could be underestimated due to the lack of highly attenuated edge-on EAGLE galaxies, attributable to the artificially 'puffed-up' ISM in the simulation.

\subsubsection{Passive fractions}

We use the colour-colour cut of Schawinski et al. (2014) from Fig. 9 to calculate passive fractions as a function of mass for both SKIRT and ISM dust-free photometry. The results are shown in Fig. 10, where we compare to the passive fractions calculated directly from the aperture SFRs (see S15; Furlong et al. 2015). The value of the specific star formation rate $\mathrm{SSFR} \equiv \dot{M}_{\star} / M_{\star}$ below which a galaxy is deemed passive is somewhat arbitrary. As we are using the Schawinski et al. (2014) cut in $u g J$ for the colour cut, we use a value of $10^{-2.5} \mathrm{Gyr}^{-1}$ which yields good agreement with the dust-free photometric estimates for numerically well-resolved galaxies $\left(M_{\star} \gtrsim 10^{10} \mathrm{M}_{\odot}\right)$. Note that S15 and Furlong et al. (2015) used a higher value of $10^{-2} \mathrm{Gyr}^{-1}$.

The differences between the photometric passive fraction estimates with and without ISM dust become apparent at masses $M_{\star}>10^{9.5} \mathrm{M}_{\odot}$. For better-resolved galaxies, $M_{\star}>10^{10} \mathrm{M}_{\odot}$, the passive fraction obtained when including dust reddening lies $\approx 0.1$ dex above the value estimated using intrinsic colours or calculated using the sSFR cut. This offset suggests that, using colours alone, $\approx 15$ per cent of the apparently passive population may be misclassified active galaxies for stellar masses $M_{\star}>10^{10} \mathrm{M}_{\odot}$. This fraction could be higher still if our mock photometry had levels of attenuation closer to observation (see Fig. 3). It is important to note that the $u g J$ selection used here also represents a particularly stringent passive cut; hence, why we compare to a sSFR cut of $10^{-2.5} \mathrm{Gyr}^{-1}$ rather than one at $10^{-2} \mathrm{Gyr}^{-1}$. We find that by relaxing the $u g J$ cut (a -0.1 shift in $u-g$ to approximate a $10^{-2} \mathrm{Gyr}^{-1}$ selection) leads to a higher proportion of active galaxies being misclassified as passive due to dust effects $(\approx 38$ per cent). It seems that the use

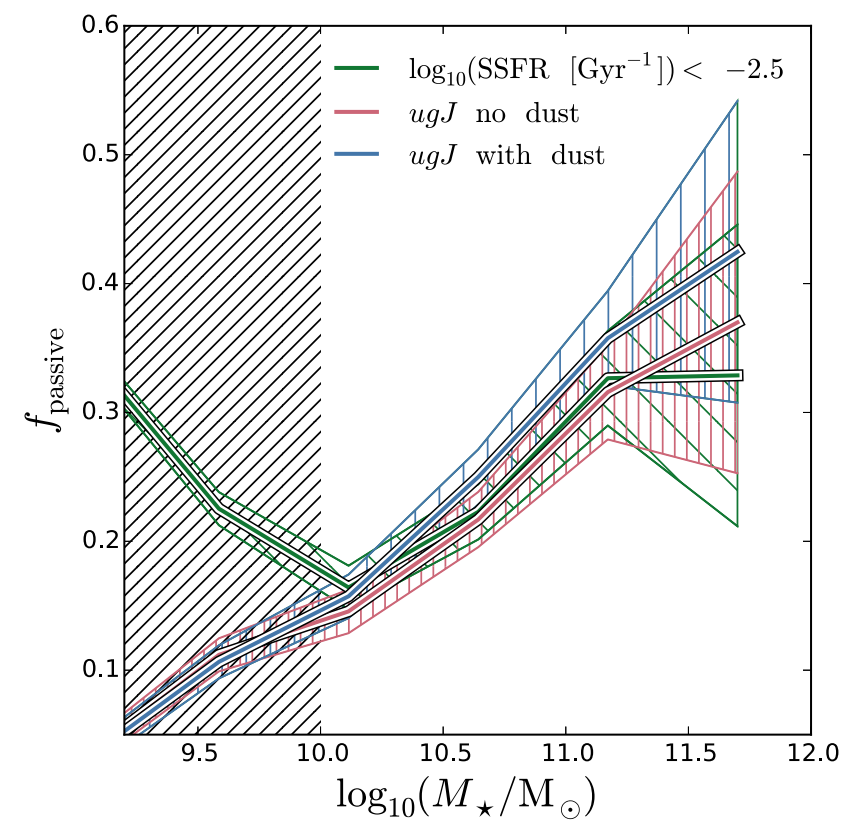

Figure 10. Passive galaxy fractions for the Ref-100 EAGLE simulation in bins of $M_{\star}$, equally spaced in $\log _{10} M_{\star} / \mathrm{M}_{\odot}$. The green line shows the values obtained using a $\dot{M}_{\star} / M_{\star}$ cut at $10^{-2.5} \mathrm{Gyr}^{-1}$. Red and blue lines show the fractions obtained using the photometry without and with ISM dust, respectively. Coloured, hatched regions indicate the uncertainty for each line, corresponding to the fractional Poisson error on the number of galaxies in each bin. The diagonally hatched region $M_{\star}<10^{10} \mathrm{M}_{\odot}$ indicates where the true passive fraction (green line) decreases with stellar mass, due to resolution and volume effects. We see that the discrepancy between the passive fractions obtained using intrinsic and dust attenuated photometry increases with stellar mass above $10^{10} \mathrm{M}_{\odot}$.

of a stringent cut minimizes misclassification of passive galaxies to the 15 per cent level.

There is a striking divergence between the SSFR and photometrically defined passive fractions for galaxies with $M_{\star}<10^{10} \mathrm{M}_{\odot}$ apparent in Fig. 10. This occurs in a region where SFRs are subject to resolution and volume effects; however, it is the metallicities of these galaxies that is likely driving this discrepancy. Indeed, a (passive) 10 Gyr old stellar population with a metallicity of $Z=0.4 \mathrm{Z}_{\odot}$ will lie below the $u-g=1.5$ threshold of Schawinski et al. (2014) in the Bruzual \& Charlot (2003) model - and hence is too blue to be classified as passive. As the SDSS sample of Schawinski et al. (2014) is dominated by galaxies of mass $\gtrsim 10^{10} \mathrm{M}_{\odot}$, it is likely that only a few of the observed galaxies would be affected by this.

\section{SPECTRAL INDICES}

We apply SKIRT modelling to compute two spectral indices that are often used as proxies for star formation activity, the $\mathrm{H} \alpha$ luminosity, $L_{\mathrm{H} \alpha}$ (e.g. Kennicutt 1998), and the strength of the $4000 \AA$ break, D4000 (e.g. Kauffmann et al. 2003a). We compare the indices for EAGLE galaxies to both theoretical and observed values below, concentrating on the effects of dust, and test the correlation of these indices with the intrinsic SFR of EAGLE galaxies.

\subsection{Dust effects on the $\mathrm{H} \alpha$ flux}

The $\mathrm{H} \alpha$ luminosity of a galaxy is thought to be a reliable proxy for its SFR, basically because the recombining gas that emits the 

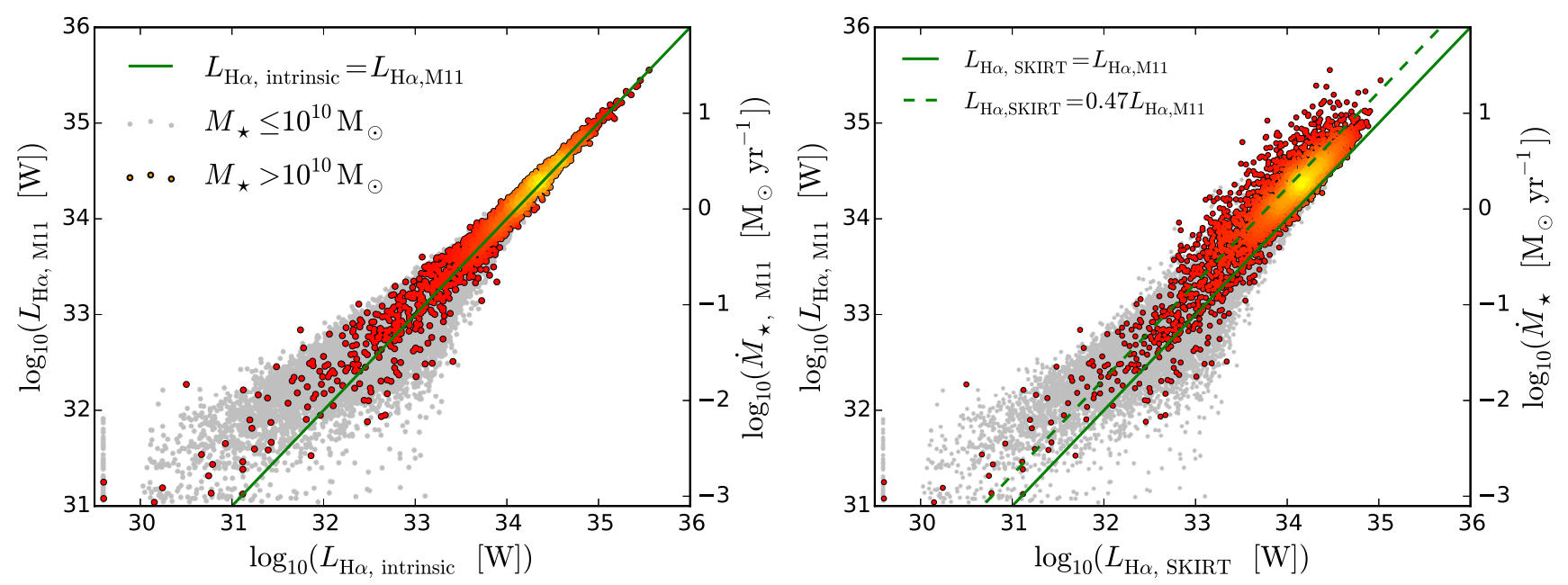

Figure 11. $\mathrm{H} \alpha$ fluxes of EAGLE galaxies. Left-hand panel: ISM dust-free $\mathrm{H} \alpha$ flux from the MAPPINGS-III model compared to $\mathrm{H} \alpha$ flux computed from equation (5), where $\dot{M}_{\star}$ is the instantaneous SFR within a $30 \mathrm{kpc}$ aperture; the value of $\dot{M}_{\star}$ is indicated on the right $y$-axis. Coloured dots are individual EAGLE galaxies with $M_{\star}>10^{10} \mathrm{M}_{\odot}$ with colour a measure of the point density of galaxies in the plot; grey points represent galaxies of lower stellar mass. Galaxies where $\mathrm{H} \alpha$ is not detected are clipped to $\log _{10}\left(L_{\mathrm{H} \alpha}[\mathrm{W}]\right)=29.8$; the green line indicates the 1:1 relation to guide the eye. Right-hand panel: same as left-hand panel, but showing the $\mathrm{H} \alpha$ fluxes computed using SKIRT i.e. including ISM dust; the dashed line is the best linear fit to the coloured points (excluding undetected galaxies), and represents the average dust attenuation factor. $L_{\mathrm{H} \alpha}$ values measured from ISM dust-free spectra generally recover those given by equation (5) very well, as expected, with a relatively spread at low values ascribed to shot noise in the sampling of $\mathrm{H}$ II regions. The dust attenuated values show an offset and large $(\sim 1$ dex $)$ scatter.

$\mathrm{H} \alpha$ photons is thought to be photoionized by massive $\left(>10 \mathrm{M}_{\odot}\right)$ and hence recently (<20 Myr) formed stars (e.g. Kennicutt 1998). However, as we have seen, such star-forming regions are typically dust obscured (not just by the dust in $\mathrm{H}_{\text {II }}$ birth clouds, but by ISM dust as well), and the measured flux therefore needs to be corrected for dust. Here we compare the intrinsic $L_{\mathrm{H} \alpha}$ values, emanating only from $\mathrm{H}$ II regions in our model, to those obtained using empirical corrections to observed spectra. When measuring emission lines, we only use the $\mathrm{H}$ II region component of the source spectra (the blue line in Fig. 1) to minimize the contribution (either in emission or more likely in absorption) from the stellar continuum (a correction that needs to be applied to observational data). This enables us to isolate the effects of dust on inferred line fluxes, which we can investigate with SKIRT, from those caused by continuum fitting.

The relation between $\mathrm{H} \alpha$ line flux and SFR advocated by Murphy et al. (2011) is

$L_{\mathrm{H} \alpha, \mathrm{M} 11}=\left(\frac{\dot{M}_{\star}}{5.37 \times 10^{-42} \mathrm{M}_{\odot} \mathrm{yr}^{-1}}\right) \mathrm{erg} \mathrm{s}^{-1}$,

and is a recalibration of the relation from Kennicutt (1998). The recalibration accounts for the different IMFs assumed by Kennicutt (1998) (Salpeter) compared to Murphy et al. (2011) (Kroupa), and is thus consistent with the (Kroupa) IMF of the MAPPINGS-III models that we use for young stars. For a like-for-like comparison with $L_{\mathrm{H} \alpha}$ values obtained with SKIRT, we use the instantaneous $\dot{M}_{\star}$ within a 30 pkpc aperture from the EAGLE data base (McAlpine et al. 2016). This is calculated by summing over the $\dot{M}_{\star}$ values of gas particles within that aperture.

As a first test of our $\mathrm{H}$ II region prescription and subsequent measurement procedure for calculating $L_{\mathrm{H} \alpha}$, we compare the values obtained using the ISM dust-free spectra, $L_{\mathrm{H} \alpha \text {, intrinsic }}$, to $L_{\mathrm{H} \alpha, \mathrm{M} 11}$ in the left-hand panel of Fig. 11. For this comparison, we overplot galaxies with $\log _{10}\left(M_{\star} / \mathrm{M}_{\odot}\right)>10$ coloured by the local point density, with lower mass galaxies underplotted in grey. The green line indicates the 1:1 relation to guide the eye.
The tight 1:1 correlation between the two values for high SFR, $\dot{M}_{\star} \gtrsim 0.1 \mathrm{M}_{\odot} \mathrm{yr}^{-1}\left(L_{\mathrm{H} \alpha} \gtrsim 10^{33} \mathrm{~W}\right)$, is reassuring, implying that the resampling technique used to parametrize $\mathrm{H}_{\text {II }}$ regions (see Section 3.1.2) reproduces the expected $\mathrm{H} \alpha$ measurements. This good agreement is expected: the same population synthesis models and a similar treatment of nebular components are employed in both the MAPPINGS-III model that we use, and the model used by Murphy et al. (2011) that yields the conversion factor given in equation (5).

At low SFR, the $L_{\mathrm{H} \alpha \text {, intrinsic }} / L_{\mathrm{H} \alpha, \mathrm{M} 11}$ ratio exhibits large scatter. This is due to $\mathrm{H}_{\text {II }}$ regions being sampled stochastically from the mass function of equation (1), yielding increased sampling noise for lower star formation rates. Galaxies without $\mathrm{H}$ II regions, and thus without $\mathrm{H} \alpha$ in our modelling, are plotted at $L_{\mathrm{H} \alpha \text {, intrinsic }}=10^{29.5} \mathrm{~W}$ in the figure.

The right-hand panel shows the corresponding plot for the ISM dust attenuated spectra (without attempting to correct the $\mathrm{H} \alpha$ flux for dust correction). We overplot the $1: 1$ relation with a constant dust attenuation factor of $A=0.47$ (dotted green line, this corresponds to $0.82 \mathrm{mag}$ extinction). This factor is the mean offset between the measured (dust attenuated) and intrinsic $\mathrm{H} \alpha$ luminosities, as determined using a least-squares fit for Ref-100 galaxies of $\log _{10}\left(M_{\star} / \mathrm{M}_{\odot}\right)>10$. The convergence of $\mathrm{H} \alpha$ luminosities and $A$ values are tested for the other simulations listed in Table 1 in Appendix B2. The observed average attenuation in local galaxies is $\approx 0.4$ (Kennicutt 1992 ) ( $1 \mathrm{mag}$ of extinction), a factor of $\approx 1.2$ lower, but our value is still within the systematic uncertainty of the average extinction inferred from Balmer decrement measurements at $z \lesssim 0.5$ (e.g. Ly et al. 2012).

\subsection{The $\mathrm{H} \alpha$ luminosity function}

Having shown that our implementation of mock $\mathrm{H} \alpha$ emission lines yields line luminosities consistent with the underlying assumed SSP model, we now proceed to compute the $\mathrm{H} \alpha$ luminosity function and compare it to data. It is common practise to apply a constant dust correction to observed $\mathrm{H} \alpha$ fluxes obtained from narrow-band 
surveys to infer 'intrinsic', ISM dust-free, luminosities (e.g. Sobral et al. 2013). This yields an (intrinsic) $\mathrm{H} \alpha$ line luminosity function with a Schechter form. However, the bright-end slope of the bestfitting function that results from applying a constant dust correction is steeper than when the dust correction is performed using Balmer decrements (e.g. Gilbank et al. 2010; Gunawardhana et al. 2013). This systematic difference is partially attributable to the SFR dependence of attenuation ${ }^{9}$ (Brinchmann et al. 2004; Zahid et al. 2013). Because SKIRT provides both the ISM dust-free and dust-attenuated SEDs, we can compare with the 'true' dust correction for our simulated galaxies at different orientations and investigate this effect further.

A potential caveat for the realism of $\mathrm{H} \alpha$ luminosities computed for EAGLE galaxies is that the simulated specific SFRs may be low compared to observations, both locally and at higher redshifts (Furlong et al. 2015). This is in fact somewhat puzzling since the stellar mass functions do agree relatively well. The observed SFRs are inferred from various proxies, including emission lines, FIR and radio data. However, the calibration may rely on assumptions about the UV continuum, which depends on the assumed IMF and population synthesis model (see Kennicutt 1998). Some recent studies suggest that stellar rotation and binary stars may contribute more to the UV continuum than previously thought, affecting this calibration (e.g. Hernández-Pérez \& Bruzual 2013; Horiuchi et al. 2013). When instead considering the $\mathrm{H} \alpha$ fluxes (including dust effects) the realism of galaxy attenuation and ISM also becomes important. Discrepancies in SFRs and ISM attenuation can have degenerate effects on the $\mathrm{H} \alpha$ measurements. It is therefore important to consider the intrinsic $\mathrm{H} \alpha$ and dust-attenuated $\mathrm{H} \alpha$ measurements separately to isolate reasons for discrepancy or agreement.

Recently, Chang et al. (2015) performed SED fitting of galaxies with SDSS and WISE photometry and obtained SFRs for $z \approx 0.1$ star-forming galaxies. Their values are systematically lower by $\approx 0.2$ dex compared to previous work such as that of Kennicutt (1998), and in better agreement with those predicted by EAGLE. If the lower $\dot{M}_{\star}$ values of Chang et al. (2015) point to a previous miscalibration of SFR indicators, then the $L_{\mathrm{H} \alpha}$ values predicted by equation (5), and thus present in the intrinsic EAGLE SEDs (see Fig. 11), may be too low. To test the effect of such a change in normalization when comparing to the $\mathrm{H} \alpha$ luminosity function, we plot $L_{\mathrm{H} \alpha}$ both with and without a +0.2 dex shift, referring to this as the Chang et al. (2015) conversion. ${ }^{10}$

Fig. 12 compares mock $\mathrm{H} \alpha$ luminosity functions to data. Comparing thick and thin green lines, which use the same $\dot{M}_{\star}$ to $\mathrm{H} \alpha$ flux conversion, but correspond to imposing a mass limit of $M_{\star} \geq$ $2.26 \times 10^{7} \mathrm{M}_{\odot}(100$ star particles at high resolution $)$ and $M_{\star} \geq$ $1.81 \times 10^{8} \mathrm{M}_{\odot}$, respectively, enables us to estimate the level of numerical convergence. A hatched region marks where these differ by more than 10 per cent, i.e. where incompleteness effects become important for the higher mass cut. We therefore focus on the model $\mathrm{H} \alpha$ luminosities above $10^{33} \mathrm{~W}$.

Up to luminosities of $\sim 10^{34} \mathrm{~W}$, the solid green and solid blue curves (that both use equation 5 ) differ by $\approx 0.5 \mathrm{dex}$ (a factor of three), indicating that the Recal-25 simulation predicts significantly higher values of $\phi$ then Ref-100, which is in better agreement with

\footnotetext{
${ }^{9}$ Poor sampling of the strongest $\mathrm{H} \alpha$ emitters, due to the small volume of narrow-band surveys, may also contribute a steep bright-end.

${ }^{10}$ Note that Chang et al. (2015) do not explicitly advocate such a correction, rather, this conversion represents the case that the discrepancy they find exists due to previous miscalibration of absolute SFR.
}

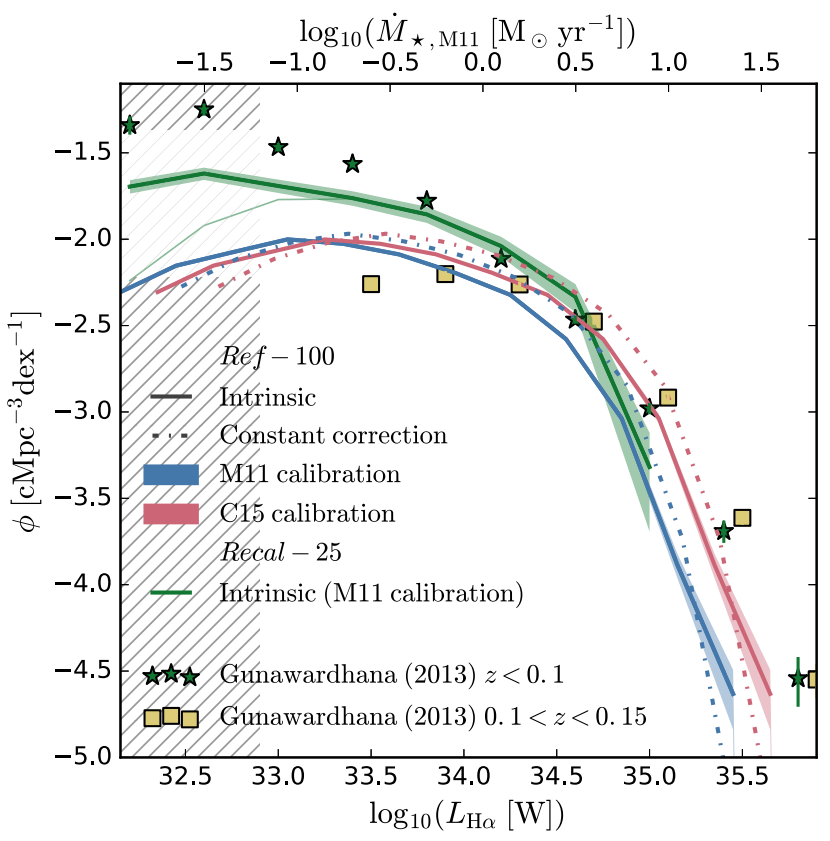

Figure 12. Redshift $z=0.1$ dust-corrected $\mathrm{H} \alpha$ luminosity functions of EAGLE galaxies compared to observations. Solid coloured lines are the ISM dust-free EAGLE LFs for simulation Ref-100 for randomly oriented EAGLE galaxies with $M_{\star} \geq 1.81 \times 10^{8} \mathrm{M}_{\odot}$ using the Murphy et al. (2011) and Chang et al. (2015, see text) ratio of $M_{\star} / L_{\mathrm{H} \alpha}$, respectively, with the shading showing the Poisson error range. The dot-dashed curves are the corresponding LFs computed using SKIRT, dust corrected using the best-fitting constant dust correction to the attenuated spectra ( $A$, see Fig. 11). Green curves are the ISM dust-free LF for simulation Recal-25 using the Murphy et al. (2011) ratio of $\dot{M}_{\star} / L_{\mathrm{H} \alpha}$, for galaxies with $M_{\star} \geq 1.81 \times 10^{8} \mathrm{M}_{\odot}$ and $M_{\star}$ $\geq 2.26 \times 10^{7} \mathrm{M}_{\odot}$ shown as thin and thick green lines, respectively. The hatched region marks where these deviate by $>10$ per cent, indicating luminosities for which incompleteness is important for the $M_{\star} \geq 1.81 \times 10^{8}$ mass limit. Observational data are taken from Gunawardhana et al. (2013), who use the Balmer decrement dust-corrected GAMA sample, for redshift ranges $z<0.1$ (star symbols) and $0.1<z<0.15$ (squares). Error bars indicate the statistical uncertainty on observed data points, but are too small to be seen for all but the highest luminosities. The simulated LF using the Chang et al. (2015) calibration is closer to the observed measurements; the intrinsic LFs possess a broader knee than when a constant dust correction is applied.

the data. As seen in Fig. 11, below $L_{\mathrm{H} \alpha} \sim 10^{34} \mathrm{~W}$ the poor sampling of $\mathrm{H}_{\text {II }}$ regions contributes to this resolution effect. For the hatched region, $L_{\mathrm{H} \alpha} \lesssim 10^{32.9} \mathrm{~W}$, the discrepancy is driven by incompleteness due the imposed mass limit for the Ref-100 simulation. However, at intermediate luminosities this is due to real differences between the properties of the galaxies in the high- and standard-resolution runs. Indeed, we recall from Fig. 7 that these two simulations differ substantially in the lowest mass bin: Recal-25 galaxies tend to be intrinsically blue and star forming, whereas a significant fraction of Ref-100 galaxies are intrinsically red and passive. This contributes to the boost in the Recal-25 luminosity function at intermediate $L_{\mathrm{H} \alpha}\left(10^{32.9} \lesssim L_{\mathrm{H} \alpha} \lesssim 10^{34} \mathrm{~W}\right)$, yielding better agreement with the observed luminosity functions. The higher number density of galaxies at these intermediate $\mathrm{H} \alpha$ luminosities is due to the similar contribution of volume and resolution effects. Volume effects and convergence are discussed further in Appendix B2. The offset between the blue and green curves at lower luminosities is therefore, in part, a measure of numerical convergence.

We next compare solid lines (intrinsic luminosities) to dot-dashed lines (SKIRT dust-attenuated luminosities corrected using a constant 
dust correction), for either blue or red lines. These agree well at the faint end $\left(L_{\mathrm{H} \alpha} \lesssim 10^{34} \mathrm{~W}\right)$ where the luminosity functions are close to flat, but significant differences can be seen for brighter galaxies, with the number of bright sources higher at the knee of the constant dust correction luminosity function. A constant dust correction tends to overestimate the true level of dust attenuation around the knee, and underpredict it for the most $\mathrm{H} \alpha$ bright galaxies, resulting in a steeper bright end slope. Note that the constant dust correction we use is essentially indistinguishable from the common observational assumption of 1 mag in this plot.

Finally, we compare the intrinsic $\mathrm{H} \alpha$ luminosity function (solid lines) to the observed result corrected for dust using the Balmer decrement (symbols). Although similar at faint fluxes, $L_{\mathrm{H} \alpha} \lesssim 10^{35}$ $\mathrm{W}$, the simulated luminosity function is significantly below the observations at the bright-end when using equation (5) (blue), with the difference much reduced when using the Chang et al. (2015) conversion (red curve). The shape at the bright-end is so steep that even a small error in the observed luminosity determination can make EAGLE and GAMA consistent. In addition, the EAGLE stellar mass function is lower than observed around the knee (S15), which will also contribute to the deficit at brighter luminosities.

In summary, the EAGLE $\mathrm{H} \alpha$ luminosity function is in relatively good agreement with observations when applying the Chang et al. (2015) inspired ${ }^{7}$ conversion between $\dot{M}_{\star}$ and $\mathrm{H} \alpha$ luminosity. The effects of insufficient numerical resolution are apparent at lower luminosities $\left(L_{\mathrm{H} \alpha} \lesssim 10^{34} \mathrm{~W}\right)$. Applying a constant correction to the EAGLE dust-attenuated $\mathrm{H} \alpha$ values does not reproduce the shape of the underlying 'true' EAGLE $\mathrm{H} \alpha$ luminosity function well, overestimating the SFRs at higher $\mathrm{H} \alpha$ luminosities $\left(L_{\mathrm{H} \alpha} \gtrsim 10^{34} \mathrm{~W}\right)$. This comes about due to the higher attenuation in more $\mathrm{H} \alpha$ luminous galaxies, shown directly in Appendix B2.

\subsection{D4000 index}

The $4000 \AA$ break (D4000) was used by Kauffmann et al. (2003a) to analyse a large sample of galaxies from the SDSS. In older stellar populations, the opacity due to several ions in stellar atmospheres combines to induce a noticeable discontinuity in the flux around $4000 \AA$, which is mostly absent in hot stars. The size of the discontinuity is hence a measure of the relative contribution of young hot stars to the flux - and hence of the sSFR of the galaxy (e.g. Bruzual 1983). The D4000 index is the logarithm of the ratio of the red and the blue continuum, measured using narrow-band top-hat filters. We use [4050, 4250] $\AA$ and $[3750,3950] \AA$ to sample the red and blue continuum, respectively, as defined by Bruzual (1983). The D4000 index is commonly used to distinguish between active (star-forming) and passive populations, similar to the colour-colour plots of Fig. 9. Indeed, D4000 can be thought of as a colour index (analogous to e.g. $u-r$ ), but is generally considered robust against dust effects due to it being a differential measure with a relatively small wavelength separation between bands.

The D4000 distribution used by Kauffmann et al. (2003a,b) are from a flux-limited selection of SDSS galaxies, and shows strong bi-modality where massive galaxies have a high value of D4000, low-mass galaxies have low values, with the transition mass around $M_{\star} \sim 3 \times 10^{10} \mathrm{M}_{\odot}$, see fig. 1 of Kauffmann et al. (2003b). Even the largest, $100^{3} \mathrm{Mpc}^{3}$, EAGLE volume does not have enough massive galaxies to allow for a direct comparison with these measurements.

To make a comparison to data meaningful, we therefore create a sample of SDSS galaxies mass matched to the EAGLE population over the range $10^{10} \mathrm{M}_{\odot}<M_{\star}<10^{11} \mathrm{M}_{\odot}$, using the observed masses from Kauffmann et al. (2003a). The corresponding

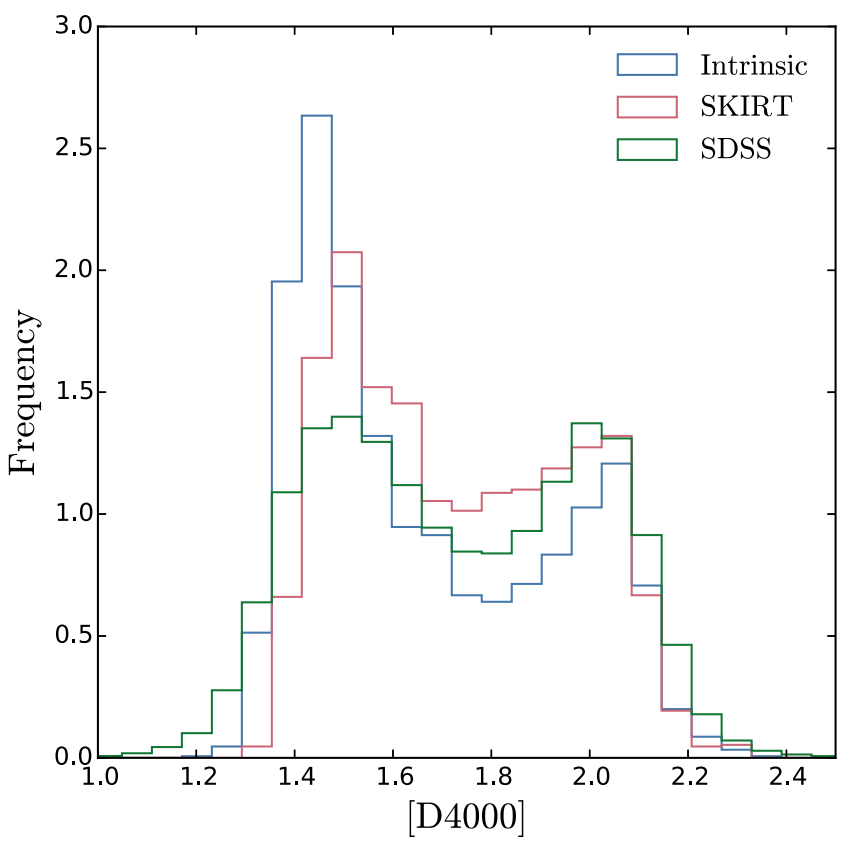

Figure 13. Histogram of D4000 values for intrinsic and radiative transfer (SKIRT) photometry of $z=0.1$ galaxies with $10^{10} \mathrm{M}_{\odot}<M_{\star}<10^{11} \mathrm{M}_{\odot}$. The low and high D4000 peaks are taken to represent active and passive populations, respectively. Blue indicates the intrinsic values, red after dust is applied. Values of D4000 measured for a mass-matched sample of SDSS galaxies are plotted in green. A considerable difference in the active and passive peaks recovered with this technique is observed when dust is applied.

broad-band (Bruzual 1983) D4000 values are taken from the MPAJHU catalogue, released for SDSS DR7 (Abazajian et al. 2009). We then compare the D4000 distribution to that of EAGLE for both the intrinsic and dust-reddened spectra in Fig. 13. Each distribution is normalized to integrate to unity. We employ a cut at D4000 $=1.8$ to separate the active and passive population, which is near the minima of each histogram. We recover passive fractions of 32 per cent and 41 per cent for EAGLE galaxies using intrinsic and SKIRT spectra, as compared to 55 per cent for the SDSS sample.

We first compare the 'intrinsic', ISM dust-free, EAGLE distribution (blue) to that produced using SKIRT (red). Both distributions exhibit a clear bi-modality, with low and high D4000 peaks at $\approx 1.5$ and $\approx 2$, respectively. A stark difference between the two distributions is that the population with low D4000, a common proxy for the star formation, is significantly depleted when ISM dust is included with SKIRT. There is also a shift of the low D4000 peak to higher values. The relative depletion of the low D4000 population in the SKIRT distribution corresponds to a relative boost at intermediate and high D4000. This boost reduces in significance for higher D4000 values, falling below 10 per cent near the high D4000 peak of $\approx 2$. Although there is little difference in overall attenuation between the two D4000 bands, including the preferential obscuration of light from young stars by dust attributed both stellar birth clouds and the diffuse ISM via SKIRT leads to some star-forming galaxies registering higher D4000 values, and even appearing completely passive in this proxy. This is the same effect seen for broad-band colours in Figs 7 and 9 . We find that $\approx 20$ per cent of EAGLE galaxies deemed to be passive according to the $\mathrm{D} 4000>1.8$ criterion, are star forming.

We next compare the EAGLE distributions to the SDSS distribution (green). A clear bi-modality can be seen in the SDSS distribution, exhibiting similar peak positions to EAGLE at D4000 $\approx 1.5$ and $\approx 2$. The SDSS peak positions agree more closely with the SKIRT model 
distribution, which should be a fairer comparison. The fraction of galaxies in the low D4000 (active) population for the SDSS sample is smaller than for either EAGLE distributions, but agrees better with the SKIRT histogram. The high D4000 (passive) fraction is larger for SDSS than EAGLE, and also closer to the dust-reddened values of SKIRT. The inferred passive fraction for SDSS galaxies is 70 per cent higher than inferred for the intrinsic EAGLE spectra, and 35 per cent higher than for SKIRT. While the frequency of SDSS galaxies at intermediate $\mathrm{D} 4000$ values $(\approx 1.8)$ is underpredicted by the intrinsic EAGLE distribution, the boost in the population seen for the SKIRT distribution overpredicts the number density of galaxies by a similar factor. Generally the agreement with observation is improved by the inclusion of SKIRT ISM dust modelling, but remains slightly discrepant. While modelled dust effects can improve the inferred passive fractions, an excess of high-mass active galaxies persists relative to observation. This could reflect a genuine overproduction of active galaxies in EAGLE, as suggested by Furlong et al. (2015).

Additionally we see that the SDSS distribution is broader than for EAGLE, with tails extending to more extreme high and low values. These tails might be due to outliers with unusually large photometric errors. The limited parameter coverage of the populations synthesis models could also prevent the occurrence of the most extreme values in the simulation.

We used the D4000 continuum band definitions of Bruzual (1983) rather than the narrower band definition of Balogh et al. (1999) employed by Kauffmann et al. (2003a). The reason for this choice is that D4000 is better converged at our standard spectral resolution for SKIRT. A caveat is that the broad-band definition is observed to be significantly more susceptible to dust effects than narrow bands (Balogh et al. 1999; Kauffmann et al. 2003a), potentially leading to larger dust uncertainties.

To test how well the measured D4000 predicts star formation activity for the EAGLE spectra, we plot D4000 against sSFR for $z=0.1$ 'active' $\left(\dot{M}_{\star}>0\right)$ EAGLE galaxies in Fig. 14. At $\log _{10}\left(\mathrm{sSFR} / \mathrm{yr}^{-1}\right)>-12$, and despite a clear negative trend of D4000 with sSFR, we see that the median D4000 is higher for the SKIRT spectra (red circles) than for the spectra without ISM dust (blue circles). The distribution of active galaxies in this plane also shows a significant tail to high D4000 when ISM dust is included (shaded squares), a significant number of galaxies with $\log _{10}\left(\mathrm{sSFR} / \mathrm{yr}^{-1}\right)>-11.5$ have $\mathrm{D} 4000$ consistent with the passive population. This suggests that dust effects are significant for this index when applied to the SKIRT spectra, and can be ascribed to star formation being hidden by the dense, dusty ISM of some galaxies. Conversely, a few outlying galaxies (red points) show relatively low D4000 at $\log _{10}\left(\mathrm{sSFR} / \mathrm{yr}^{-1}\right)<-12$. This is due to these galaxies being relatively metal poor, analogous to the anomalously blue passive galaxies identified in Trayford et al. (2016) using $u-r$ colour (their fig. 1d).

\section{SUMMARY AND CONCLUSION}

We have made mock optical observations of galaxies simulated within the EAGLE suite of cosmological, hydrodynamical simulations, including the effects of dust, utilizing the public SKIRT ${ }^{11}$ code. SKIRT calculates three-dimensional radiative transfer on each galaxy, and we used the cool, enriched gas as a tracer of dust in the diffuse ISM. To ameliorate limitations resulting from limited numerical resolution, we apply a subgrid model to represent unresolved $\mathrm{H}_{\text {II }}$

${ }^{11}$ www.skirt.ugent.be
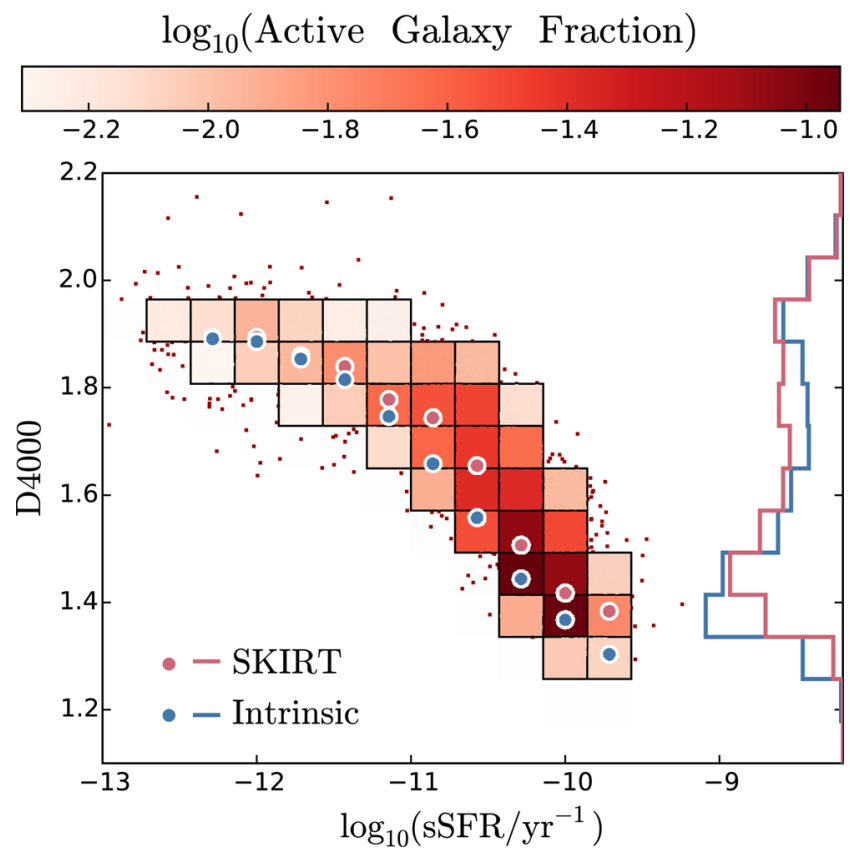

Figure 14. D4000 index as a function of sSFR for $z=0.1$ EAGLE galaxies with $M_{\star}>10^{10} \mathrm{M}_{\odot}$. Circles indicate the median D4000 value in bins of $\log _{10} \mathrm{SSFR}$, with blue representing the intrinsic values and red including dust (SKIRT). Shaded squares represent evenly spaced bins in D4000 and $\log$ sSFR. These are shaded by the fraction of active galaxies (SFR $>0$ ) they contain for the SKIRT values of D4000, and are only displayed within the 95 per cent contour of the active galaxy distribution. Galaxies outlying the 95 per cent contour are represented individually as red points. D4000 histograms are also displayed as in 13, again blue represents intrinsic values and red SKIRT values. We see that, despite the trend of D4000 with SSFR for $\log _{10}\left(\mathrm{SSFR} / \mathrm{yr}^{-1}\right)>-12$, a significant number of galaxies are scattered towards the high D4000 population when dust is included. A few galaxies also exhibit relatively low D4000 despite low sSFRs $\left[\log _{10}\left(\mathrm{SSFR} / \mathrm{yr}^{-1}\right)<-12\right]$.

regions and the associated dust attenuation by using the MAPPINGS-III model of Groves et al. (2008). The full procedure is presented in Section 3, and was developed in this work and in the companion work of Camps et al. (2016, hereafter C16). The dust modelling introduces extra parameters, such as the dust-to-gas ratio in the ISM, and parameters of the MAPPINGS-III model such as the covering factor and compactness of $\mathrm{H}$ II regions. These were chosen by comparing far-infrared mock observations of EAGLE galaxies with observations of local galaxies, as described by Camps et al. (2016). We apply no additional calibration in the optical regime.

To enable a detailed comparison of numerically resolved EAGLE galaxies to observations from the GAMA survey (Driver et al. 2011), we selected galaxies at redshift $z=0.1$ above a stellar mass cut of $M_{\star} \geq 1.81 \times 10^{8} \mathrm{M}_{\odot}(\sim 100$ star particles at standard resolution $)$. We focus on the largest fiducial EAGLE simulation, a $100^{3} \mathrm{Mpc}^{3}$ volume, which provides a sample of 30145 galaxies that satisfy this selection criterion. Galaxies are defined and selected in the same way as in Trayford et al. (2015), to enable direct comparison with their dust-screen model. Integrated spectra, broad-band magnitudes, broad-band images and mock IFU data were produced for each galaxy for three orientations (face-on, edge-on and along the $z$-axis of the simulation volume), with properties detailed in Section 3.3. These will be made available through the public EAGLE data base (McAlpine et al. 2016). 
In Section 4, we studied the attenuation in the $B$-band as a function of inclination, comparing to the observationally inferred relation of Driver et al. (2007). We found that

(i) The $B$-band attenuation-inclination relation for EAGLE galaxies, Fig. 3, exhibits large galaxy-to-galaxy scatter, on top of a smooth trend of increasing median attenuation for galaxies seen more edgeon. This trend is weaker than observed, as is the level of the edge-on attenuation. We attribute this to the fact that the stellar and gas disc in EAGLE galaxies is thicker than in observed galaxies, a consequence of the artificial pressure floor imposed on the simulated ISM (as opposed to being purely limited by numerical resolution, see Fig. 4).

(ii) Stacked attenuation curves of EAGLE galaxies at different orientations, Fig. 6, reveal different profiles. Face-on galaxies show the steepest frequency dependence as the youngest stars are preferentially dust obscured by the diffuse ISM component. Conversely, the curve for edge-on galaxies shows a weaker (or 'greyer') frequency dependence as both young and old stellar populations are obscured by the diffuse dust disc, closer to a screen model. We also see that nebular emission features ( $\operatorname{such}$ as $\mathrm{H} \alpha$ ) suffer from strongly increased ISM attenuation (over and above the attenuation of the stellar birth clouds), due to star-forming regions being embedded in denser and dusty gas.

Comparing optical SKIRT photometry to ISM dust-free models, the dust-screen model of Trayford et al. (2015), and colours from the GAMA survey, we find that

(i) Optical SKIRT galaxy colours match the data remarkably well, Fig. 7. In fact, they match significantly better than the dust-screen model galaxies of Trayford et al. (2015). The SKIRT colours exhibit a mass-dependent boost of the 'green valley' and red populations compared to either intrinsic or dust-screen colours.

(ii) The improved agreement with observation demonstrated by SKIRT, relative to the screen model of Trayford et al. (2015), is attributable to the spatial distribution of dust compared to that of young stars, and the better capturing of orientation effects as compared to a screen model. Fig. 5 shows how highly inclined galaxies with intrinsically blue colours may scatter to the reddest colours.

(iii) Dusty galaxies can confound simple star formation activity proxies, such as colour-colour cuts. Fig. 9 shows that a $u g J$ colourcolour cut can recover passive fractions well when dust is modelled as a screen, but with the more realistic SKIRT dust modelling a significant fraction of active galaxies masquerades as passive. We find that for the relatively stringent cut used in this paper, approximately 15 per cent of galaxies with $M^{\star}>10^{10} \mathrm{M}_{\odot}$ are classified as passive due to dust reddening. More relaxed cuts may yield significantly higher misclassification rates (see Section 5.2.2 for details).

Finally, we investigated spectral indices often used as proxies for star formation activity, such as the $\mathrm{H} \alpha$ line flux and the $4000 \AA$ break (D4000). We plotted the $\mathrm{H} \alpha$ luminosity functions $(\phi)$ and compared directly to the function computed for the GAMA sample by Gunawardhana et al. (2013) in Fig. 12. Given that the star formation rates of EAGLE galaxies are typically 0.2 dex lower than reported for observations using the Kennicutt (1998) calibration (Furlong et al. 2015), and that the conversion from $\dot{M}^{\star}$ to $\mathrm{H} \alpha$ follows the standard Kennicutt (1998) relation (see Fig. 11), it is unsurprising that the EAGLE luminosity function is systematically low at the bright end. However, the recent study of Chang et al. (2015) argued that absolute $\dot{M}^{\star}$ values should be normalized $\approx 0.2$ dex lower, so we also plotted $\phi$ using $\mathrm{H} \alpha$ boosted by this factor. The D4000 distributions were compared to a mass-matched sample of SDSS galaxies in the range
$10^{10} \mathrm{M}_{\odot}<M^{\star}<10^{11} \mathrm{M}_{\odot}$. From studying these indices, we found that

(i) The bright end of the recalibrated, intrinsic $\mathrm{H} \alpha$ luminosity function $\left(L_{\mathrm{H} \alpha}>10^{34.5} \mathrm{~W}\right)$ agrees reasonably with observations, particularly when using the Chang et al. (2015) calibration. Applying a constant dust correction to the SKIRT $\mathrm{H} \alpha$ predictions distorts the shape of the luminosity function, appearing more Schechter-like with a steeper bright end than seen in either the intrinsic or observed LFs.

(ii) D4000 values produced by SKIRT show similar distributions to a mass-matched SDSS sample over the range $10^{10} \mathrm{M}_{\odot}<M_{\star}<10^{11} \mathrm{M}_{\odot}$, Fig. 13, but with somewhat fewer galaxies at high D4000. Dust reddening significantly boosts the high D4000 population relative to the intrinsic distribution, analogous to the red sequence boosting observed in Fig. 7. This leads to higher inferred passive fractions, with the light from young stars preferentially extinguished by dust. The scattering of galaxies to high D4000 by dust, for a given sSFR, is characterized in Fig. 14.

Including dust radiative transfer effects with SKIRT allows us to model the inhomogeneous dust distribution and how that is correlated with regions of recent star formation, while improving the level of agreement of mock fluxes with observations compared to the dust-screen model of Trayford et al. (2015). However, there are clear limitations. In particular, the edge-on attenuation of disc galaxies is lower than observed. Improving this would likely require higher resolution simulations with an explicit cold phase, allowing us to resolve thin molecular gas discs on scales $\lesssim 100 \mathrm{pc}$.

It is perhaps surprising that despite these limitations, the attenuation computed using SKIRT profoundly influences colours, improving agreement with data. This can be ascribed to the effects that geometry and scattering have on the optical attenuation of galaxies that cannot be captured by screen models. The localized nature of the dusty ISM around young stars is effective at hiding their blue light, leaving the older populations to contribute relatively more to the fluxes. Despite invoking similar mean attenuation values (see Section 5.2, Appendix D), a screen model yields more apparently blue galaxies because their star-forming regions are relatively much less shielded by dust.

Throughout this paper, we focus on using a forward modelling approach to compare simulated and real galaxies in the observable domain. While we discuss many benefits of this approach, we note that forward modelling has the potential to obscure the reasons for discrepancy (or agreement) if the influence of individual physical properties are not well understood. An example where such caution is needed would be in our $\mathrm{H} \alpha$ measurements, influenced by both the ISM structure and star formation rates of EAGLE galaxies. We emphasize that it is therefore important to analyse our forward modelling results in the context of comparison studies in the physical domain (such as Furlong et al. 2015; Schaye et al. 2015, for EAGLE galaxies). We have attempted to provide such context in this work.

We hope that the more realistic observables produced by SKIRT provide a useful resource, opening new avenues of investigation for comparing observations to the simulations. Mock observational data presented in this paper will be made accessible via the public EAGLE data base (McAlpine et al. 2016, for updates register at http://icc.dur.ac.uk/Eagle/database.php). Our mock observables are also provided for additional redshifts and EAGLE simulations that are not discussed in this work. 


\section{ACKNOWLEDGEMENTS}

JWT would like to acknowledge Peder Norberg for insightful comments at the early stages of this project. We also thank the anonymous referee, whose comments and suggestions lead to a significant improvement of the manuscript. The work was supported by the Science and Technology Facilities Council [grant number ST/F001166/1], by the Interuniversity Attraction Poles Programme initiated by the Belgian Science Policy Office (AP P7/08 CHARM) by ERC grant agreement 278594 - GasAroundGalaxies and by Netherlands Organisation for Scientific Research (NWO), through VICI grant 639.043.409. We used the DiRAC Data Centric system at Durham University, operated by the Institute for Computational Cosmology on behalf of the STFC DiRAC HPC Facility (www.dirac.ac.uk). This equipment was funded by BIS National E-Infrastructure capital grant ST/K00042X/1, STFC capital grant ST/H008519/1 and STFC DiRAC is part of the National E-Infrastructure. RAC is a Royal Society University Research Fellow. The data used in the work is available through collaboration with the authors. GAMA is a joint European-Australasian project based around a spectroscopic campaign using the Anglo-Australian Telescope. The GAMA input catalogue is based on data taken from the Sloan Digital Sky Survey and the UKIRT Infrared Deep Sky Survey. Complementary imaging of the GAMA regions is being obtained by a number of independent survey programmes including GALEX MIS, VST KiDS, VISTA VIKING, WISE, HerschelATLAS, GMRT and ASKAP providing UV to radio coverage. GAMA is funded by the STFC (UK), the ARC (Australia), the AAO and the participating institutions. The GAMA website is http://www.gama-survey.org/.

\section{REFERENCES}

Abazajian K. N. et al., 2009, ApJS, 182, 543

Altay G., Theuns T., 2013, MNRAS, 434, 748

Aoyama S., Hou K.-C., Shimizu I., Hirashita H., Todoroki K., Choi J.-H., Nagamine K., 2017, MNRAS, 466, 105

Baes M., Camps P., 2015, Astron. Comput., 12, 33

Baes M., Dejonghe H., 2001, MNRAS, 326, 733

Baes M. et al., 2003, MNRAS, 343, 1081

Baes M., Verstappen J., De Looze I., Fritz J., Saftly W., Vidal Pérez E., Stalevski M., Valcke S., 2011, ApJS, 196, 22

Bahé Y. M. et al., 2016, MNRAS, 456, 1115

Baldry I. K. et al., 2007, MNRAS, 380, 963

Baldry I. K. et al., 2014, MNRAS, 441, 2440

Baldwin J. A., Phillips M. M., Terlevich R., 1981, PASP, 93, 5

Balogh M. L., Morris S. L., Yee H. K. C., Carlberg R. G., Ellingson E., 1999, ApJ, 527, 54

Bekki K., 2015, ApJ, 799, 166

Best P. et al., 2013, Astrophys. Space Sci. Proc. Vol. 37, Thirty Years of Astronomical Discovery with UKIRT. Springer-Verlag, Berlin, p. 235

Bourne N. et al., 2013, MNRAS, 436, 479

Brinchmann J., Charlot S., White S. D. M., Tremonti C., Kauffmann G., Heckman T., Brinkmann J., 2004, MNRAS, 351, 1151

Brinks E., Burton W. B., 1984, A\&A, 141, 195

Bruzual A. G., 1983, ApJ, 273, 105

Bruzual G., Charlot S., 2003, MNRAS, 344, 1000

Byun Y. I., Freeman K. C., Kylafis N. D., 1994, ApJ, 432, 114

Calzetti D., 2001, New Astron. Rev., 45, 601

Calzetti D., Armus L., Bohlin R. C., Kinney A. L., Koornneef J., StorchiBergmann T., 2000, ApJ, 533, 682

Camps P., Baes M., 2015, Astron. Comput., 9, 20

Camps P., Trayford J. W., Baes M., Theuns T., Schaller M., Schaye J., 2016, MNRAS, 462, 1057 (C16)

Chabrier G., 2003, PASP, 115, 763
Chang Y.-Y., van der Wel A., da Cunha E., Rix H.-W., 2015, ApJS, 219, 8

Charlot S., Fall S. M., 2000, ApJ, 539, 718

Conroy C., Gunn J. E., White M., 2009, ApJ, 699, 486

Crain R. A. et al., 2015, MNRAS, 450, 1937 (C15)

De Cia A., Ledoux C., Savaglio S., Schady P., Vreeswijk P. M., 2013, A\&A, 560, A88

De Geyter G., Baes M., Camps P., Fritz J., De Looze I., Hughes T. M., Viaene S., Gentile G., 2014, MNRAS, 441, 869

De Looze I. et al., 2014, A\&A, 571, A69

Dalla Vecchia C., Schaye J., 2012, MNRAS, 426, 140

Davé R., Thompson R., Hopkins P. F., 2016, MNRAS, 462, 3265

Davis M., Efstathiou G., Frenk C. S., White S. D. M., 1985, ApJ, 292, 371

Dehnen W., Aly H., 2012, MNRAS, 425, 1068

Disney M., Davies J., Phillipps S., 1989, MNRAS, 239, 939

Doi M. et al., 2010, AJ, 139, 1628

Draine B. T., Li A., 2007, ApJ, 657, 810

Draine B. T. et al., 2007, ApJ, 663, 866

Driver S. P., Popescu C. C., Tuffs R. J., Liske J., Graham A. W., Allen P. D., de Propris R., 2007, MNRAS, 379, 1022

Driver S. P. et al., 2009, Astron. Geophys., 50, 12

Driver S. P. et al., 2011, MNRAS, 413, 971

Durier F., Dalla Vecchia C., 2012, MNRAS, 419, 465

Dwek E., 1998, ApJ, 501, 643

Feldmann R., 2015, MNRAS, 449, 3274

Frenk C. S., White S. D. M., Davis M., Efstathiou G., 1988, ApJ, 327, 507

Furlong M. et al., 2015, MNRAS, 450, 4486

Furlong M. et al., 2017, MNRAS, 465, 722

Gadotti D. A., Baes M., Falony S., 2010, MNRAS, 403, 2053

Gilbank D. G., Baldry I. K., Balogh M. L., Glazebrook K., Bower R. G., 2010, MNRAS, 405, 2594

Gonzalez-Perez V., Lacey C. G., Baugh C. M., Lagos C. D. P., Helly J., Campbell D. J. R., Mitchell P. D., 2014, MNRAS, 439, 264

Groves B., Dopita M. A., Sutherland R. S., Kewley L. J., Fischera J., Leitherer C., Brandl B., van Breugel W., 2008, ApJS, 176, 438

Guidi G., Scannapieco C., Walcher C. J., 2015, MNRAS, 454, 2381

Gunawardhana M. L. P. et al., 2013, MNRAS, 433, 2764

Gunawardhana M. L. P. et al., 2015, MNRAS, 447, 875

Haardt F., Madau P., 2001, in Neumann D. M., Tran J. T. V., eds, Clusters of Galaxies and the High Redshift Universe Observed in X-rays

Hayward C. C., Smith D. J. B., 2015, MNRAS, 446, 1512

Hernández-Pérez F., Bruzual G., 2013, MNRAS, 431, 2612

Hewett P. C., Warren S. J., Leggett S. K., Hodgkin S. T., 2006, MNRAS, 367,454

Heyer M. H., Carpenter J. M., Snell R. L., 2001, ApJ, 551, 852

Hill D. T. et al., 2011, MNRAS, 412, 765

Hopkins P. F., 2013, MNRAS, 428, 2840

Hopkins A. M. et al., 2003, ApJ, 599, 971

Horiuchi S., Beacom J. F., Bothwell M. S., Thompson T. A., 2013, ApJ, 769,113

Hughes T. M. et al., 2015, A\&A, 575, A17

Hunt L. K., Hirashita H., 2009, A\&A, 507, 1327

James P. A. et al., 2004, A\&A, 414, 23

Jenkins A., Booth S., 2013, preprint (arXiv:1306.5771)

Jonsson P., 2006, MNRAS, 372, 2

Jonsson P., Groves B. A., Cox T. J., 2010, MNRAS, 403, 17

Kauffmann G. et al., 2003a, MNRAS, 341, 33

Kauffmann G. et al., 2003b, MNRAS, 341, 54

Kennicutt R. C., Jr, 1992, ApJ, 388, 310

Kennicutt R. C., Jr, 1998, ARA\&A, 36, 189

Kim J.-h. et al., 2014, ApJS, 210, 14

Kriek M., Conroy C., 2013, ApJ, 775, L16

Kroupa P., 2001, MNRAS, 322, 231

Lacey C., Cole S., 1994, MNRAS, 271, 676

Lagos C. D. P. et al., 2015, MNRAS, 452, 3815

Leitherer C. et al., 1999, ApJS, 123, 3

Li C., White S. D. M., 2009, MNRAS, 398, 2177 
Lupton R., Blanton M. R., Fekete G., Hogg D. W., O’Mullane W., Szalay A., Wherry N., 2004, PASP, 116, 133

Ly C., Malkan M. A., Kashikawa N., Ota K., Shimasaku K., Iye M., Currie T., 2012, ApJ, 747, L16

McAlpine S. et al., 2016, Astron. Comput., 15, 72

McKinnon R., Torrey P., Vogelsberger M., Hayward C. C., Marinacci F., 2017, MNRAS, 468, 1505

Maneewongvatana S., Mount D. M., 2001, On the Efficiency of Nearest Neighbour Searching with Data Clustered in Lower Dimensions. Springer-Verlag, Berlin

Maraston C., 2005, MNRAS, 362, 799

Mattsson L., De Cia A., Andersen A. C., Zafar T., 2014, MNRAS, 440, 1562

Mitchell P. D., Lacey C. G., Baugh C. M., Cole S., 2013, MNRAS, 435, 87

Murante G., Monaco P., Borgani S., Tornatore L., Dolag K., Goz D., 2015, MNRAS, 447, 178

Murphy E. J. et al., 2011, ApJ, 737, 67

Natale G., Popescu C. C., Tuffs R. J., Debattista V. P., Fischera J., Grootes M. W., 2015, MNRAS, 449, 243

Padmanabhan N. et al., 2008, ApJ, 674, 1217

Planck Collaboration XVI, 2014, A\&A, 571, A16

Popescu C. C., Tuffs R. J., 2002, MNRAS, 335, L41

Robotham A. et al., 2010, PASA, 27, 76

Rosas-Guevara Y. M. et al., 2015, MNRAS, 454, 1038

Saftly W., Camps P., Baes M., Gordon K. D., Vandewoude S., Rahimi A., Stalevski M., 2013, A\&A, 554, A10

Saftly W., Baes M., De Geyter G., Camps P., Renaud F., Guedes J., De Looze I., 2015, A\&A, 576, A31

Scannapieco C., Gadotti D. A., Jonsson P., White S. D. M., 2010, MNRAS, 407, L41

Scannapieco C. et al., 2012, MNRAS, 423, 1726

Schaller M., Dalla Vecchia C., Schaye J., Bower R. G., Theuns T., Crain R. A., Furlong M., McCarthy I. G., 2015, MNRAS, 454, 2277

Schawinski K. et al., 2014, MNRAS, 440, 889

Schaye J., 2004, ApJ, 609, 667

Schaye J., Dalla Vecchia C., 2008, MNRAS, 383, 1210

Schaye J. et al., 2010, MNRAS, 402, 1536

Schaye J. et al., 2015, MNRAS, 446, 521 (S15))

Schechter P., 1976, ApJ, 203, 297

Sharp R. et al., 2006, in McLean I. S., Iye M., eds, Proc. SPIE Conf. Ser. Vol. 6269, Ground-based and Airborne Instrumentation for Astronomy. SPIE, Bellingham, p. $62690 \mathrm{G}$

Sobral D., Smail I., Best P. N., Geach J. E., Matsuda Y., Stott J. P., Cirasuolo M., Kurk J., 2013, MNRAS, 428, 1128

Sodre L., Ribeiro da Silva A., Santos W. A., 2013, MNRAS, 434, 2503

Springel V., White S. D. M., Tormen G., Kauffmann G., 2001, MNRAS, 328,726

Springel V., Di Matteo T., Hernquist L., 2005, MNRAS, 361, 776

Stalevski M., Fritz J., Baes M., Nakos T., Popović L. Č., 2012, MNRAS, 420, 2756

Stalevski M., Ricci C., Ueda Y., Lira P., Fritz J., Baes M., 2016, MNRAS, 458,2288

Steinacker J., Baes M., Gordon K. D., 2013, ARA\&A, 51, 63

Stoughton C. et al., 2002, AJ, 123, 485

Strauss M. A. et al., 2002, AJ, 124, 1810

Taylor E. N. et al., 2011, MNRAS, 418, 1587

Taylor E. N. et al., 2015, MNRAS, 446, 2144

Torrey P. et al., 2015, MNRAS, 447, 2753

Trayford J. W. et al., 2015, MNRAS, 452, 2879 (T15)

Trayford J. W., Theuns T., Bower R. G., Crain R. A., Lagos C. d. P., Schaller M., Schaye J., 2016, MNRAS, 460, 3925

Tremonti C. A. et al., 2004, ApJ, 613, 898

Tuffs R. J., Popescu C. C., Völk H. J., Kylafis N. D., Dopita M. A., 2004 A\&A, 419, 821

Viaene S. et al., 2016, A\&A, 586, A13

Viaene S. et al., 2017, A\&A, 599, A64

Vogelsberger M. et al., 2014, MNRAS, 444, 1518

Wendland H., 1995, Adv. Comput. Math., 4, 389
White S. D. M., Frenk C. S., 1991, ApJ, 379, 52

White S. D. M., Rees M. J., 1978, MNRAS, 183, 341

Whitney B. A., 2011, Bull. Astron. Soc. India, 39, 101

Wiersma R. P. C., Schaye J., Smith B. D., 2009a, MNRAS, 393, 99

Wiersma R. P. C., Schaye J., Theuns T., Dalla Vecchia C., Tornatore L., 2009b, MNRAS, 399, 574

Wild V., Charlot S., Brinchmann J., Heckman T., Vince O., Pacifici C., Chevallard J., 2011, MNRAS, 417, 1760

Williams R. J., Quadri R. F., Franx M., van Dokkum P., Labbé I., 2009, ApJ, 691,1879

Witt A. N., Thronson H. A., Jr, Capuano J. M., Jr, 1992, ApJ, 393, 611

Xilouris E. M., Byun Y. I., Kylafis N. D., Paleologou E. V., Papamastorakis J., 1999, A\&A, 344, 868

York D. G. et al., 2000, AJ, 120, 1579

Zafar T., Watson D., 2013, A\&A, 560, A26

Zahid H. J., Yates R. M., Kewley L. J., Kudritzki R. P., 2013, ApJ, 763, 92

Zubko V., Dwek E., Arendt R. G., 2004, ApJS, 152, 211

Zurita A., Rozas M., Beckman J. E., 2000, A\&A, 363, 9

\section{APPENDIX A: SMOOTHING LENGTHS}

Star and gas particles in EAGLE function as tracers of the baryonic mass. Because particles are the smallest resolution elements in the simulations, the distribution of the material represented by a single particle is unresolved. However, some 3D form for the traced material needs to be assumed to facilitate radiative transfer with SKIRT. A kernel distribution is thus used to set the density profile of the stars and gas. A truncated Gaussian distribution is used to approximate the cubic spline kernel used by the EAGLE simulations in SKIRT (Altay \& Theuns 2013; Baes \& Camps 2015). As this is isotropic, it is parametrized solely by a position and a smoothing length.

Smoothing lengths are tracked by EAGLE for baryonic particles on the fly (see S15, Appendix A). These values are derived using the distance to the weighted $N$ th nearest neighbouring gas particle, to facilitate SPH interaction between gas and chemical enrichment of gas by stars. As such this kernel size represents the spatial smoothing of gaseous material well, but implies that for star particles the smoothing is entirely dependent on their proximity to gas.

For resolved disc galaxies in EAGLE this stellar smoothing is reasonable as the galaxies have high gas fractions, with star and gas particles being well mixed. However for a minority of gas-poor elliptical galaxies, the smoothing values may become extremely large (up to $\sim 70 \mathrm{kpc}$ ). This distorts the surface brightness profiles to become kernel shaped, and renders them much more extended than the actual stellar surface density.

To alleviate this problem, we re-compute more appropriate smoothing lengths for EAGLE star particles within each galaxy. There is no unique smoothing scale for star particles that can be defined, as they do not interact with each other using an SPH kernel. Using the same smoothing length calculation between star particles as between gas particles also results in significantly smaller smoothing lengths, due to a higher fraction of galaxy mass being in stars than gas, and such small smoothing lengths yields unrealistic granularity in galaxy images. For this reason we use a somewhat ad hoc method of 'morphological convergence', observing galaxy images smoothed on a variety of scales, in a similar vein to Torrey et al. (2015).

We use a $k d$-tree algorithm (Maneewongvatana $\&$ Mount 2001) to identify nearby star particles. This is performed for each galaxy as it is extracted from the simulation data. We find that using as a smoothing length the distance to the 64th nearest neighbouring star 

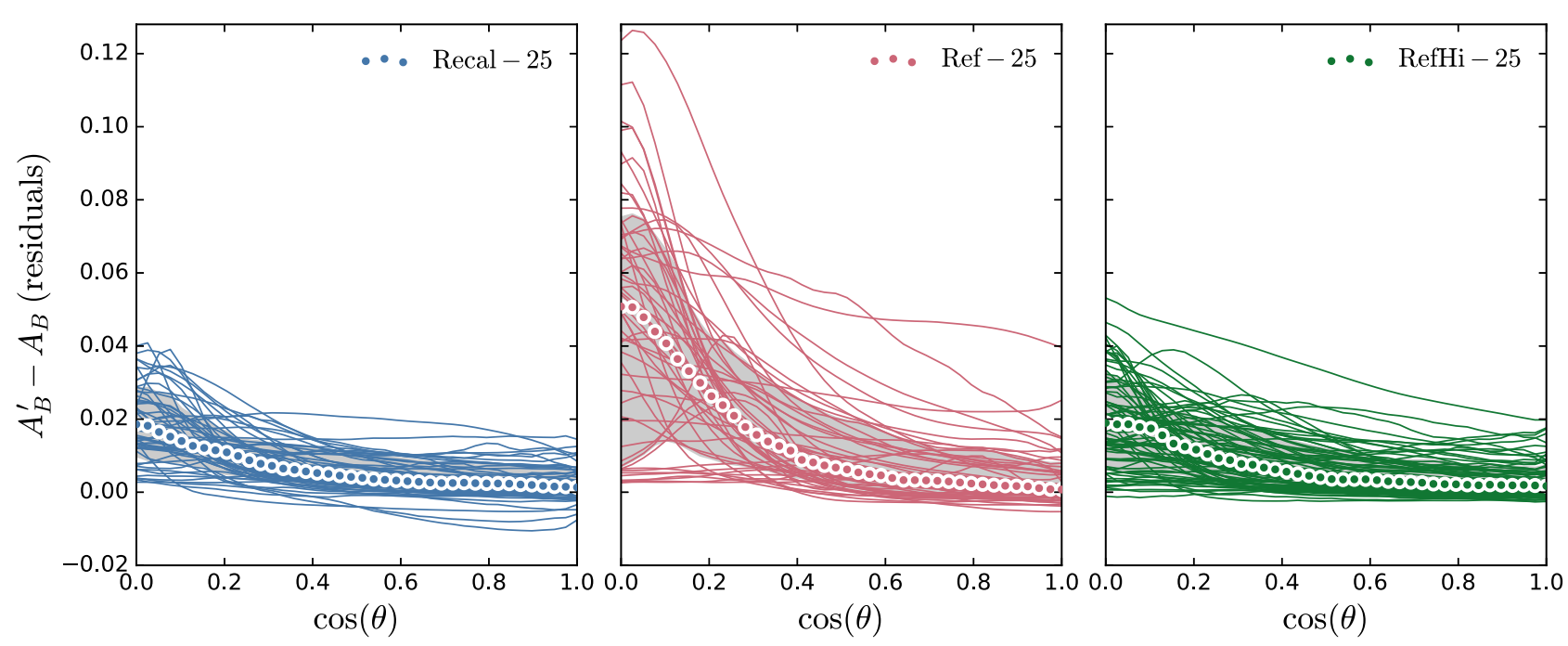

Figure A1. The difference between $r$-band attenuation in EAGLE galaxies when using no smoothing $\left(A_{r}^{\prime}\right)$ and employing the standard nearest neighbours smoothing used in this work $\left(A_{r}\right)$ plotted as a function of inclination, $\cos (\theta)$. The panels from left to right represent simulations Recal-25, Ref-25 and RefHi-25, and are also coloured blue red and green, respectively. Thin Lines represent $A_{r}^{\prime}-A_{r}$ values of individual galaxies taken from their respective simulations at 40 inclinations evenly spaced in $\cos (\theta)$. Shaded circles represent the median $A_{r}^{\prime}-A_{r}$ value of galaxies at each $\cos (\theta)$ value, with grey shading illustrating the 16th-84th percentile range. We see that not using stellar smoothing leads to a slightly stronger inclination dependence for orientation, with increased attenuation for edge-on galaxies. The maximal difference is seen for edge-on galaxies in the Ref- 25 simulation, $\Delta A_{B} \approx 0.1$, with typical values for edge-on galaxies of $\Delta A_{B} \approx 0.05$.

particle works well, in the sense that this yields reasonable galaxy images, avoiding both unrealistic granularity and oversmoothing. Using the re-computed or simulation smoothing lengths make only marginal difference to the scientific results presented in this work. Intrinsic properties (i.e. without dust effects) are of course unaffected, as all light emitted by material within the $30 \mathrm{pkpc}$ aperture is measured for consistency with T15. The effect on dust attenuated properties is small because the smoothing lengths differ most in large galaxies where there is minimal gas and thus minimal attenuation.

To constrain the effect of stellar smoothing on the attenuation measured for EAGLE galaxies, we compare attenuation measured for EAGLE galaxies without any smoothing of sources (i.e. treating star particles as point sources) to those measured using the re-computed smoothing lengths. Fig. A1 is set out in a similar way to Fig. 4, except we plot the difference between the $r$-band attenuation without smoothing $\left(A_{r}^{\prime}\right)$ and with smoothing $\left(A_{r}\right)$ on the $y$-axis. The thin coloured lines show the residuals for individual galaxies at different orientations, and the data points show the median residuals. Again, all galaxies of mass $M_{\star}>10^{10} \mathrm{M}_{\odot}$ are included for each of the Ref-25 Recal-25 and RefHi-25 simulations.

We see that, in general, treating stellar particles as point sources leads to a stronger dependence of attenuation on inclination, with more attenuation for edge on inclinations. This can be understood as a higher fraction of the stellar emission emanating from near the disc plane when no smoothing is applied, as the smoothing effectively thickens the emissivity distribution of the stellar disc. Although this effect is measurable, the difference is $\lesssim 10$ per cent for the galaxies in this sample, suggesting that even an extreme choice in stellar smoothing has only a marginal effect on the integrated dust reddening for these galaxies. The difference is most pronounced for the low resolution Ref-25 galaxies, because this has lower stellar particle resolution and larger smoothing lengths for the same mass range of $M_{\star}>10^{10} \mathrm{M}_{\odot}$.

\section{APPENDIX B: RESOLUTION AND CONVERGENCE}

\section{B1 Radiative transfer}

Here, we test the convergence of photometry results respect to the sampling of photon packages. We look at both the spectral resolution used to represent SEDs, and the number of photon packets sampled at each wavelength. In order to test this efficiently for our fiducial model, we randomly select a test sample of galaxies in nine contiguous and logarithmically spaced mass bins, over the range $9<\log _{10}\left(M_{\star} / \mathrm{M}_{\odot}\right) \leq 11.6 .200$ galaxies are randomly selected from each mass bin, except in the two highest mass bins where all the galaxies are sampled due to insufficient counts. The highest and second highest bins contain 23 and 64 galaxies, respectively.

\section{B1.1 Sampling noise}

SKIRT tracks an equal number, $N_{\gamma}$, of photon packets per wavelength bin. The stochastic emission and absorption of these photon packets introduces Poisson noise into the photometric measurements. The degree of this variation depends not only on $N_{\lambda}$, but also on the emissivity at each wavelength, and the complex distribution of sources and dust in the galaxy. A natural target level of convergence is variation comparable to photometric errors in SDSS observations, of the order of $\sim 0.01 \mathrm{mag}$ for griz and $\sim 0.02 \mathrm{mag}$ for the $u$ band, dominated by uncertainty in the un-modelled atmospheric effects at Apache Point (Padmanabhan et al. 2008).

We test photometric convergence by running separate SKIRT simulations launching $N_{\lambda}=2^{11}, 2^{13}$ and $2^{15}$ photon packets per wavelength bin on each of our test sample galaxies. We then compare the variation in ugrizYJHK photometry between the $N_{\lambda}=2^{15}$ run and the lower $N_{\lambda}$ runs. Fig. B1 shows this level of variation. Both the median and maximum variations are below 0.01 mag for griz 

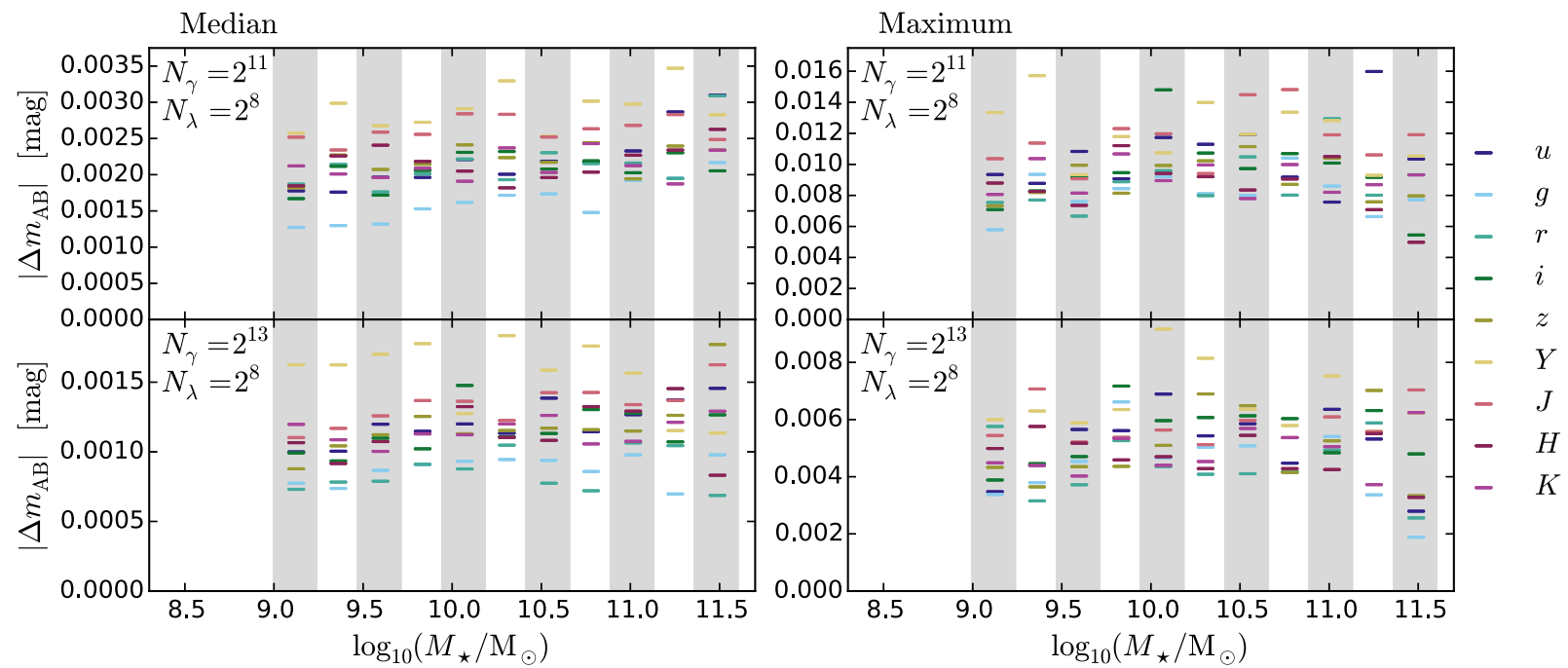

Figure B1. SDSS ugriz and UKIRT YJHK absolute magnitude convergence properties for sample EAGLE galaxies (Section B) varying the number of photon packets launched per wavelength bin, $N_{\gamma}$, at a constant spectral resolution. SKIRT simulations with low, medium and high $N_{\gamma}$ values of $2^{11}, 2^{13}$ and $2^{15}$, respectively, are run for each galaxy. The top and bottom panels of each column then show the comparison of the low and medium $N_{\gamma}$ simulations with the high value, respectively. The left-hand panel shows the median absolute magnitude difference in each bin for the nine photometric bands, the right-hand panel shows the maximum difference.
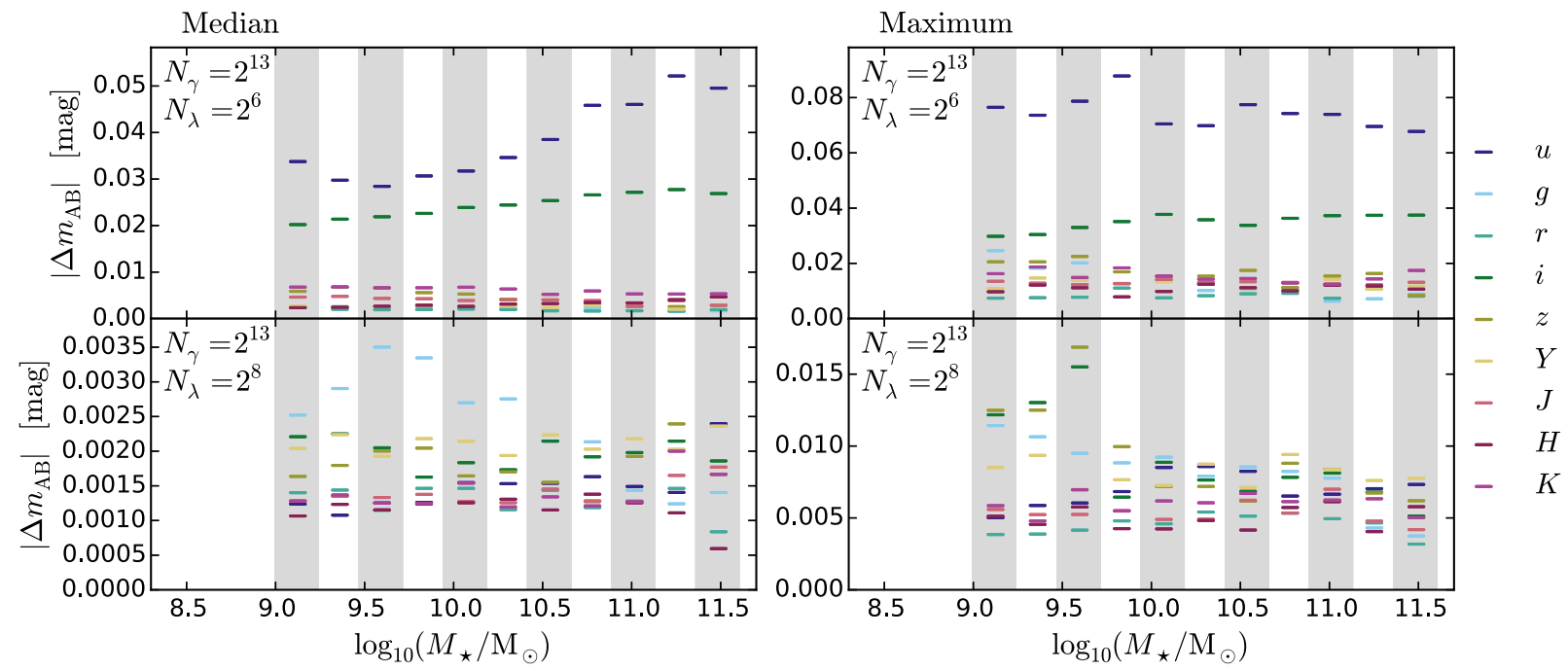

Figure B2. As Fig. B1, but now exploring convergence as a function of continuum spectral resolution, $N_{\lambda}$, at fixed $N_{\gamma}$. SKIRT simulations with low, medium and high $N_{\lambda}$ values of $2^{7}, 2^{8}$ and $2^{9}$, respectively, are run for each galaxy. Again, the low and medium resolutions are compared to the high resolution in the top and bottom panels of each column, respectively.

and 0.02 mag for the $u$ band for $N_{\gamma}=2^{13}$, the number we used in the paper.

\section{B1.2 Spectral resolution}

To sample SEDs, SKIRT performs radiative transfer for a grid of wavelengths. The number of wavelengths we choose is a trade-off between spectral resolution and computational expense. For our purposes, we want to resolve the continuum well enough to capture the overall shape of the SED and produce accurate photometry in arbitrary optical broad-bands, as well as focusing on certain spectral indices of interest. After some initial experimentation, we begin with a superposed grid of wavelengths:

(i) $2^{8}$ (256) logarithmically spaced wavelengths to sample the continuum between 280 and $2500 \mathrm{~nm}$. (ii) 33 wavelengths to sample the peak and continuum either side of 11 prominent spectral lines in emission line galaxies (Stoughton et al. 2002)

(iii) 22 evenly spaced wavelengths to better sample the $\mathrm{H} \alpha$ and O II line profiles.

(iv) 10 logarithmically spaced wavelengths from 150 to $280 \mathrm{~nm}$ to sample the UV slope.

(v) 12 additional wavelengths about the $4000 \AA$ break.

We test numerical convergence by measuring the variation between individual galaxies when different spectral resolutions are used. We vary the continuum wavelength grid resolution, (i), using the standard value of $2^{8}$ as medium resolution and $2^{7}$ and $2^{9}$ as low and high resolution respectively and comparing the standard and low resolutions to the high resolution in each plot. Fig. B2 shows this level of variation. 


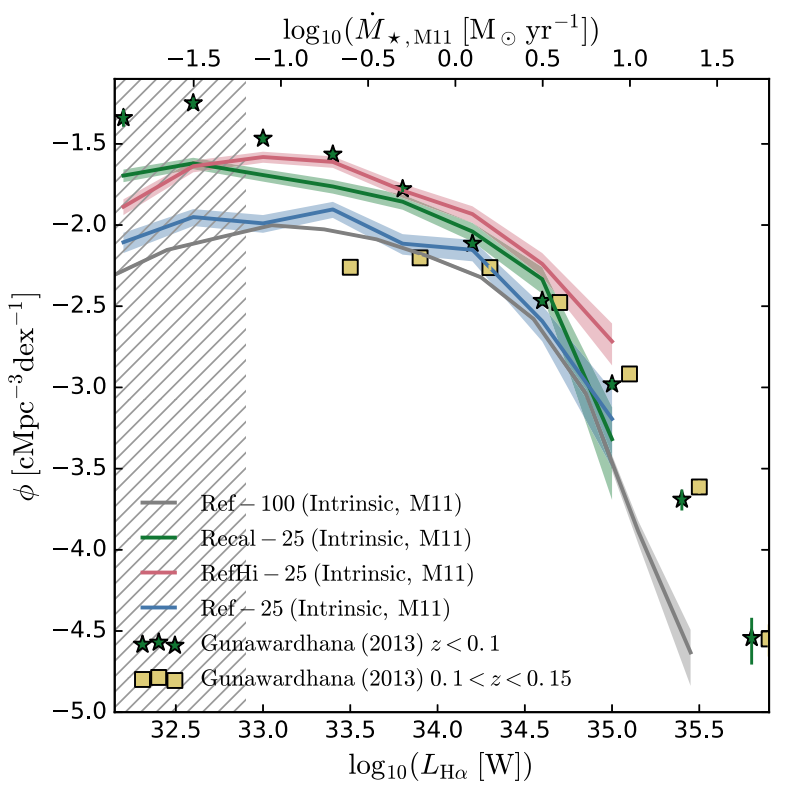

Figure B3. As Fig. 12, but excluding $\mathrm{C} 15$ recalibrated lines and including the Ref-25 and RefHi-25 H $\alpha$ luminosity functions. The Ref-100, Recal-25, RefHi-25 and Ref-25 luminosity functions are represented as grey, green, red and blue lines, respectively, with Poisson error indicated by the shaded regions of the same colour.

We find that the median variation is $<0.01$ mag for all bands at standard resolution, $N_{\lambda}=2^{8}$. When looking at the most extreme outliers in each bin we see that the most extreme differences are $\sim 0.015$ mag in the lowest mass bin. We decide this to be sufficient resolution for our purposes.

\section{B2 Covergence of $\mathrm{H} \alpha$ luminosity and flux}

Here we investigate the convergence properties of the $\mathrm{H} \alpha$ line fluxes, by comparing between all the simulations listed in Table 1 in Fig. B3. Comparing Ref-100 and Ref-25 simulations, plotted as grey and blue lines, respectively, isolates the effects of volume because the subgrid calibration and resolution are the same. We see that the Ref-25 LF agrees better with Ref-100 than any of the other $25^{3} \mathrm{Mpc}^{3}$ boxes. However the Ref-25 LF is still between 0 and 0.3 dex higher at all luminosities sampled. The higher number density of $\mathrm{H} \alpha$-emitting galaxies is likely due to a $25^{3} \mathrm{Mpc}^{3}$ being too small to represent large-scale modes in the density distribution, and thus does not sample massive haloes. As was shown in Trayford et al. (2016), star formation is significantly suppressed within these environments in the EAGLE simulation. This could lead to the lower normalization of the LF in the Ref-100.

Comparing the Ref-25 and Recal-25 LFs instead tests 'weak' convergence (defined by S15) with resolution. The Recal-25 LF is in general higher still, typically by $\sim 0.2 \mathrm{dex}$. The higher normalization of the Recal-25 LF is attributable to the effect of resolution on passive fractions of galaxies. As was discussed in T15, coarse sampling of feedback events paired with the resolution of the starforming component of galaxies contributes to the surplus of passive galaxies seen at $\left(M_{\star} / \mathrm{M}_{\odot}\right) \lesssim 10$ in the lower resolution simulations. Because these resolution effects become significant at a factor of $\sim 8$ lower mass at higher resolution, the proportion of star-forming galaxies at relatively low mass is more realistic. This contributes to boosting the Recal-25 LF closer to observations.

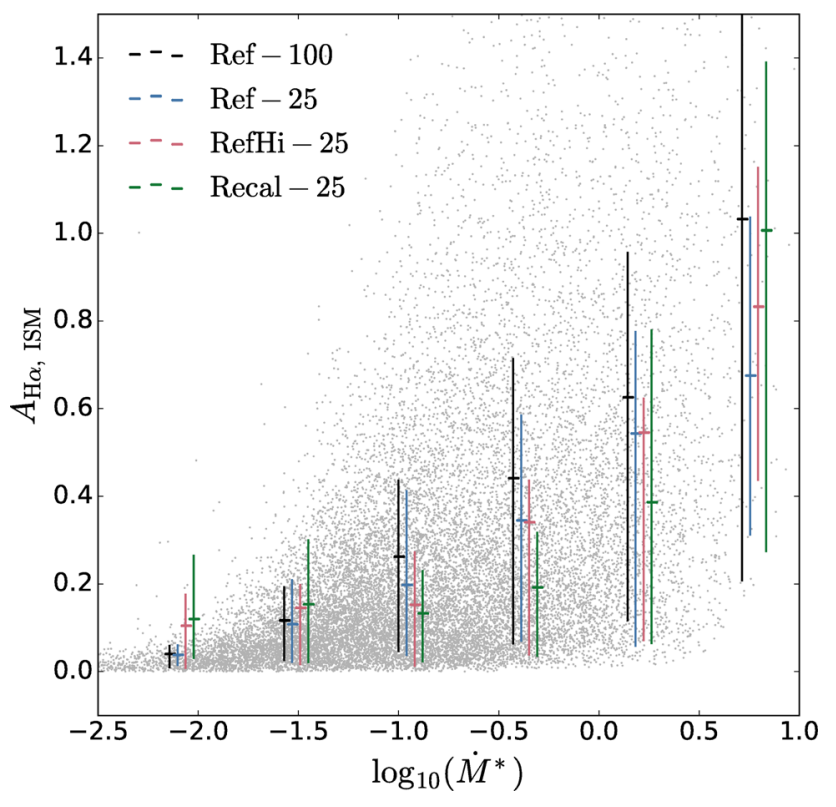

Figure B4. Plot of $A(\mathrm{H} \alpha$, ISM) (equation $\mathrm{B} 1)$ as a function of star formation. Individual galaxies with $\log _{10}\left(M_{\star} / \mathrm{M}_{\odot}\right)>8.5$ from each simulation of Table 1 are plotted as grey points. Average values for each simulation are overplotted as coloured points, with error bars indicating the 16th-84th percentile ranges. We see that the attenuation generally increases as a function of star formation rate, exhibiting large scatter skewed towards high values. The average relations agree between simulations within errors, suggesting that $A(\mathrm{H} \alpha, \mathrm{ISM})$ is well converged in our modelling.

We also investigate the attenuation of the $\mathrm{H} \alpha$ line by the diffuse dust component, $A(\mathrm{H} \alpha$, ISM), using the simulations listed in Table 1 in Fig. B3. We look specifically at the ISM attenuation here, as this is controlled by the ISM structure that may vary with resolution. Taking

$A(\mathrm{H} \alpha, \mathrm{ISM})=2.5 \log _{10}\left(L_{\mathrm{H} \alpha, \text { SKIRT }} / L_{\mathrm{H} \alpha, \text { intrinsic }}\right)$

we plot $A(\mathrm{H} \alpha$, ISM $)$ as a function of star formation rate for individual galaxies with $\log _{10}\left(M_{\star} / \mathrm{M}_{\odot}\right)>8.5$ from each simulation as grey points, overplotting the average attenuation in bins of star formation rate for each simulation in different colours, and indicating the scatter using the 16 th- 84 th percentile ranges. We see that attenuation generally increases with star formation rate but exhibits a large scatter, skewed to high values. This is consistent with general observational trends (e.g. Gunawardhana et al. 2015). We notice that the typical attenuation values are similar for all the simulations, and consistent with each other within the scatter, suggesting $A(\mathrm{H} \alpha)$ converges with resolution. This is consistent with what we find for Fig. B4, that attenuation is limited by the artificial pressurization of the EAGLE ISM rather than resolution, and attenuation would likely increase if gas were able to cool to lower temperatures.

\section{APPENDIX C: FITTING SKIRT RESULTS USING THE T15 MODEL}

To make a detailed comparison between the radiative transfer photometry presented in this work and that of $\mathrm{T} 15$, we calibrate the dustscreen parameters used by T15 to the results obtained with SKIRT. Comparing these parameters to their fiducial T15 values helps us understand how the models differ. This can be achieved by finding the ML parameter values using an MCMC approach. 
The flux density for an EAGLE galaxy measured in a certain band using the T15 model, $F_{\mathrm{T} 15}$, can be expressed as

$F_{\mathrm{T} 15}=\left(F_{\mathrm{o}}+F_{\mathrm{y}} \mathrm{e}^{-\hat{\tau}_{\mathrm{BC}}}\right) \mathrm{e}^{-\hat{\imath}_{\mathrm{ISM}} O(\theta \mid q)}$

where

$\hat{\tau}_{\mathrm{ISM}}=\tau_{\mathrm{ISM}} f_{\mathrm{M}} f_{\mathrm{Z}}\left(\frac{\lambda_{\text {eff }}}{550 \mathrm{~nm}}\right)^{-0.7}$,

$\hat{\tau}_{\mathrm{BC}}=\tau_{\mathrm{BC}} f_{\mathrm{M}} f_{\mathrm{Z}}\left(\frac{\lambda_{\text {eff }}}{550 \mathrm{~nm}}\right)^{-0.7}$.

Here, $f_{\mathrm{M}}$ and $f_{\mathrm{Z}}$ are the star-forming gas mass and metallicity in units of the Milky Way value, respectively, $\lambda_{\text {eff }}$ is the effective wavelength of the filter, and $F_{\mathrm{o}}$ and $F_{\mathrm{y}}$ are the intrinsic flux densities for star particles older and younger than $10 \mathrm{Myr}$, respectively. The $O(\theta \mid q)$ term accounts for galaxy orientation, as detailed in section 3.2.3 of T15. The free parameters of the T15 model which we fit for, are $\tau_{\mathrm{ISM}}, \tau_{\mathrm{BC}}$ and $q$, representing the typical ISM optical depth, birth cloud optical depth and dust disc axial ratio.

The attenuation applied in T15 is deterministic, apart from the randomized orientation term, $O(\theta \mid q)$, where the disc inclination angle is sampled uniformly in $\cos (\theta)$. However, as we store the inclination angle of each SKIRT image, the expected $F_{\mathrm{T} 15}$ value corresponding to a particular SKIRT galaxy observation, $F_{\mathrm{S}}$, is fully deterministic.

Clearly, no parametrization of the simple T15 model can provide perfect agreement with the SKIRT photometry. The inclination also has an associated uncertainty. So that any possible $F_{\mathrm{S}}$ has a finite likelihood of being observed with a particular T15 parametrization, we build in a Gaussian observational tolerance. This contributes to the log-likelihood as

$\ln \mathcal{L}\left(F \mid F_{\mathrm{T} 15}, \sigma\right)=C-\frac{1}{2 \sigma^{2}} \sum_{i=1}^{n}\left(F_{i}-F_{\mathrm{T} 15, i}\right)^{2}$,

where $C$ is a constant, $\sigma$ is a fixed uncertainty, and we sum over each randomly oriented ugrizYJHK observation of an EAGLE galaxy sample. The constant $\sigma$ value means that better-resolved galaxies generally provide stronger constraints on the model, so the likelihood is effectively luminosity weighted. The exact value of $\sigma$ we use is unimportant as we aim to maximize $l$, but should be comparable to the observations to avoid numerical errors. We use the fifth percentile of all $F_{\mathrm{S}}$ values as $\sigma$. For simplicity, we do not explicitly incorporate an additional uncertainty on our input inclination angles.

With an imperfect model, the galaxies we use to constrain our fit will affect the recovered ML parameters. However, using all galaxies may not provide the best parametrization for those where dust is effective. For insufficiently resolved galaxies, the SKIRT dust modelling is itself unreliable, and dust effects are anyway minimal. For the most luminous galaxies the simple geometric assumptions of T15 are inappropriate and do not help constrain the parameters. For this reason, we select a sample in stellar mass. To capture sufficiently resolved galaxies over which the disc geometry assumption is appropriate, we select galaxies in the range $10^{9.75}<M_{\star} / \mathrm{M}_{\odot}<10^{11}$.

Initially we assume uniform prior distributions for each parameter and fit simultaneously, with the conservative ranges $q \in[0$, $1), \tau_{\mathrm{BC}} \in[0,10)$ and $\tau_{\mathrm{ISM}} \in[0,10)$. However, we found that the $\tau_{\mathrm{BC}}$ parameter tends to $\sim 10$. Indeed, relaxing the prior to $\tau_{\mathrm{BC}} \in[0$, 1000 ) yields a median $\sim 800$, such that $F_{\text {y }}$ contributes effectively nothing to $F_{\mathrm{T} 15}$. The reason for this may be that the spectra representing stellar populations younger than $10 \mathrm{Myr}$ are intrinsically different in the models, and the clearing time-scale for birth clouds
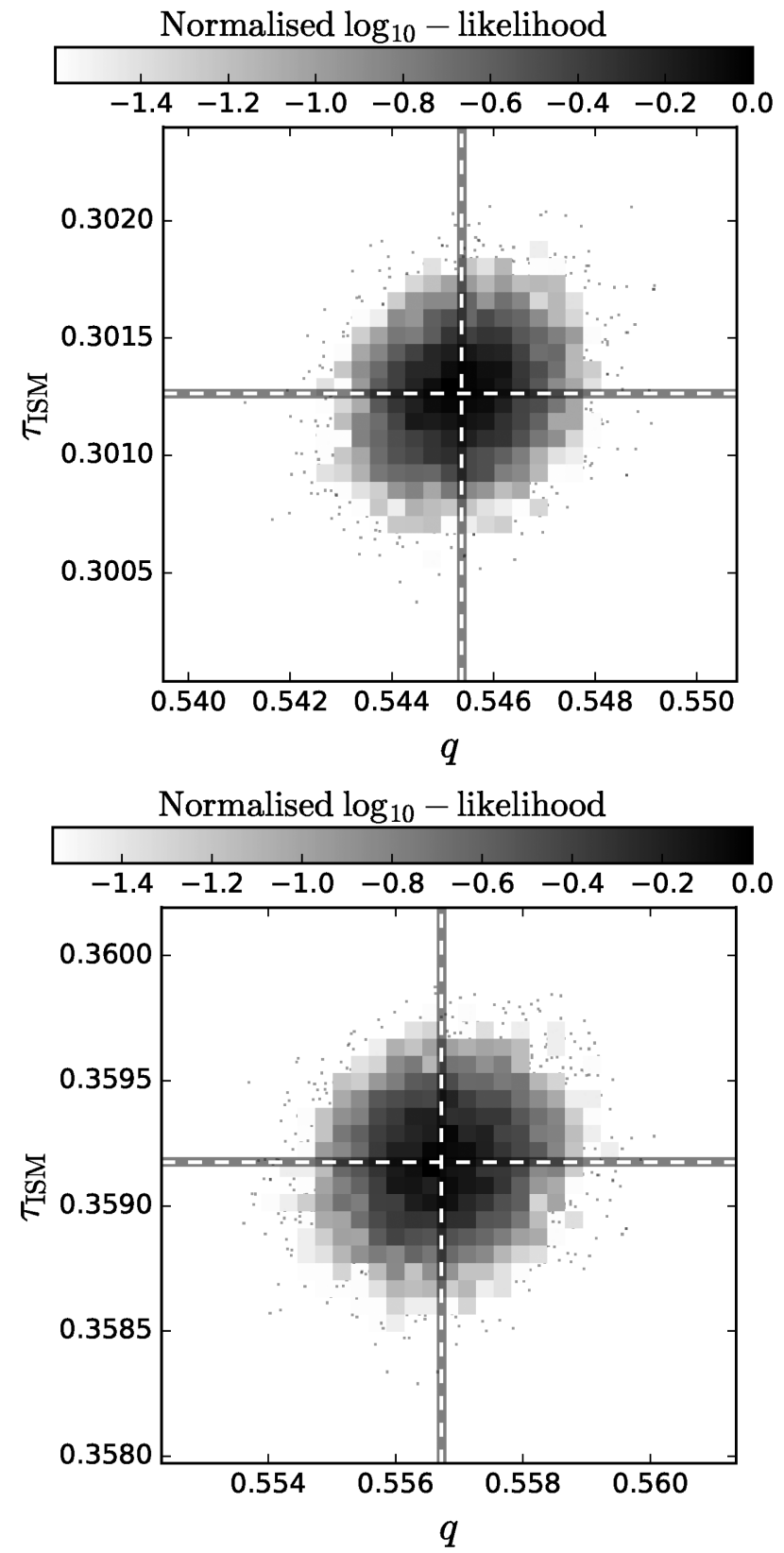

Figure C1. Shaded maps of likelihood for T15 $\tau_{\text {ISM }}$ and $q$ values fitting the SKIRT photometry of galaxies from the Ref- 100 simulation. The $\tau_{\mathrm{BC}}$ parameter is fixed to be $2 \tau_{\mathrm{ISM}}$. Where the normalized $\log _{10}$-likelihood falls below -1.5 , the individual Markov-chain samplings are plotted. The top panel represents the fiducial SKIRT model, while the bottom panel represents the 'uncalibrated' SKIRT model discussed in Appendix D. These are generated by constructing the posterior distribution using an MCMC method, as described in Section 5.2. The white dotted lines indicate the median value for each parameter. The ML parameter values are taken from the three dimensional distribution in parameter space, and listed in table 3.

is longer in Charlot \& Fall (2000) (30 Myr). While T15 uses the GALAXEV spectra (Bruzual \& Charlot 2003), the SKIRT model uses the MAPPINGS-III spectra (Groves et al. 2008). While both models account for birth cloud reddening, the MAPPINGS-III spectra include emission lines and a different ionizing spectrum (from Leitherer et al. 1999) than GALAXEV.

As a second approach, we try fixing $\tau_{\mathrm{BC}}=2 \tau_{\mathrm{ISM}}$, as suggested in the fiducial Charlot \& Fall (2000) model and T15, while assuming 


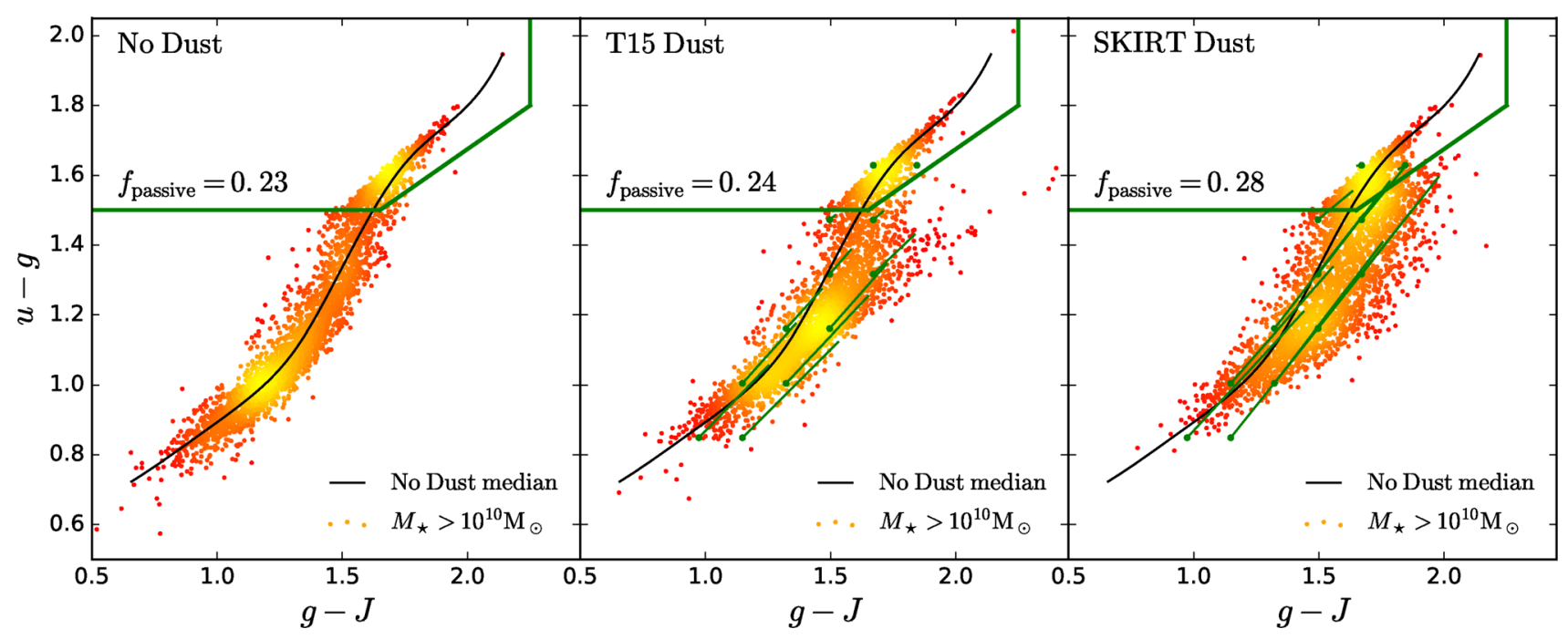

Figure D1. As Fig. 9, except using the uncalibrated SKIRT model with values of $f_{\text {dust }}=0.4$ and $f_{\text {PDR }}=0.2$. We see that this model marginally improves agreement with the observed blue and red fractions for masses $M_{\star}>10^{10} \mathrm{M}_{\odot}$.

the same priors for $\tau_{\mathrm{ISM}}$ and $q$. The $\tau_{\mathrm{BC}}$ parameter has only marginal influence on the photometry, as it only affects a small fraction of stars, and the T15 value of $\sim 0.67$ already significantly reduces their contribution. We therefore expect that fixing $\tau_{\mathrm{BC}}=2 \tau_{\mathrm{ISM}}$ rather than allowing it to freely vary over the range $[0,1000)$ has minimal influence on the other parameters, such that all parameter values remain physically plausible. We find that including this constraint yields ML $q$ and $\tau_{\text {ISM }}$ values only $\sim 8$ percent and $\sim 11$ percent higher, respectively. We therefore use this second approach as our default procedure.

With this set-up we recover ML values for the three parameters, which are encouragingly very close to the values assumed in T15, as discussed in Sections 5.2 and 7. Fig. C1 shows the posterior distribution constructed for the T15 model parameters given the SKIRT data, with the ML listed in Table 3 . We use $1 \times 10^{5}$ samples, employing a burn-in of $1 \times 10^{4}$ samples and a thinning factor of 5. The results of this are presented in Table 3, and discussed in Section 5.2. The top panel of Fig. $\mathrm{C} 1$ shows the constructed posterior distribution.

\section{APPENDIX D: COMPARING FIDUCIAL AND UNCALIBRATED SKIRT MODELS}

The $f_{\text {dust }}$ and $f_{\mathrm{PDR}}$ parameters are the two values in our dust model that are not assigned using the simulation output. These effectively scale the optical depths in the diffuse and birth cloud components, respectively. The parameters of our fiducial SKIRT model were calibrated using local dust scaling relations, indicative of dust mass and temperature, presented in the companion study of Camps et al. (2016, C16). This is desirable as it allows the same model to be consistent with observations over a large spectral range, from optical to FIR wavelengths. FIR measurements also provide a more direct measurement of dust mass than optical attenuation, which depends strongly on star formation histories and the geometries of stellar and ISM distributions. The C16 calibrated values used in our fidu- cial model are $f_{\text {dust }}=0.3$ and $f_{\mathrm{PDR}}=0.1$. Without FIR constraints, we would default to the best literature values for our assumed dust parameters.

In order to test the effect our choice of dust parameters has on our results, we also generated results using literature values for the parameters of $f_{\text {dust }}=0.4$ (Draine \& Li 2007) and $f_{\mathrm{PDR}}=0.2$ (Groves et al. 2008), hereby referred to as the 'uncalibrated' SKIRT model. For comparison, we apply the ML fitting procedure presented in Appendix $\mathrm{C}$ to the uncalibrated model and plot the posterior distribution as the bottom panel in Fig. C1.

Comparing the posterior distributions of the fiducial and uncalibrated SKIRT models reveals that the ML values for $\tau_{\text {ISM }}$ are $\sim 10$ per cent below and above their T15 values, respectively. The $q$ values for both models are $\sim 0.4$ dex higher than T15, with the uncalibrated model giving a $q$ value $\sim 10$ per cent higher than fiducial. ${ }^{12}$ Overall, a similar level of agreement with T15 is achieved for both the uncalibrated and fiducial SKIRT models.

To exhibit the effects of the calibration on the overall photometry, we also plot $u g J$ colour-colour distributions in Fig. D1. This is the same plot as Fig. 9, except using the uncalibrated rather than fiducial SKIRT model. We see that the higher optical depth normalization has a small effect on the colours, shifting galaxies to marginally redder colour in both indices. It seems that the effects on passive fractions are relatively minor, with only a $\sim 7$ per cent change in the passive fractions compared to 9 .

\footnotetext{
${ }^{12}$ This comparison also highlights incompatibility between the screen and SKIRT models; the $q$ parameter that independently describes geometry in T15 has different ML values for two SKIRT models with identical geometries but re-scaled dust optical depths. However the dependence of $q$ on optical depth is relatively weak. The change in $\tau_{\text {ISM }}$ is $\sim 10$ times larger in terms of the marginalized parameter uncertainty.
}

This paper has been typeset from a $\mathrm{T}_{\mathrm{E}} \mathrm{X} / \mathrm{L} \mathrm{T} \mathrm{T}_{\mathrm{E}} \mathrm{X}$ file prepared by the author. 